\title{
Nonlinear Periodic Adaptive Control for Linear Time-Varying Plants
}

\author{
by \\ Volodymyr Rudko
}

\author{
A thesis \\ presented to the University of Waterloo \\ in fulfillment of the \\ thesis requirement for the degree of \\ Master of Applied Science \\ in \\ Electrical and Computer Engineering
}

Waterloo, Ontario, Canada, 2013

(C) Volodymyr Rudko 2013 
I hereby declare that I am the sole author of this thesis. This is a true copy of the thesis, including any required final revisions, as accepted by my examiners.

I understand that my thesis may be made electronically available to the public. 


\begin{abstract}
In adaptive control the goal is to deal with systems that have unknown and/or timevarying parameters. Adaptive control techniques have been developed since 1950's and most results were proven in the cases when the time-variations were non-existent or slow. However the results pertaining to systems with fast time-variations are still limited, in particular, when it comes to plants with unstable zero dynamics.

In this work we adopt the controller design technique from the area of gain scheduling, where the time-varying parameter is assumed to be measurable. We propose the design of a nonlinear periodic controller, where in each period the state and parameter values are estimated and an appropriate stabilizing control signal is applied. It is shown that the closed loop system is stable under fast parameter variations with persistent jumps: the trajectory of the closed loop state in response to the initial condition is bounded by a decaying exponential plus a gain times the size of the noise. Our approach imposes several constraints on the plant; however, we show that there exists at least one interesting class of systems, which includes plants with unstable zero dynamics, that can be stabilized by our controller.
\end{abstract}




\section{Acknowledgements}

I would like to thank my research supervisor, Professor Daniel Miller, who oversaw my work and provided a lot of help with choosing the research direction and solving the problems I encountered along the way. I am also grateful to Darrell Gaudette who gave me advice pertaining to simulations. 


\section{Dedication}

This work is dedicated to the late Volodymyr Fedorovych Chaika who was my inspiration to pursue graduate studies. 


\section{Table of Contents}

$\begin{array}{lc}\text { List of Figures } & \text { viii }\end{array}$

1 Introduction $\quad 1$

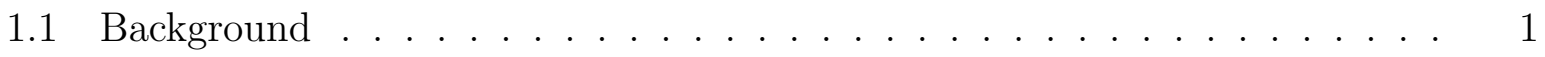

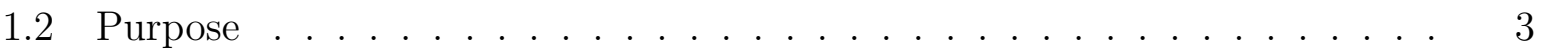

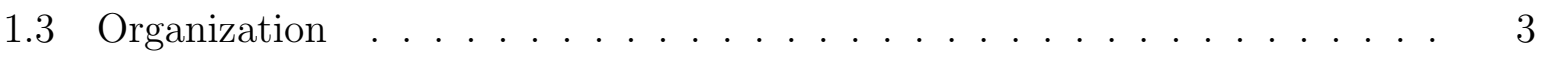

2 Mathematical Preliminaries $\quad 4$

3 Problem Formulation $\quad 6$

3.1 Adaptive Control of Time-Varying Systems . . . . . . . . . . . . . . 6

3.2 The Gain Scheduling Result . . . . . . . . . . . . . . . . 7

3.3 The Proposed Approach . . . . . . . . . . . . . . . . . 12

4 Noise Free Case $\quad 16$

4.1 State Estimation . . . . . . . . . . . . . . . . . 17

4.2 Parameter Estimation . . . . . . . . . . . . . . . 23

4.3 Discrete-Time Stability Analysis . . . . . . . . . . . . . . . . . 30

4.4 Intersample Behaviour . . . . . . . . . . . . . . . . . . 38

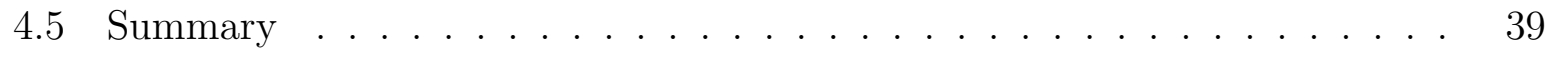


5 System in Presence of Noise $\quad 40$

5.1 State Estimation . . . . . . . . . . . . . . . . . . . . 42

5.2 Parameter Estimation _. . . . . . . . . . . . . . . 44

5.3 Discrete-Time Stability Analysis . . . . . . . . . . . . . . . . . . . . . 49

5.4 Handling Parameter Jumps . . . . . . . . . . . . . . . . . . . . 59

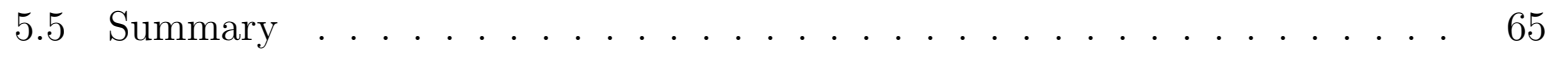

$\begin{array}{lll}6 & \text { Examples and Simulations } & 67\end{array}$

6.1 Controller Construction Algorithm . . . . . . . . . . . . . . . . . 67

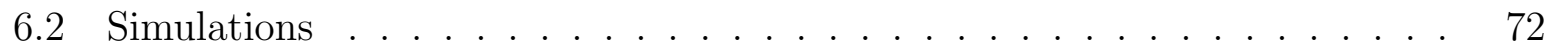

$\begin{array}{lll}7 & \text { Conclusions } & 78\end{array}$

$\begin{array}{lr}\text { APPENDICES } & 80\end{array}$

$\begin{array}{lr}\text { A Proofs } & 81\end{array}$

$\begin{array}{lr}\text { References } & 89\end{array}$ 


\section{List of Figures}

3.1 Closed loop system considered in this thesis . . . . . . . . . . . . 7

4.1 System setup when noise is not present . . . . . . . . . . . . . . 17

4.2 Control signal during $(k+1)$ 'th period . . . . . . . . . . . . . 18

5.1 System setup with noise injected at the input and output interfaces . . . . 41

6.1 Polygonal set . . . . . . . . . . . . . . . . . . 70

6.2 Example 1: output and control signals . . . . . . . . . . . . 73

6.3 Example 1: trajectories of the states and the parameter . . . . . . . 73

6.4 Example 2: discontinuous $u_{1}$ and $u_{2} \ldots \ldots$. . . . . . . . . . 75

6.5 Example 2: piecewise continuous $u_{1}$ and $u_{2}$ after interpolation . . . . . . 75

6.6 Example 2: output and control signals . . . . . . . . . . . . . . . 77

6.7 Example 2: trajectories of the states and the parameter . . . . . . . . . 77 


\section{Chapter 1}

\section{Introduction}

\section{$1.1 \quad$ Background}

In adaptive control the goal is to deal with systems that have unknown and/or timevarying parameters. A classical example of an adaptive controller is a linear time-invariant (LTI) compensator with adjustable parameters. A tuning mechanism is used to adjust the compensator's parameters to appropriately match the plant. The latter often causes the controller to become nonlinear.

Starting in the 1950's, adaptive control methods have been adopted in an attempt to control systems in which the parameters are uncertain and time-varying. However the solution to such problems could not be found and the focus shifted to a simplified scenario in which the system parameters are fixed, but unknown. Even the simplified problem turned out to be a challenge and a generalized solution could not be found until the early 1980s: [10], [25], [29]. The developed approaches often yielded poor transient responses and loss of stability in the face of unmodeled dynamic and bounded disturbances [30]. A number of methods have been developed to resolve these issues, which include the Certainty Equivalence approach (e.g. see [11], [16] and the contained references), the prerouted logic based switching approach (e.g. see [7] and [22]) as well as more sophisticated approaches such as supervisory and multi-model switching control (e.g. see [26], [27], [28], [14], and $[13])$.

The study of the adaptive control of time-varying systems has not been as successful. Of course, the underlying motivation for adaptive control is to handle possibly rapidly varying parameters. The early adaptive control solutions were unable to handle fast timevariations. Much effort was devoted to resolving this problem; however, the results were 
mixed. With some modifications, many of the classical adaptive controllers have been proven to tolerate slow (or infrequent) time variations in the plant parameters (e.g. see [17], [19], [36], [39], [8], and [9]). The results for the systems with rapid time-variations were limited: either the form of the time-variations (or at least of the fast terms) were assumed to be known (e.g. see [37] and [35]) or only the plants with stable zero dynamics (the time-varying counterpart of minimum phase) were considered (e.g. see [1], [20], [15], [12], [18], and [23]). There are no results which deal with rapidly time-varying parameters in systems with unstable zero dynamics, and only a few which tolerate moderate parameter variations (e.g. see [40], [38], [24]).

Gain scheduling is an area that developed in parallel to adaptive control. The core idea in gain scheduling is to utilise linear controller design techniques to address nonlinear problems. The topic has started re-gaining interest in the 1990's (e.g. see [31] and [34]) after a few decades of relative silence. Many different design notions exist for gain scheduling such as pre-compensating a nonlinear gain with an inverse gain function or switching gain values according to operating conditions or even at pre-set times. The most common interpretation is that of continuously varying the controller coefficients based on the current value of a scheduling variable, which may be either an exogenous or endogenous signal with respect to the plant. The key aspect is that the scheduling variable is measurable and is available to the controller for feedback. The typical approach is to convert a nonlinear plant into a linear parameter varying (LPV) system by either Jacobian linearization about a family of equilibrium points or by absorbing a nonlinearity into the time-varying parameter. This design process usually results in a family of LTI controllers. Traditionally the design is such that the desired performance is achieved for each fixed value of the parameter and typically the stability is preserved only locally and in slow-variation setting. The control of LPV systems has attracted much interest and several approaches have been developed recently. Of special significance is the invariant set approach presented in [2], [3], [4], [6], [5], where it is shown that polyhedral Lyapunov functions and associated geometrically intuitive methods can be used for controller synthesis.

In [5] it is shown that if certain conditions are satisfied, then an observer based controller can be constructed such that the closed loop system is stable under arbitrarily fast timevariations in the parameter. This is true under a number of assumptions including the following: (i) the set of possible parameter values is compact and (ii) the parameters are measurable. While the presented results are very powerful, the major shortcoming of this work is that the conditions under which a controller can be constructed are very hard to check in general and are "subject to complexity concerns" as pointed out in [33]. As a result, it is practically impossible to identify a class of systems for which this method could apply and for which a simpler solution does not already exist. Assumption (ii) is also an 
issue when considered from the point of view of adaptive control.

\subsection{Purpose}

The goal of this thesis is to come up with the adaptive controller design that could stabilize a plant with possibly unstable zero dynamics under fast time-variations. This will be achieved primarily through extending the work on gain scheduling in [5]. We will only consider a scalar parameter uncertainty, but assumption (ii) will be relaxed. Recall that in the Certainty Equivalence approach, the key idea is to periodically estimate the parameters and update the control signal under the assumption that these estimates are exact. Here we adopt this approach and design a periodic controller. Each period is divided into the estimation and control phases. Once the necessary state and parameter estimates are calculated in the estimation phase, an appropriately scaled control signal is applied in the second phase. The estimation procedure similar to the one suggested in [21] will be used and the obtained estimates will be plugged into the perfect information control law from [5]. We will require an upper bound on the magnitude of the derivative of the parameter to make the estimation possible. This bound can be quite large and, therefore, such assumption is not very restrictive. Furthermore, since we are considering a one dimensional case, the set of possible parameter values is the union of a finite number of disjoint closed intervals. Hence, in order for the parameter to move from one interval to another, we will need to allow for occasional jumps in the parameter values, which will slightly complicate the proofs.

\subsection{Organization}

The mathematical preliminaries are presented in the next chapter. In Chapter 3 we define the problem and state the necessary assumptions. The noise free case is presented in Chapter 4 to gain intuition into the problem. We consider the system in the presence of noise in Chapter 5, where many results are adopted from the earlier chapter. Chapter 6 presents an example and a simulation. Finally, Chapter 7 concludes the thesis by summarizing the main achievements and outlining future work. 


\section{Chapter 2}

\section{Mathematical Preliminaries}

Let $\mathbb{Z}$ denote the set of integers, $\mathbb{Z}^{+}$represent the set of non-negative integers, $\mathbb{N}$ denote the set of natural numbers, $\mathbb{R}$ denote the set of real numbers, and $\mathbb{R}^{+}$represent the set of non-negative real numbers.

We will use the 1-norm to measure the size of a vector. For a given $x \in \mathbb{R}^{n}$,

$$
\|x\|:=\sum_{i=1}^{n}\left|x_{i}\right| .
$$

The corresponding induced norm of a matrix $A \in \mathbb{R}^{m \times n}$ is defined in a usual manner:

$$
\|A\|=\sup _{\|x\| \neq 0} \frac{\|A x\|}{\|x\|}
$$

The 1-norm of a matrix also has a special significance - it represents the maximum column sum of the elements of the matrix:

$$
\|A\|=\max _{j} \sum_{i=1}^{m}\left|a_{i, j}\right| .
$$

For a given set $\mathcal{S} \subseteq \mathbb{R}^{m \times n}$, we let $P C(\mathcal{S})$ denote the set of all piecewise continuous functions $f: \mathbb{R}^{+} \rightarrow \mathcal{S}$. To measure the size of such functions, we define

$$
\|f\|_{\infty}:=\sup _{t \in \mathbb{R}^{+}}\|f(t)\|
$$


We let $A C(\mathcal{S})$ denote the set of all absolutely continuous functions of the form $f: \mathbb{R}^{+} \rightarrow \mathcal{S}$. We say $f \in P C(\mathcal{S})$ is piecewise smooth on $[a, b] \subset \mathbb{R}^{+}$if there exists a finite set of points $\left\{x_{i}\right\}$

$$
a=x_{1}<x_{2}<\cdots<x_{k}=b
$$

such that, on each interval $\left(x_{i}, x_{i+1}\right), i=1,2, \ldots, k-1$, we have that $f$ and $\dot{f}$ are continuous and bounded, and they both have finite limits as $x \rightarrow x_{i}$ and $x \rightarrow x_{i+1}$. We say that $f \in P C(\mathcal{S})$ is piecewise smooth (and we write $f \in P S(\mathcal{S})$ ) if it is piecewise smooth on every finite interval $[a, b] \subset \mathbb{R}^{+}$. With $T_{0}>0$, we let $P S\left(\mathcal{S}, T_{0}\right)$ denote the set of $f \in P S(\mathcal{S})$ for which every discontinuity of $\left[\begin{array}{l}f \\ \dot{f}\end{array}\right]$ are at least $T_{0}$ time units apart.

For a given set $\mathcal{S} \subseteq \mathbb{R}$ of the form $\mathcal{S}:=\left[\underline{s}_{1}, \bar{s}_{1}\right] \cup\left[\underline{s}_{2}, \bar{s}_{2}\right] \cup \cdots \cup\left[\underline{s}_{q}, \bar{s}_{q}\right]$, we define a projection function $\Pi_{\mathcal{S}}: \mathbb{R} \rightarrow \mathcal{S}$ as follows: for $a \in \mathbb{R}$ we have

$$
\Pi_{\mathcal{S}}(a):=\left\{\begin{array}{cl}
a & \text { if } a \in \mathcal{S} \\
\underline{s}_{1} & \text { if } a<\underline{s}_{1} ; \\
\bar{s}_{j} & \text { if } a \in\left(\bar{s}_{j}, \frac{1}{2}\left(\bar{s}_{j}+\underline{s}_{j+1}\right)\right] \text { for } j=1,2, \ldots, q-1 ; \\
\underline{s}_{j+1} & \text { if } a \in\left(\frac{1}{2}\left(\bar{s}_{j}+\underline{s}_{j+1}\right), \underline{s}_{j+1}\right) \text { for } j=1,2, \ldots, q-1 ; \\
\bar{s}_{q} & \text { if } a>\bar{s}_{q} .
\end{array}\right.
$$

In several places throughout the thesis we use the Taylor series for the exponential: for $T>0$ and $a>0$,

$$
e^{-a T}=1-a T+\frac{a^{2} T^{2}}{2 !}-\frac{a^{3} T^{3}}{3 !}+\ldots,
$$

the obvious consequence of which is that $1-a T<e^{-a T}$. 


\section{Chapter 3}

\section{Problem Formulation}

\subsection{Adaptive Control of Time-Varying Systems}

In adaptive control of time-varying systems, a typical single-input single-output (SISO) plant can be described by the following state-space equations:

$$
\begin{aligned}
\dot{x}(t) & =A(t) x(t)+B(t) u(t), \quad x\left(t_{0}\right)=x_{0} \\
y(t) & =C(t) x(t),
\end{aligned}
$$

where $x(t) \in \mathbb{R}^{n}$ is the plant state, $u(t) \in \mathbb{R}$ is the input to the plant and $y(t) \in \mathbb{R}$ is the plant output. If the form of the time-variation is unknown, as is in our case, it is often necessary to include a bound on the rate of the variations, i.e. $\|\dot{A}(t)\|,\|\dot{B}(t)\|,\|\dot{C}(t)\|$ are uniformly bounded. The set of admissible triples $(A(t), B(t), C(t))$ is labeled $\mathcal{P}$.

We will consider standard output-feedback controllers of the form

$$
u=K y .
$$

In a realistic scenario there is noise injected into the system from the outside environment when the controller is connected to the plant, modeled by introducing the noise signals at the input and output interfaces. If we define $d$ and $w$ to be the noise signals, the input into the plant becomes $u_{d}=u+d$. Accordingly, the measured output available to the controller is $y_{w}=y+w$. Refer to Figure 3.1 for the block diagram of the closed loop system.

When the plant's initial condition is zero, i.e. $x_{0}=0$, for every $P \in \mathcal{P}$ we let $\Psi(P)$ to be the closed loop map from $\left[\begin{array}{l}d \\ w\end{array}\right]$ to $\left[\begin{array}{l}y \\ u\end{array}\right]$. 


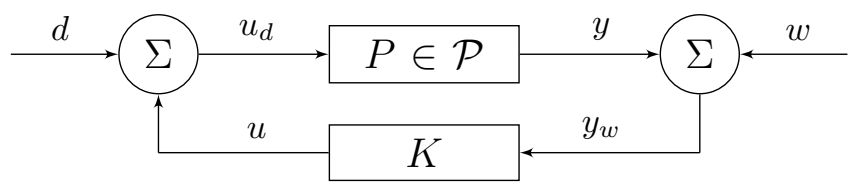

Figure 3.1: Closed loop system considered in this thesis.

Definition 1 (Stability). We say that $K$ stabilizes $\mathcal{P}$ if $\Psi(P)$ is uniformly bounded, i.e.

$$
\sup _{P \in \mathcal{P}}\|\Psi(P)\|<\infty .
$$

The goal of this thesis is to design $K$ that stabilizes $\mathcal{P}$. We will be considering fast time-variations of the plant and in our approach we will adopt a result from the area of gain-scheduling, which is presented in the next section.

\subsection{The Gain Scheduling Result}

Consider a liner parameter varying (LPV) system of the form

$$
\begin{aligned}
\dot{x}(t) & =A(\alpha(t)) x(t)+B(\alpha(t)) u(t) \\
y(t) & =C(\alpha(t)) x(t)
\end{aligned}
$$

where $x(t) \in \mathbb{R}^{n}$ is the plant state and $u(t)$ and $y(t)$ are scalar input and output signals respectively. The parameter $\alpha(t)$ is a time-varying uncertain index parameter that takes values in a compact set $\mathcal{A} \subset \mathbb{R}^{p}$; its values are measurable and available to the controller for feedback. The set of all admissible functions for $\alpha(t)$ is $P C(\mathcal{A}) . A(\alpha), B(\alpha)$ and $C(\alpha)$ are assumed to be continuous functions of $\alpha$.

We will require a few definitions to formalize the analysis that will follow.

Definition 2 (LPV Exponential Stability). Given $\mathcal{A}$, the system

$$
\dot{x}(t)=A(\alpha(t)) x(t), \quad x\left(t_{0}\right)=x_{0}
$$

is said to be $L P V$ exponentially stable if there exists a $\gamma \geq 1$ and $\lambda>0$ such that for every $t_{0} \in \mathbb{R}, x_{0} \in \mathbb{R}^{n}$, and $\alpha \in P C(\mathcal{A})$, the solution of (3.6) satisfies

$$
\|x(t)\| \leq \gamma e^{-\lambda\left(t-t_{0}\right)}\left\|x\left(t_{0}\right)\right\|, \quad \text { for } t \geq t_{0} .
$$


Definition 3 (Class $\left.\mathcal{H}_{1}\right)$. We define the square matrix $H(\alpha)$, a continuous function of $\alpha$, to be of class $\mathcal{H}_{1}$ if there exists a $\bar{\tau}>0$ such that $\|I+\tau H(\alpha)\|<1$ for all $\tau \in(0, \bar{\tau})$ and $\alpha \in \mathcal{A}$.

Proposition 1. Consider a matrix $H(\alpha) \in \mathcal{H}_{1}$. There exist $\bar{\lambda}>0$ and $\bar{T}>0$ such that for all $\lambda \in(0, \bar{\lambda})$ and $T \in(0, \bar{T})$ the following holds:

$$
\|I+T H(\alpha)\|<1-\lambda T \text { for all } \alpha \in \mathcal{A} .
$$

Proof. Refer to Appendix A.

Definition 4 (Class $\left.\mathcal{H}_{\infty}\right)$. We define the square matrix $H(\alpha)$, a continuous function of $\alpha$, to be of class $\mathcal{H}_{\infty}$ if there exists a $\bar{\tau}>0$ such that $\|I+\tau H(\alpha)\|_{\infty}<1^{1}$ for all $\tau \in(0, \bar{\tau})$ and $\alpha \in \mathcal{A}$.

Proposition 2. Consider a matrix $H(\alpha) \in \mathcal{H}_{\infty}$. There exist $\bar{\lambda}>0$ and $\bar{T}>0$ such that for all $\lambda \in(0, \bar{\lambda})$ and $T \in(0, \bar{T})$ the following holds:

$$
\|I+T H(\alpha)\|_{\infty}<1-\lambda T \text { for all } \alpha \in \mathcal{A} .
$$

Proof. Refer to Appendix A.

Proposition 3. Given $\mathcal{A}$, if $H(\alpha) \in \mathcal{H}_{1}$ or $H(\alpha) \in \mathcal{H}_{\infty}$, then the system

$$
\dot{x}(t)=H(\alpha(t)) x(t), \quad x\left(t_{0}\right)=x_{0}
$$

is LPV exponentially stable.

Proof. Refer to Appendix A.

Definition 5 (Output Feedback Controller). Given $\mathcal{A}$ and continuous functions $F(\cdot)$, $G(\cdot), H(\cdot)$, and $K(\cdot)$, the LPV controller

$$
\begin{aligned}
\dot{z}(t) & =F(\alpha(t)) z(t)+G(\alpha(t)) y(t) \\
u(t) & =H(\alpha(t)) z(t)+K(\alpha(t)) y(t)
\end{aligned}
$$

$L P V$ exponentially stabilizes (3.4) and (3.5) if the closed loop system

$$
\left[\begin{array}{c}
\dot{x}(t) \\
\dot{z}(t)
\end{array}\right]=\left[\begin{array}{cc}
A(\alpha(t))+B(\alpha(t)) K(\alpha(t)) C(\alpha(t)) & B(\alpha(t)) H(\alpha(t)) \\
G(\alpha(t)) C(\alpha(t)) & F(\alpha(t))
\end{array}\right]\left[\begin{array}{l}
x(t) \\
z(t)
\end{array}\right]
$$

is LPV exponentially stable.

\footnotetext{
${ }^{1}$ Here $\|\cdot\|_{\infty}$ has a meaning of an infinity norm on $\mathbb{R}^{m \times n}$ defined in the usual way.
} 
We are now ready to present a slightly modified ${ }^{2}$ version of Theorem 3.1 from [5], which will be used to construct our adaptive controller.

Theorem 1. The LPV system (3.4)-(3.5) is LPV exponentially stabilizable via an output feedback controller of the form (3.11)-(3.12) if there exists a matrix $P(\alpha) \in \mathcal{H}_{1}$, a full row-rank $n \times m$ matrix $X$, a $1 \times m$ matrix $U(\alpha)$ as well as $Q(\alpha) \in \mathcal{H}_{\infty}$, a full column-rank $l \times n$ matrix $R$ and a $l \times 1$ matrix $L(\alpha)$ such that equations

$$
\begin{aligned}
A(\alpha) X+B(\alpha) U(\alpha) & =X P(\alpha) \\
R A(\alpha)+L(\alpha) C(\alpha) & =Q(\alpha) R
\end{aligned}
$$

are satisfied for all $\alpha \in \mathcal{A}$.

Proof. Assume that there exist matrices $X, R, P(\alpha), Q(\alpha), U(\alpha)$ and $L(\alpha)$ in the appropriate sets for which conditions (3.14) and (3.15) are satisfied. Since R has full column rank, we let matrix $M$ be any left inverse of $R$, i.e. $M R:=I$. We first introduce the matrix $Z$ to be any matrix satisfying the property that $\left[\begin{array}{l}X \\ Z\end{array}\right]$ is invertible. Now we can define matrix $V(\alpha)$ as follows:

$$
V(\alpha):=Z P(\alpha) .
$$

Consider a state estimator of the form

$$
\begin{aligned}
& \dot{r}(t)=Q(\alpha(t)) r(t)-L(\alpha(t)) y(t)+R B(\alpha(t)) u(t) \\
& \hat{x}(t)=M r(t)
\end{aligned}
$$

and a state-feedback controller:

$$
\begin{aligned}
& \dot{z}(t)=F(\alpha(t)) z(t)+G(\alpha(t)) \hat{x}(t) \\
& u(t)=H(\alpha(t)) z(t)+K(\alpha(t)) \hat{x}(t) .
\end{aligned}
$$

The controller gains $F(\alpha), G(\alpha), H(\alpha)$, and $K(\alpha)$ are calculated as follows:

$$
\left[\begin{array}{ll}
K(\alpha) & H(\alpha) \\
G(\alpha) & F(\alpha)
\end{array}\right]=\left[\begin{array}{l}
U(\alpha) \\
V(\alpha)
\end{array}\right]\left[\begin{array}{l}
X \\
Z
\end{array}\right]^{-1}
$$

or

$$
\left[\begin{array}{ll}
K(\alpha) & H(\alpha) \\
G(\alpha) & F(\alpha)
\end{array}\right]\left[\begin{array}{l}
X \\
Z
\end{array}\right]=\left[\begin{array}{l}
U(\alpha) \\
V(\alpha)
\end{array}\right]
$$

\footnotetext{
${ }^{2}$ Here we consider the sufficient conditions for stabilizability whereas the original theorem claims the same conditions are also necessary.
} 
If we analyse the differential equation corresponding to $\left[\begin{array}{c}x(t) \\ z(t) \\ r(t)\end{array}\right]$, it will be hard to gain insight. Hence, we will introduce a new variable $s(t)$ :

$$
s(t):=R x(t)-r(t) .
$$

Note that

$$
\begin{aligned}
M s(t) & =M R x(t)-M r(t) \\
& =x(t)-\hat{x}(t) .
\end{aligned}
$$

Therefore, as $s(t)$ approaches zero, the state estimation error becomes smaller as well:

$$
s(t) \rightarrow 0 \Longrightarrow \hat{x}(t) \rightarrow x(t)
$$

On that basis, we will analyse the differential equation corresponding to $\left[\begin{array}{l}x(t) \\ z(t) \\ s(t)\end{array}\right]$. Now we derive the dynamics of each state variable in the closed loop; to reduce notational clutter, we drop the dependence of $\alpha$ on $t$ :

$$
\begin{aligned}
\dot{x}(t) & =A(\alpha) x(t)+B(\alpha) u(t) \\
& =A(\alpha) x(t)+B(\alpha)[H(\alpha) z(t)+K(\alpha) \hat{x}(t)] \\
& =A(\alpha) x(t)+B(\alpha) H(\alpha) z(t)+B(\alpha) K(\alpha)[x(t)-M s(t)] \\
& =[A(\alpha)+B(\alpha) K(\alpha)] x(t)+B(\alpha) H(\alpha) z(t)-B(\alpha) K(\alpha) M s(t) ; \\
\dot{z}(t) & =F(\alpha) z(t)+G(\alpha) \hat{x}(t) \\
& =F(\alpha) z(t)+G(\alpha)[x(t)-M s(t)] \\
& =G(\alpha) x(t)+F(\alpha) z(t)-G(\alpha) M s(t) ; \\
\dot{s}(t) & =R \dot{x}(t)-\dot{r}(t) \\
& =R[A(\alpha) x(t)+B(\alpha) u(t)]-[Q(\alpha) r(t)-L(\alpha) y(t)+R B(\alpha) u(t)] \\
& =R A(\alpha) x(t)+R B(\alpha) u(t)-Q(\alpha)[R x(t)-s(t)]+L(\alpha) C(\alpha) x(t)-R B(\alpha) u(t) \\
& =\underbrace{[R A(\alpha)+L(\alpha) C(\alpha)-Q(\alpha) R] x(t)+Q(\alpha) s(t)}_{=0 \text { from }(3.15)} \\
& =Q(\alpha) s(t) .
\end{aligned}
$$


Combining the above equations, the state space representation of the closed loop system will take the following form:

$$
\left[\begin{array}{c}
\dot{x}(t) \\
\dot{z}(t) \\
\dot{s}(t)
\end{array}\right]=\left[\begin{array}{cc|c}
A(\alpha)+B(\alpha) K(\alpha) & B(\alpha) H(\alpha) & -B(\alpha) K(\alpha) M \\
G(\alpha) & F(\alpha) & -G M \\
\hline 0 & 0 & Q(\alpha)
\end{array}\right]\left[\begin{array}{c}
x(t) \\
z(t) \\
s(t)
\end{array}\right] .
$$

For LPV exponential stability we require that the blocks on the main diagonal correspond to LPV exponentially stable systems. Since $Q(\alpha)$ lies in $\mathcal{H}_{\infty}$, by Proposition 3 we know that the corresponding system is LPV exponentially stable. We need to show that the system corresponding to the upper left block is also LPV exponentially stable.

By combining (3.22) with (3.14) we obtain

$$
\begin{aligned}
A(\alpha) X+B(\alpha)[K(\alpha) X+H(\alpha) Z] & =X P(\alpha) \\
G(\alpha) X+F(\alpha) Z & =V(\alpha) .
\end{aligned}
$$

Also, from (3.16) we know that $V(\alpha)=Z P(\alpha)$, so the above two equations transform into

$$
\left[\begin{array}{cc}
A(\alpha)+B(\alpha) K(\alpha) & B(\alpha) H(\alpha) \\
G(\alpha) & F(\alpha)
\end{array}\right]\left[\begin{array}{c}
X \\
Z
\end{array}\right]=\left[\begin{array}{c}
X \\
Z
\end{array}\right] P(\alpha)
$$

The matrix $\left[\begin{array}{l}X \\ Z\end{array}\right]$ is invertible by construction, which means that the upper left block in (3.24) is equal to a similarity transformation of $P(\alpha)$ :

$$
\left[\begin{array}{cc}
A(\alpha)+B(\alpha) K(\alpha) & B(\alpha) H(\alpha) \\
G(\alpha) & F(\alpha)
\end{array}\right]=\left[\begin{array}{c}
X \\
Z
\end{array}\right] P(\alpha)\left[\begin{array}{c}
X \\
Z
\end{array}\right]^{-1}
$$

Since the system corresponding to $P(\alpha)$ is LPV exponentially stable by Proposition 3 and stability is preserved under a similarity transformation, the closed loop system in (3.24) is LPV exponentially stable.

A few important observations should be made about equations (3.14) and (3.15). Given $A(\alpha), B(\alpha)$, and $C(\alpha)$, it is very difficult to find matrices $X, U(\alpha), P(\alpha), R, L(\alpha)$, and $Q(\alpha)$ that satisfy (3.14)-(3.15); in fact, there is no known way of knowing if there exists a solution without actually finding one. The fact that the dimensions of $X$ and $R$ are not fixed $-m$ and $l$ can be any numbers larger than or equal to $n$, makes the problem that much more difficult. This results in computational complexity concerns mentioned 
in [33]. Further research is required to formally characterize the difficulty of solving these equations. Note however, that the two equations are subject to the separation principle. It is clear from equations (3.17)-(3.20) that (3.14) is a condition for stabilizability, whereas (3.15) is a detectability condition. This means that we can construct our own state and parameter estimators and once we solve (3.14), a stabilizing controller can be constructed by suitably modifying equations (3.19) and (3.20).

Remark 1. Since matrices $A(\alpha), B(\alpha), C(\alpha), P(\alpha)$, and $Q(\alpha)$ are assumed to be continuous, if $B(\alpha)$ has full column rank, then the matrix $U(\alpha)$ of Theorem 1 must be continuous. Similarly, if $C(\alpha)$ has full row rank, then the matrix $L(\alpha)$ of Theorem 1 must be continuous.

Remark 2. If we can find a solution to equation (3.14) where $X$ is square, it must be that $Z$ is a null matrix. This immediately leads to a much simplified control law:

$$
u(t)=U(\alpha(t)) X^{-1} \hat{x}(t)
$$

Notice, that the knowledge of $P(\alpha)$ is no longer required. This greatly reduces the computational complexity of finding and implementing a controller.

\subsection{The Proposed Approach}

In this thesis we will consider systems of the form

$$
\begin{aligned}
\dot{x}(t) & =A(\alpha(t)) x(t)+B(\alpha(t)) u(t), \quad x(0)=x_{0} \\
y(t) & =C x(t),
\end{aligned}
$$

where $x(t) \in \mathbb{R}^{n}$ is the plant state, $u(t) \in \mathbb{R}$ is the input to the plant, and $y(t) \in \mathbb{R}$ is the plant output. The parameter $\alpha(t)$ takes values in a compact set $\mathcal{A} \subset \mathbb{R}$, which is assumed to be composed of $q \in \mathbb{N}$ disjoint closed intervals. Since $\mathcal{A}$ is compact, there exist $\underline{\alpha}=\min \mathcal{A}$ and $\bar{\alpha}=\max \mathcal{A}$. Since the case of $n=1$ corresponds to a minimum phase

plant which is well understood (see [1], [20], [15], [12], [18], and [23]), here we will assume that $n \geq 2$.

In our analysis we consider a situation where the values of the state and the time-varying parameter are not available to the controller for feedback. Hence, we will use a nonlinear sampled-data periodic controller that will estimate the state and the parameter in each period and use these estimates to generate a stabilizing control signal. The main idea for the estimation procedure is to estimate the system's Markov parameters in a manner 
similar to that described in [21]. We will rely on the following set of assumptions to allow the state and parameter estimation and to ascertain that the system is stabilizable. The first two are very natural:

- Assumption 1: $(A, B)(\alpha)$ is controllable for all $\alpha \in \mathcal{A}$.

- Assumption 2: $A(\alpha)$ and $B(\alpha)$ are absolutely continuous functions of $\alpha$ and there exist $\delta_{A}$ and $\delta_{B}$ so that

$$
\delta_{A}:=\operatorname{ess} \sup _{\alpha \in \mathcal{A}}\left\|\frac{d A(\alpha)}{d \alpha}\right\|<\infty \quad \text { and } \quad \delta_{B}:=\operatorname{ess} \sup _{\alpha \in \mathcal{A}}\left\|\frac{d B(\alpha)}{d \alpha}\right\|<\infty .
$$

Since $\mathcal{A}$ is compact and $A(\alpha)$ and $B(\alpha)$ are absolutely continuous, there exist positive constants

$$
c_{a}:=\sup _{\alpha \in \mathcal{A}}\|A(\alpha)\|<\infty \quad \text { and } \quad c_{b}:=\sup _{\alpha \in \mathcal{A}}\|B(\alpha)\|<\infty .
$$

The next assumption allows us to estimate the plant state even when the parameter $\alpha(t)$ is time-varying:

- Assumption 3: The observability matrix $\mathcal{O}(C, A(\alpha)):=\left[\begin{array}{c}C \\ C A(\alpha) \\ \vdots \\ C A^{n-1}(\alpha)\end{array}\right]$ is independent of the parameter $\alpha$ and invertible.

We will use ideas from [21] to estimate plant Markov parameters using a probing signal. To this end, we impose: 


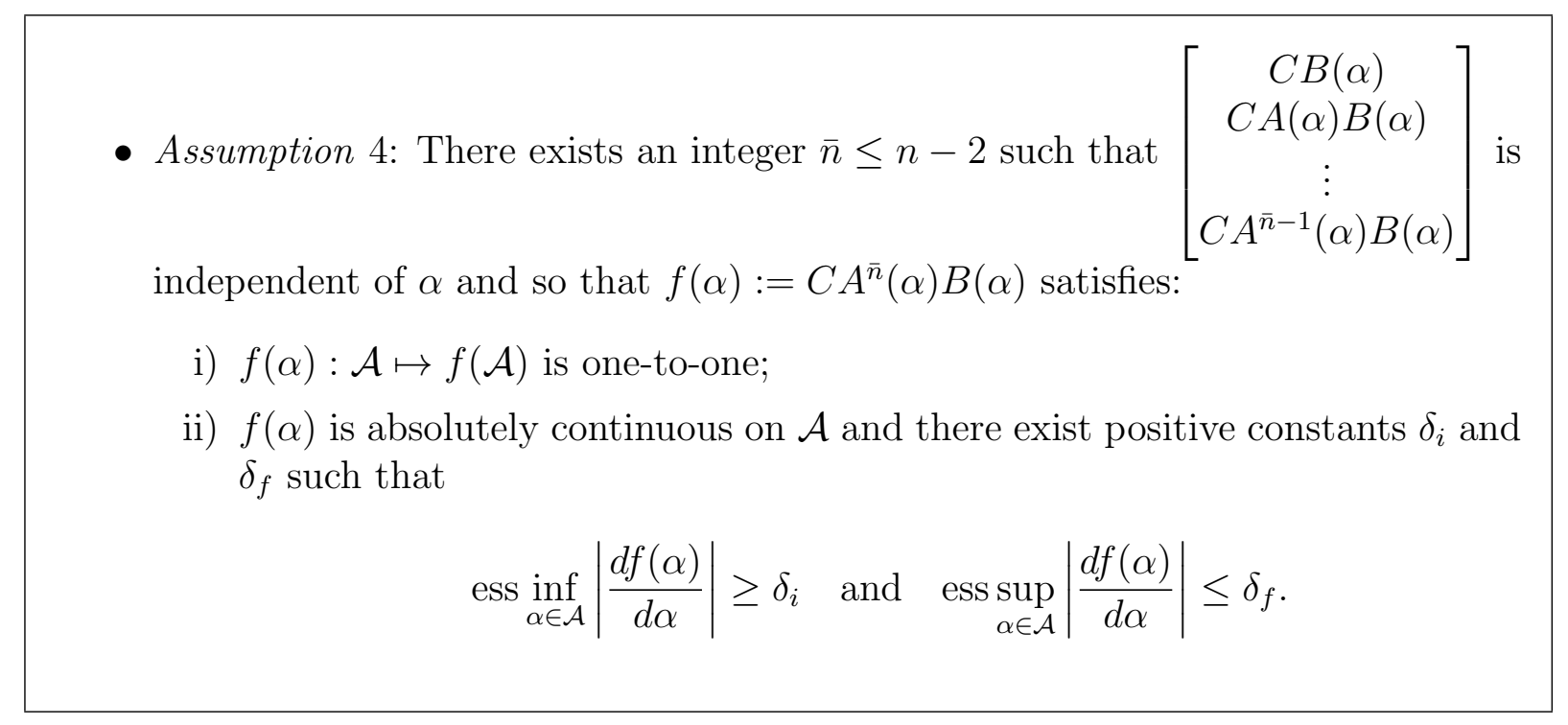

Assumption 4 i) implies, in particular, that $f(\mathcal{A})$ has $q$ disjoint intervals, which we label

$$
f(\mathcal{A})=\left[\underline{f}_{1}, \bar{f}_{1}\right] \cup\left[\underline{f}_{2}, \bar{f}_{2}\right] \cup \cdots \cup\left[\underline{f}_{q}, \bar{f}_{q}\right]=: \mathcal{F} .
$$

We plan to use a sampled-data controller, so we cannot allow $\alpha(t)$ to move arbitrarily fast, though we allow it to have an occasional jump:

- Assumption 5:

i) There exists a $T_{0}>0$ so that $\alpha \in P S\left(\mathcal{A}, T_{0}\right)$;

ii) There exists a $\delta_{\alpha}$ so that

$$
\operatorname{ess} \sup _{\alpha \in \mathcal{A}}|\dot{\alpha}(t)| \leq \delta_{\alpha}
$$

Given that our techniques will be based on an adaptive version of gain scheduling, we impose 
- Assumption 6: There exists an absolutely continuous matrix $P(\alpha) \in \mathcal{H}_{1}$, a full row-rank $n \times m$ matrix $X$, and a $1 \times m$ matrix $U(\alpha)$ such that equation (3.14) is satisfied and so that

$$
\operatorname{ess} \sup _{\alpha \in \mathcal{A}}\left\|\frac{d P(\alpha)}{d \alpha}\right\|<\infty .
$$

The ramification of Assumptions 1, 2 and 6 is that by Remark 1, $U(\alpha)$ is also absolutely continuous with

$$
\operatorname{ess} \sup _{\alpha \in \mathcal{A}}\left\|\frac{d U(\alpha)}{d \alpha}\right\|<\infty .
$$

This means that matrices $F(\alpha), G(\alpha), H(\alpha)$, and $K(\alpha)$ are absolutely continuous by construction - see equations (3.16) and (3.21) and also have bounded derivatives. Hence, we can introduce constants

$$
\begin{gathered}
\delta_{F}:=\operatorname{ess} \sup _{\alpha \in \mathcal{A}}\left\|\frac{d F(\alpha)}{d \alpha}\right\|<\infty, \quad \delta_{G}:=\operatorname{ess} \sup _{\alpha \in \mathcal{A}}\left\|\frac{d G(\alpha)}{d \alpha}\right\|<\infty, \\
\delta_{H}:=\operatorname{ess} \sup _{\alpha \in \mathcal{A}}\left\|\frac{d H(\alpha)}{d \alpha}\right\|<\infty, \quad \delta_{K}:=\operatorname{ess} \sup _{\alpha \in \mathcal{A}}\left\|\frac{d K(\alpha)}{d \alpha}\right\|<\infty .
\end{gathered}
$$

Since $\mathcal{A}$ is compact and $F(\alpha), G(\alpha), H(\alpha)$, and $K(\alpha)$ are absolutely continuous, there exist positive constants

$$
\begin{aligned}
& c_{f}:=\sup _{\alpha \in \mathcal{A}}\|F(\alpha)\|<\infty, c_{g}:=\sup _{\alpha \in \mathcal{A}}\|G(\alpha)\|<\infty, \\
& c_{h}:=\sup _{\alpha \in \mathcal{A}}\|H(\alpha)\|<\infty, \quad c_{k}:=\sup _{\alpha \in \mathcal{A}}\|K(\alpha)\|<\infty .
\end{aligned}
$$

As we mentioned in the previous section, obtaining examples which satisfy Assumption 6 is difficult. Fortunately, good examples do exist and are discussed in Chapter 6. 


\section{Chapter 4}

\section{Noise Free Case}

In this chapter we will consider system (3.26)-(3.27) without the noise inputs, i.e. we set $d(t)=0$ and $w(t)=0$ in Figure 3.1. This is done in order to gain intuition into the analysis of the system before delving into the more complex noisy case. We will propose the techniques to estimate the state and the parameter in every period. Upper bounds on the estimation errors will be derived and used to show that the closed loop system depicted in Figure 4.1 is stable. Note that the sampler $(\mathrm{S})$ and a zero-order hold $(\mathrm{H})$ blocks operate with a period of $h$ seconds. Hence, we define the sampled output as

$$
y[i]:=y(i h), \quad i \in \mathbb{Z}^{+} .
$$

We will use a sampled-data periodic controller with a period $T:=N h$ to stabilize the plant. A distinction should be made between the base sampling period $(h)$ and the period of the controller $(T)$, which represents the time between the successive control law updates. We will associate the index $i \in \mathbb{Z}^{+}$with the sampling period and the index $k \in \mathbb{Z}^{+}$- with the controller period. Each period $T$ is divided into three phases with durations $T_{1}, T_{2}$, and $T_{3}$ respectively, such that $T=T_{1}+T_{2}+T_{3}$. During the first phase the plant state is estimated by allowing the system to run in open loop, i.e. $u(t)=0$ for $t \in\left[k T, k T+T_{1}\right.$ ), which yields $\hat{x}[k]$. We will calculate the parameter estimate, $\hat{\alpha}[k]$, in the second phase by exciting the system with a probing control input, i.e. $u(t)=u_{p}[k]$ when $t \in\left[k T+T_{1}, k T+T_{1}+T_{2}\right)$. The durations of the two phases are $T_{1}:=n h$ and $T_{2}:=(\bar{n}+2) h$. The reasoning behind these choices will become clear in the next two sections. Finally, a stabilizing control signal is applied in the third phase: $u(t)=u_{s}[k]$ for $t \in\left[k T+T_{1}+T_{2}, k T+T\right)$. To calculate $u_{s}[k]$ we will use suitably scaled discretized 


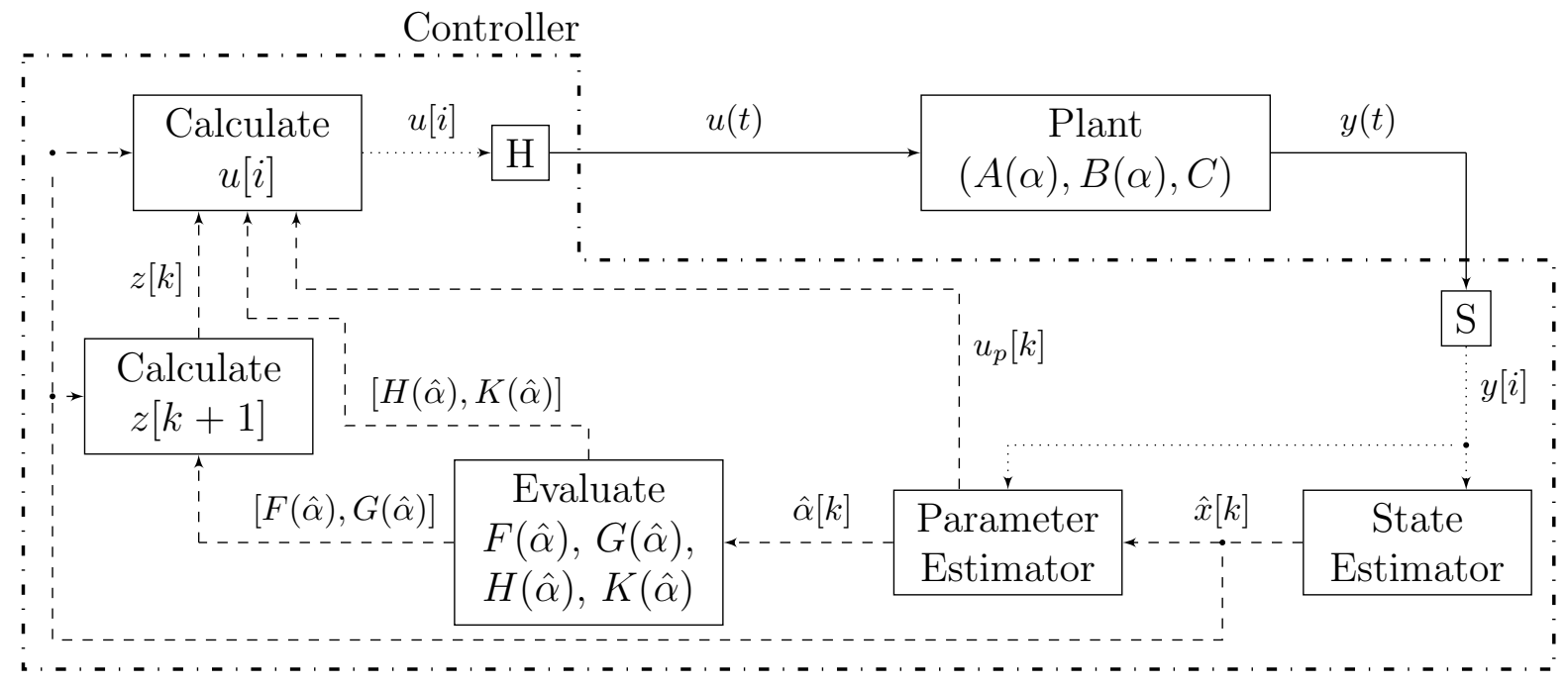

Figure 4.1: System setup when noise is not present.

versions of (3.19)-(3.20):

$$
\begin{aligned}
z[k+1] & =[I+T F(\hat{\alpha}[k])] z[k]+T G(\hat{\alpha}[k]) \hat{x}[k], \\
u_{s}[k] & =\frac{T}{T_{3}}[H(\hat{\alpha}[k]) z[k]+K(\hat{\alpha}[k]) \hat{x}[k]]-\frac{T_{2}}{T_{3}} u_{p}[k] .
\end{aligned}
$$

Defining the discrete time control signal as

$$
u[i]=\left\{\begin{array}{cl}
0 & i \in[k N, k N+n-1] \\
u_{p}[k] & i \in[k N+n, k N+n+\bar{n}+1] \\
u_{s}[k] & i \in[k N+n+\bar{n}+2, k N+N-1],
\end{array}\right.
$$

the controller output $u(t)$ is obtained by putting $u[i]$ through the zero-order hold as shown in Figure 4.1. The control signal during a typical period is depicted in Figure 4.2.

\subsection{State Estimation}

In order to obtain an estimate of the state in each period, $\hat{x}[k]$, we will allow the system to run in open loop for $T_{1}=n h$ seconds, i.e. we will set $u(t)=0$ for $t \in[k T, k T+n h)$. 


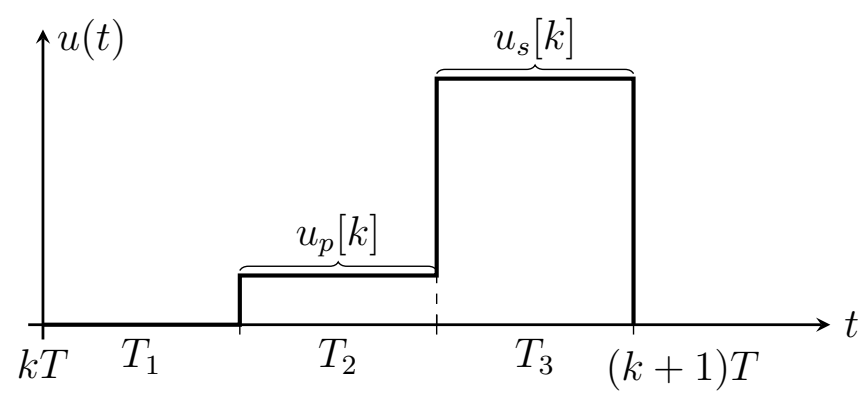

Figure 4.2: Control signal for $t \in[k T,(k+1) T)$.

This reduces system (3.26)-(3.27) to the following:

$$
\begin{aligned}
& \dot{x}(t)=A(\alpha(t)) x(t), \\
& y(t)=C x(t) .
\end{aligned}
$$

Differentiating $y(t)$ for $t \in(k T, k T+n h)$ yields:

$$
\dot{y}(t)=C \dot{x}(t)=C A(\alpha(t)) x(t) .
$$

Similarly,

$$
\ddot{y}(t)=\frac{d}{d t}[C A(\alpha(t))] x(t)+C A(\alpha(t)) \dot{x}(t)=C A^{2}(\alpha(t)) x(t),
$$

where we used Assumption 3 to observe that $\frac{d}{d t}[C A(\alpha(t))]=0$. Using the same logic, we can derive the expression for the $n$ 'th derivative of the output:

$$
y^{(n)}(t)=C A^{n}(\alpha(t)) x(t), \quad t \in(k T, k T+n h) .
$$

The derivatives of the output are useful in estimating the state since

$$
\left[\begin{array}{c}
y(t) \\
\dot{y}(t) \\
\vdots \\
y^{(n-1)}(t)
\end{array}\right]=\left[\begin{array}{c}
C \\
C A(\alpha(t)) \\
\vdots \\
C A^{n-1}(\alpha(t))
\end{array}\right] x(t)=\mathcal{O}(C, A(\alpha)) x(t), \quad t \in(k T, k T+n h) .
$$

Now we would like to express the first $n-1$ derivatives of $y(t)$ in terms of the output samples. To this end, we will integrate equation (4.5) to obtain the following:

$$
y^{(n-1)}(t)=y^{(n-1)}\left(k T^{+}\right)+\underbrace{\int_{k T}^{t} y^{(n)}(\tau) d \tau}_{=: \nu_{1}(t)} .
$$


We are interested in putting an upper bound on $\left|\nu_{1}(t)\right|$ :

$$
\begin{aligned}
\left|\nu_{1}(t)\right| & \leq \int_{k T}^{t}\left\|y^{(n)}(\tau)\right\| d \tau \\
& \leq \int_{k T}^{t}\|C\|\left\|A^{n}(\alpha(\tau))\right\|\|x(\tau)\| d \tau \\
& \leq\|C\| \int_{k T}^{t}\|A(\alpha(\tau))\|^{n}\|x(\tau)\| d \tau \\
& \leq\|C\| c_{a}^{n} \int_{k T}^{t}\|x(\tau)\| d \tau .
\end{aligned}
$$

The following lemma will be used to estimate an upper bound on $\|x(\tau)\|$.

Lemma 1. Given equation (3.26) and the accompanying assumptions and with $t_{0} \geq 0$, if we define

$$
\bar{u}:=\sup _{t_{0} \leq \tau \leq t}|u(\tau)|,
$$

there exists an upper bound on the size of the state:

$$
\|x(t)\| \leq\left[\left\|x\left(t_{0}\right)\right\|+\left(t-t_{0}\right) c_{b} \bar{u}\right] e^{c_{a}\left(t-t_{0}\right)}, \quad t \geq t_{0} .
$$

Proof. Refer to Appendix A.

We will proceed with deriving a bound on $\left|\nu_{1}(t)\right|$ by using Lemma 1 with $t_{0}=k T$ and $\bar{u}=0$ in $(4.8)$ :

Hence,

$$
\left\|\nu_{1}(t)\right\| \leq\|C\| c_{a}^{n} \int_{k T}^{t}\|x(k T)\| e^{c_{a}(\tau-k T)} d \tau .
$$

$$
\begin{aligned}
\left|\nu_{1}(t)\right| & \leq\|C\| c_{a}^{n} \int_{k T}^{t}\|x(k T)\| e^{c_{a} n h} d \tau \\
& =\|C\| c_{a}^{n} e^{c_{a} n h}\|x(k T)\|(t-k T), \quad t \in(k T, k T+n h) .
\end{aligned}
$$

We can write an expression for $y^{(n-2)}(t)$ by utilizing (4.7):

$$
\begin{aligned}
y^{(n-2)}(t) & =y^{(n-2)}\left(k T^{+}\right)+\int_{k T}^{t} y^{(n-1)}(\tau) d \tau \\
& =y^{(n-2)}\left(k T^{+}\right)+\int_{k T}^{t}\left[y^{(n-1)}\left(k T^{+}\right)+\nu_{1}(\tau)\right] d \tau \\
& =y^{(n-2)}\left(k T^{+}\right)+(t-k T) y^{(n-1)}\left(k T^{+}\right)+\underbrace{\int_{k T}^{t} \nu_{1}(\tau) d \tau}_{=: \nu_{2}(t)}, \quad t \in(k T, k T+n h),
\end{aligned}
$$


where the bound on $\nu_{2}(t)$ is easily derived:

$$
\begin{aligned}
\left|\nu_{2}(t)\right| & \leq \int_{k T}^{t}\left|\nu_{1}(\tau)\right| d \tau \\
& \leq \int_{k T}^{t}\|C\| c_{a}^{n} e^{c_{a} n h}\|x(k T)\|(\tau-k T) d \tau \\
& \leq\|C\| c_{a}^{n} e^{c_{a} n h}\|x(k T)\| \frac{(t-k T)^{2}}{2}, \quad t \in(k T, k T+n h) .
\end{aligned}
$$

We can continue in a similar fashion until we arrive at the expression for the output signal consisting of its derivatives:

$$
\begin{aligned}
y(t)= & y(k T)+(t-k T) \dot{y}\left(k T^{+}\right)+\frac{(t-k T)^{2}}{2 !} \ddot{y}\left(k T^{+}\right)+\cdots+\frac{(t-k T)^{n-1}}{(n-1) !} y^{(n-1)}\left(k T^{+}\right) \\
& +\nu_{n}(t), \quad t \in(k T, k T+n h),
\end{aligned}
$$

in which

$$
\left|\nu_{n}(t)\right| \leq\|C\| c_{a}^{n} e^{c_{a} n h}\|x(k T)\| \frac{(t-k T)^{n}}{n !}, \quad t \in(k T, k T+n h) .
$$

We can use (4.12) to generate expressions for each of the $n$ samples of the output: $y(k T), y(k T+$ $h), \ldots, y(k T+(n-1) h)$. Let us define the following vectors of samples:

$$
\mathcal{Y}_{k}:=\left[\begin{array}{c}
y(k T) \\
y(k T+h) \\
\vdots \\
y(k T+(n-1) h)
\end{array}\right] \text { and } \mathcal{V}_{k}:=\left[\begin{array}{c}
0 \\
\nu_{n}(k T+h) \\
\vdots \\
\nu_{n}(k T+(n-1) h)
\end{array}\right] \text {. }
$$

Then we can write

$$
\begin{aligned}
& \mathcal{Y}_{k}=\left[\begin{array}{c}
y(k T) \\
y(k T)+h \dot{y}\left(k T^{+}\right)+\frac{h^{2}}{2 !} \ddot{y}\left(k T^{+}\right)+\ldots+\frac{h^{n-1}}{(n-1) !} y^{(n-1)}\left(k T^{+}\right) \\
y(k T)+2 h \dot{y}\left(k T^{+}\right)+\frac{4 h^{2}}{2 !} \ddot{y}\left(k T^{+}\right)+\ldots+\frac{2^{n-1} h^{n-1}}{(n-1) !} y^{(n-1)}\left(k T^{+}\right) \\
\vdots \\
y(k T)+(n-1) h \dot{y}\left(k T^{+}\right)+\frac{(n-1)^{2} h^{2}}{2 !} \ddot{y}\left(k T^{+}\right)+\ldots+\frac{(n-1)^{n-1} h^{n-1}}{n-1 !} y^{(n-1)}\left(k T^{+}\right)
\end{array}\right]+\mathcal{V}_{k} \\
& =\left[\begin{array}{ccccc}
1 & 0 & 0 & \cdots & 0 \\
1 & h & \frac{h^{2}}{2 !} & \cdots & \frac{h^{n-1}}{(n-1) !} \\
1 & 2 h & \frac{4 h^{2}}{2 !} & \cdots & \frac{2^{n-1} h^{n}-1}{(n-1) !} \\
\vdots & \vdots & \vdots & \ddots & \vdots \\
1 & (n-1) h & \frac{(n-1)^{2} h^{2}}{2 !} & \cdots & \frac{(n-1)^{n-1} h^{n-1}}{(n-1) !}
\end{array}\right]\left[\begin{array}{c}
y(k T) \\
\dot{y}\left(k T^{+}\right) \\
\ddot{y}\left(k T^{+}\right) \\
\vdots \\
y^{(n-1)}\left(k T^{+}\right)
\end{array}\right]+\mathcal{V}_{k} \text {. }
\end{aligned}
$$


Let us define two new matrices:

$$
S_{n}:=\left[\begin{array}{ccccc}
1 & 0 & 0 & \ldots & 0 \\
1 & 1 & 1 & \cdots & 1 \\
1 & 2 & 4 & \ldots & 2^{n-1} \\
\vdots & \vdots & \vdots & \ddots & \vdots \\
1 & n-1 & (n-1)^{2} & \cdots & (n-1)^{n-1}
\end{array}\right]
$$

and

$$
Q_{n}(h):=\left[\begin{array}{ccccc}
1 & & & & \\
& h & & & \\
& & \frac{h^{2}}{2 !} & & \\
& & & \ddots & \\
& & & & \frac{h^{n-1}}{(n-1) !}
\end{array}\right] .
$$

It is now easy to see that

$$
\left[\begin{array}{ccccc}
1 & 0 & 0 & \ldots & 0 \\
1 & h & \frac{h^{2}}{2 !} & \cdots & \frac{h^{n-1}}{(n-1) !} \\
1 & 2 h & \frac{4 h^{2}}{2 !} & \ldots & \frac{2^{n-1} h^{n}-1}{(n-1) !} \\
\vdots & \vdots & \vdots & \ddots & \vdots \\
1 & (n-1) h & \frac{(n-1)^{2} h^{2}}{2 !} & \cdots & \frac{(n-1)^{n-1} h^{n-1}}{(n-1) !}
\end{array}\right]=S_{n} Q_{n}(h) .
$$

Consequently,

$$
\begin{aligned}
\mathcal{Y}_{k} & =S_{n} Q_{n}(h)\left[\begin{array}{c}
y(k T) \\
\dot{y}\left(k T^{+}\right) \\
\vdots \\
y^{(n-1)}\left(k T^{+}\right)
\end{array}\right]+\mathcal{V}_{k} \\
& =S_{n} Q_{n}(h) \mathcal{O}(C, A(\alpha)) x(k T)+\mathcal{V}_{k} .
\end{aligned}
$$

Note that $S_{n}$ is an $n$-square Vandermonde matrix with distinct parameters, and therefore is invertible (see Theorem 4.9 in [41]). We can determine the inverse of $Q_{n}(h)$ explicitly:

$$
Q_{n}^{-1}(h)=\left[\begin{array}{ccccc}
1 & & & & \\
& \frac{1}{h} & & & \\
& & \frac{2 !}{h^{2}} & & \\
& & & \ddots & \frac{(n-1) !}{h^{n-1}}
\end{array}\right] .
$$


It is easy to see that $Q_{n}^{-1}(h)$ is defined for all $h>0$. Finally, $\mathcal{O}(C, A(\alpha))$ is independent of $\alpha(t)$ and invertible by Assumption 3. On that basis, we will estimate the plant state as

$$
\hat{x}[k]:=\mathcal{O}^{-1}(C, A(\alpha)) Q_{n}^{-1}(h) S_{n}^{-1} \mathcal{Y}_{k} .
$$

Defining the discretized state as $x[k]:=x(k T)$, we can combine equations (4.17) and (4.19) to obtain a bound on the state estimation error:

$$
\begin{aligned}
\|\hat{x}[k]-x[k]\| & =\left\|\mathcal{O}^{-1}(C, A(\alpha)) Q_{n}^{-1}(h) S_{n}^{-1} \mathcal{V}_{k}\right\| \\
& \leq\left\|\mathcal{O}^{-1}(C, A(\alpha))\right\|\left\|Q_{n}^{-1}(h)\right\|\left\|S_{n}^{-1}\right\|\left\|\mathcal{V}_{k}\right\| .
\end{aligned}
$$

Clearly, $\left\|\mathcal{O}^{-1}(C, A(\alpha))\right\|$ and $\left\|S_{n}^{-1}\right\|$ are finite, but we will need to derive bounds for $\left\|Q_{n}^{-1}(h)\right\|$ and $\left\|\mathcal{V}_{k}\right\|$. From (4.18) it is clear that for $h \in(0,1), \frac{(n-1) !}{h^{n-1}}$ is the largest element of $Q_{n}^{-1}(h)$. Therefore, with our definition of the induced matrix norm, it is easy to see that

$$
\left\|Q_{n}^{-1}(h)\right\|=\frac{(n-1) !}{h^{n-1}} .
$$

We will use (4.13) to bound individual elements of $\mathcal{V}_{k}$ :

$$
\begin{aligned}
\left|\nu_{n}(k T+h)\right| & \leq \frac{h^{n}}{n !}\|C\| c_{a}^{n} e^{c_{a} n h}\|x[k]\|, \\
\left|\nu_{n}(k T+2 h)\right| & \leq \frac{2^{n} h^{n}}{n !}\|C\| c_{a}^{n} e^{c_{a} n h}\|x[k]\|, \\
& \vdots \\
\left|\nu_{n}(k T+(n-1) h)\right| & \leq \frac{(n-1)^{n} h^{n}}{n !}\|C\| c_{a}^{n} e^{c_{a} n h}\|x[k]\| .
\end{aligned}
$$

If we let

$$
\gamma_{\nu}:=\sum_{i=1}^{n-1} i^{n},
$$

then we can easily determine the upper bound on the norm of $\mathcal{V}_{k}$ :

$$
\left\|\mathcal{V}_{k}\right\| \leq \gamma_{\nu} \frac{h^{n}}{n !}\|C\| c_{a}^{n} e^{c_{a} n h}\|x[k]\|
$$

We can utilize (4.21) and (4.23) in (4.20) to obtain a bound on state estimation error:

$$
\begin{aligned}
\|\hat{x}[k]-x[k]\| & \leq\left\|\mathcal{O}^{-1}(C, A(\alpha))\right\|\left\|S_{n}^{-1}\right\| \frac{(n-1) !}{h^{n-1}} \gamma_{\nu} \frac{h^{n}}{n !}\|C\| c_{a}^{n} e^{c_{a} n h}\|x[k]\| \\
& =\left\|\mathcal{O}^{-1}(C, A(\alpha))\right\|\left\|S_{n}^{-1}\right\|\|C\| c_{a}^{n} e^{c_{a} n h} \gamma_{\nu} \frac{1}{n}\|x[k]\| h .
\end{aligned}
$$


It is easy to see that there exists a constant $\gamma_{x}$ such that for small $h$ we have

$$
\|\hat{x}[k]-x[k]\| \leq \gamma_{x}\|x[k]\| h .
$$

Hence, as long as the size of plant's state remains bounded, the state estimation error is also bounded and becomes smaller as the sampling period approaches zero.

\subsection{Parameter Estimation}

Our controller design requires the parameter estimate to evaluate the gain matrices $F(\hat{\alpha}[k])$, $G(\hat{\alpha}[k]), H(\hat{\alpha}[k])$, and $K(\hat{\alpha}[k])$. However, since the values of $\alpha$ are not generally measurable, we are required to estimate the value of the parameter in every period. If $\alpha(t)$ has a jump on $[k T,(k+1) T)$, we would expect that it would be difficult, if not impossible, to estimate $\alpha(k T)$. So from this point on, we assume that $\alpha \in A C(\mathcal{A})$. We will pick some $\rho>0$ and apply a probing signal $u_{p}[k]=\rho(\|\hat{x}[k]\|+\|z[k]\|)$ for $T_{2}=(\bar{n}+2) h$ seconds. If we let $\bar{t}_{1}:=k T+n h$, then we can write

$$
u_{p}[k]=\rho(\|\hat{x}[k]\|+\|z[k]\|) \quad \text { for } \quad t \in\left[\bar{t}_{1}, \bar{t}_{1}+(\bar{n}+2) h\right) .
$$

We will employ a similar estimation procedure as in the previous section. To begin, consider the first $\bar{n}+1$ derivatives of $y(t)$ for $t \in\left(\bar{t}_{1}, \bar{t}_{1}+(\bar{n}+2) h\right)$ :

$$
\begin{aligned}
\dot{y}(t) & =C A(\alpha(t)) x(t)+C B(\alpha(t)) u_{p}[k], \\
\ddot{y}(t) & =C A^{2}(\alpha(t)) x(t)+C A B(\alpha(t)) u_{p}[k], \\
& \vdots \\
y^{(\bar{n}+1)}(t) & =C A^{\bar{n}+1}(\alpha(t)) x(t)+\underbrace{C A^{\bar{n}} B(\alpha(t))}_{f(\alpha(t))} u_{p}[k] .
\end{aligned}
$$

Since $C A^{\bar{n}+1}(\alpha(t))$ is constant by Assumption 3, we will denote it simply as $C A^{\bar{n}+1}$. By integrating $y^{(\bar{n}+1)}(t)$ we obtain:

$$
\begin{aligned}
y^{(\bar{n})}(t) & =y^{(\bar{n})}\left(\bar{t}_{1}^{+}\right)+\int_{\bar{t}_{1}}^{t} y^{(\bar{n}+1)}(\tau) d \tau \\
& =y^{(\bar{n})}\left(\bar{t}_{1}^{+}\right)+\int_{\bar{t}_{1}}^{t} C A^{\bar{n}+1} x(\tau) d \tau+\int_{\bar{t}_{1}}^{t} f(\alpha(\tau)) u_{p}[k] d \tau \\
& =y^{(\bar{n})}\left(\bar{t}_{1}^{+}\right)+C A^{\bar{n}+1} \int_{\bar{t}_{1}}^{t} x(\tau) d \tau+u_{p}[k] \int_{\bar{t}_{1}}^{t} f(\alpha(\tau)) d \tau .
\end{aligned}
$$


We can get an expression for $x(\tau)$ by integrating equation (3.26):

$$
x(\tau)=x\left(\bar{t}_{1}\right)+\int_{\bar{t}_{1}}^{\tau} A(\alpha(\theta)) x(\theta) d \theta+\int_{\bar{t}_{1}}^{\tau} B(\alpha(\theta)) u_{p}[k] d \theta .
$$

Also, the expression for $f(\alpha(\tau))$ is obtained in a similar fashion:

$$
f(\alpha(\tau))=f\left(\alpha\left(\bar{t}_{1}\right)\right)+\int_{\bar{t}_{1}}^{\tau} \frac{d f(\alpha(\theta))}{d \theta} d \theta .
$$

Using the expressions for $x(\tau)$ and $f(\alpha(\tau))$ in (4.27) results in

$$
\begin{aligned}
y^{(\bar{n})}(t)= & y^{(\bar{n})}\left(\bar{t}_{1}^{+}\right)+C A^{\bar{n}+1}\left(\int_{\bar{t}_{1}}^{t} x\left(\bar{t}_{1}\right) d \tau+\int_{\bar{t}_{1}}^{t} \int_{\bar{t}_{1}}^{\tau} A(\alpha(\theta)) x(\theta) d \theta d \tau\right. \\
& \left.+u_{p}[k] \int_{\bar{t}_{1}}^{t} \int_{\bar{t}_{1}}^{\tau} B(\alpha(\theta)) d \theta d \tau\right)+u_{p}[k]\left(\int_{\bar{t}_{1}}^{t} f\left(\alpha\left(\bar{t}_{1}\right)\right) d \tau+\int_{\bar{t}_{1}}^{t} \int_{\bar{t}_{1}}^{\tau} \frac{d f(\alpha(\theta))}{d \theta} d \theta d \tau\right) .
\end{aligned}
$$

We will define $\mu_{1}(t)$ as

$$
\begin{aligned}
\mu_{1}(t):= & C A^{\bar{n}+1}\left(\int_{\bar{t}_{1}}^{t} \int_{\bar{t}_{1}}^{\tau} A(\alpha(\theta)) x(\theta) d \theta d \tau+u_{p}[k] \int_{\bar{t}_{1}}^{t} \int_{\bar{t}_{1}}^{\tau} B(\alpha(\theta)) d \theta d \tau\right) \\
& +u_{p}[k] \int_{\bar{t}_{1}}^{t} \int_{\bar{t}_{1}}^{\tau} \frac{d f(\alpha(\theta))}{d \theta} d \theta d \tau,
\end{aligned}
$$

yielding the following

$$
\begin{aligned}
y^{(\bar{n})}(t) & =y^{(\bar{n})}\left(\bar{t}_{1}^{+}\right)+C A^{\bar{n}+1} x\left(\bar{t}_{1}\right)\left(t-\bar{t}_{1}\right)+u_{p}[k] f\left(\alpha\left(\bar{t}_{1}\right)\right)\left(t-\bar{t}_{1}\right)+\mu_{1}(t) \\
& =y^{(\bar{n})}\left(\bar{t}_{1}^{+}\right)+\left(t-\bar{t}_{1}\right) y^{(\bar{n}+1)}\left(\bar{t}_{1}^{+}\right)+\mu_{1}(t) .
\end{aligned}
$$

We would also like to derive a bound on $\left|\mu_{1}(t)\right|$ :

$$
\begin{aligned}
\left|\mu_{1}(t)\right| \leq & \left\|C A^{\bar{n}+1}\right\|\left(\int_{\bar{t}_{1}}^{t} \int_{\bar{t}_{1}}^{\tau}\|A(\alpha(\theta))\|\|x(\theta)\| d \theta d \tau+\left|u_{p}[k]\right| \int_{\bar{t}_{1}}^{t} \int_{\bar{t}_{1}}^{\tau}\|B(\alpha(\theta))\| d \theta d \tau\right) \\
& +\left|u_{p}[k]\right| \int_{\bar{t}_{1}}^{t} \int_{\bar{t}_{1}}^{\tau}\left|\frac{d f(\alpha(\theta))}{d \theta}\right| d \theta d \tau \\
\leq & \left\|C A^{\bar{n}+1}\right\|\left(c_{a} \int_{\bar{t}_{1}}^{t} \int_{\bar{t}_{1}}^{\tau}\|x(\theta)\| d \theta d \tau+\left|u_{p}[k]\right| c_{b} \frac{\left(t-\bar{t}_{1}\right)^{2}}{2}\right)+\left|u_{p}[k]\right| \delta_{f} \delta_{\alpha} \frac{\left(t-\bar{t}_{1}\right)^{2}}{2} .
\end{aligned}
$$

We will apply Lemma 1 twice to get a bound on $\|x(\theta)\|$ :

$$
\begin{aligned}
\|x(\theta)\| & \leq\left[\left\|x\left(\bar{t}_{1}\right)\right\|+\left(\theta-\bar{t}_{1}\right)\left|u_{p}[k]\right| c_{b}\right] e^{c_{a}\left(\theta-\bar{t}_{1}\right)} \\
& \leq\left[\left\|x\left(\bar{t}_{1}\right)\right\|+(\bar{n}+2) h\left|u_{p}[k]\right| c_{b}\right] e^{c_{a}(\bar{n}+2) h} \\
& \leq\left[\|x(k T)\| e^{c_{a} n h}+(\bar{n}+2) h\left|u_{p}[k]\right| c_{b}\right] e^{c_{a}(\bar{n}+2) h} \\
& =e^{c_{a}(n+\bar{n}+2) h}\|x[k]\|+(\bar{n}+2) h c_{b} e^{c_{a}(\bar{n}+2) h}\left|u_{p}[k]\right|, \quad t \in\left(\bar{t}_{1}, \bar{t}_{1}+(\bar{n}+2) h\right) .
\end{aligned}
$$


Finally,

$$
\begin{aligned}
\left|\mu_{1}(t)\right| \leq & \left\|C A^{\bar{n}+1}\right\| c_{a} e^{c_{a}(n+\bar{n}+2) h} \frac{\left(t-\bar{t}_{1}\right)^{2}}{2}\|x[k]\|+\left[\left\|C A^{\bar{n}+1}\right\| c_{a}(\bar{n}+2) h c_{b} e^{c_{a}(\bar{n}+2) h}\right. \\
& \left.+\left\|C A^{\bar{n}+1}\right\| c_{b}+\delta_{f} \delta_{\alpha}\right] \frac{\left(t-\bar{t}_{1}\right)^{2}}{2}\left|u_{p}[k]\right|, \quad t \in\left(\bar{t}_{1}, \bar{t}_{1}+(\bar{n}+2) h\right) .
\end{aligned}
$$

Hence, there exist constants $\gamma_{1}$ and $\gamma_{2}$ such that for small $h$

$$
\left|\mu_{1}(t)\right| \leq\left(\gamma_{1}\|x[k]\|+\gamma_{2}\left|u_{p}[k]\right|\right) \frac{\left(t-\bar{t}_{1}\right)^{2}}{2}, \quad t \in\left(\bar{t}_{1}, \bar{t}_{1}+(\bar{n}+2) h\right) .
$$

Integrating $y^{(\bar{n})}(t)$ we obtain

$$
\begin{aligned}
y^{(\bar{n}-1)}(t) & =y^{(\bar{n}-1)}\left(\bar{t}_{1}^{+}\right)+\int_{\bar{t}_{1}}^{t} y^{(\bar{n})}(\tau) d \tau \\
& =y^{(\bar{n}-1)}\left(\bar{t}_{1}^{+}\right)+\left(t-\bar{t}_{1}\right) y^{(\bar{n})}\left(\bar{t}_{1}^{+}\right)+\frac{\left(t-\bar{t}_{1}\right)^{2}}{2} y^{(\bar{n}+1)}\left(\bar{t}_{1}^{+}\right)+\underbrace{\int_{\bar{t}_{1}}^{t} \mu_{1}(\tau) d \tau}_{=: \mu_{2}(t)},
\end{aligned}
$$

where

$$
\begin{aligned}
\left|\mu_{2}(t)\right| & \leq \int_{\bar{t}_{1}}^{t}\left|\mu_{1}(\tau)\right| d \tau \\
& \leq\left(\gamma_{1}\|x[k]\|+\gamma_{2}\left|u_{p}[k]\right|\right) \frac{\left(t-\bar{t}_{1}\right)^{3}}{3 !}, \quad t \in\left(\bar{t}_{1}, \bar{t}_{1}+(\bar{n}+2) h\right) .
\end{aligned}
$$

It is now easy to recognize the pattern for the expansion of $y(t)$ : it is of the form

$$
\begin{aligned}
y(t)= & y\left(\bar{t}_{1}\right)+\left(t-\bar{t}_{1}\right) \dot{y}\left(\bar{t}_{1}^{+}\right)+\frac{\left(t-\bar{t}_{1}\right)^{2}}{2 !} \ddot{y}\left(\bar{t}_{1}^{+}\right)+\cdots+\frac{\left(t-\bar{t}_{1}\right)^{\bar{n}+1}}{(\bar{n}+1) !} y^{(\bar{n}+1)}\left(\bar{t}_{1}^{+}\right) \\
& +\mu_{\bar{n}+1}(t), \quad t \in\left(\bar{t}_{1}, \bar{t}_{1}+(\bar{n}+2) h\right),
\end{aligned}
$$

with

$$
\left|\mu_{\bar{n}+1}(t)\right| \leq\left(\gamma_{1}\|x[k]\|+\gamma_{2}\left|u_{p}[k]\right|\right) \frac{\left(t-\bar{t}_{1}\right)^{(\bar{n}+2)}}{(\bar{n}+2) !}, \quad t \in\left(\bar{t}_{1}, \bar{t}_{1}+(\bar{n}+2) h\right) .
$$

We will define two vectors of sampled values:

$$
\overline{\mathcal{Y}}_{k}:=\left[\begin{array}{c}
y\left(\bar{t}_{1}\right) \\
y\left(\bar{t}_{1}+h\right) \\
\vdots \\
y\left(\bar{t}_{1}+(\bar{n}+1) h\right)
\end{array}\right] \text { and } \mathcal{M}_{k}:=\left[\begin{array}{c}
0 \\
\mu_{\bar{n}+1}\left(\bar{t}_{1}+h\right) \\
\vdots \\
\mu_{\bar{n}+1}\left(\bar{t}_{1}+(\bar{n}+1) h\right)
\end{array}\right] .
$$


Using these vectors we can write

$$
\begin{aligned}
& \overline{\mathcal{Y}}_{k}=\left[\begin{array}{c}
y\left(\bar{t}_{1}\right) \\
y\left(\bar{t}_{1}\right)+h \dot{y}\left(\bar{t}_{1}^{+}\right)+\frac{h^{2}}{2 !} \ddot{y}\left(\bar{t}_{1}^{+}\right)+\ldots+\frac{h^{\bar{n}+1}}{(\bar{n}+1) !} y^{(\bar{n}+1)}\left(\bar{t}_{1}^{+}\right) \\
y\left(\bar{t}_{1}\right)+2 h \dot{y}\left(\bar{t}_{1}^{+}\right)+\frac{4 h^{2}}{2 !} \ddot{y}\left(\bar{t}_{1}^{+}\right)+\ldots+\frac{2^{\bar{n}+1} h^{\bar{n}+1}}{(\bar{n}+1) !} y^{(\bar{n}+1)}\left(\bar{t}_{1}^{+}\right) \\
\vdots \\
y\left(\bar{t}_{1}\right)+(\bar{n}+1) h \dot{y}\left(\bar{t}_{1}^{+}\right)+\frac{(\bar{n}+1)^{2} h^{2}}{2 !} \ddot{y}\left(\bar{t}_{1}^{+}\right)+\ldots+\frac{(\bar{n}+1)^{\bar{n}+1} h^{\bar{n}+1}}{\bar{n}+1 !} y^{(\bar{n}+1)\left(\bar{t}_{1}^{+}\right)}
\end{array}\right]+\mathcal{M}_{k} \\
& =\left[\begin{array}{ccccc}
1 & 0 & 0 & \cdots & 0 \\
1 & h & \frac{h^{2}}{2 !} & \cdots & \frac{h^{\bar{n}+1}}{(\bar{n}+1) !} \\
1 & 2 h & \frac{4 h^{2}}{2 !} & \cdots & \frac{2^{\bar{n}+1} h^{\bar{n}+1}}{(\bar{n}+1) !} \\
\vdots & \vdots & \vdots & \ddots & \vdots \\
1 & (\bar{n}+1) h & \frac{(\bar{n}+1)^{2} h^{2}}{2 !} & \cdots & \frac{(\bar{n}+1)^{\bar{n}+1} h^{\bar{n}+1}}{(\bar{n}+1) !}
\end{array}\right]\left[\begin{array}{c}
y\left(\bar{t}_{1}\right) \\
\dot{y}\left(\bar{t}_{1}^{+}\right) \\
\ddot{y}\left(\bar{t}_{1}^{+}\right) \\
\vdots \\
y^{(\bar{n}+1)}\left(\bar{t}_{1}^{+}\right)
\end{array}\right]+\mathcal{M}_{k} \\
& =S_{\bar{n}+2} Q_{\bar{n}+2}(h)\left[\begin{array}{c}
y\left(\bar{t}_{1}\right) \\
\dot{y}\left(\bar{t}_{1}^{+}\right) \\
\vdots \\
y^{(\bar{n}+1)}\left(\bar{t}_{1}^{+}\right)
\end{array}\right]+\mathcal{M}_{k} \text {, }
\end{aligned}
$$

where $S_{\bar{n}+2}$ and $Q_{\bar{n}+2}(h)$ are as defined in (4.15) and (4.16). Recalling the observations from Section 4.1 concerning the existence of $S_{\bar{n}+2}^{-1}$ and $Q_{\bar{n}+2}^{-1}(h)$, the above equation transforms into

$$
\left[\begin{array}{c}
y\left(\bar{t}_{1}\right) \\
\dot{y}\left(\bar{t}_{1}^{+}\right) \\
\vdots \\
y^{(\bar{n}+1)}\left(\bar{t}_{1}^{+}\right)
\end{array}\right]=Q_{\bar{n}+2}^{-1}(h) S_{\bar{n}+2}^{-1}\left(\overline{\mathcal{Y}}_{k}-\mathcal{M}_{k}\right) .
$$

If we define a $1 \times(\bar{n}+2)$ matrix $E:=\left[\begin{array}{llll}0 & \ldots & 0 & 1\end{array}\right]$, we can explicitly determine $y^{(\bar{n}+1)}\left(\bar{t}_{1}\right)$ by multiplying both sides of equation $(4.32)$ by $E$ on the left:

$$
y^{(\bar{n}+1)}\left(\bar{t}_{1}^{+}\right)=E Q_{\bar{n}+2}^{-1}(h) S_{\bar{n}+2}^{-1}\left(\overline{\mathcal{Y}}_{k}-\mathcal{M}_{k}\right) .
$$

Using equation (4.26) we can calculate

$$
f\left(\alpha\left(\bar{t}_{1}\right)\right)=\frac{1}{u_{p}[k]}\left[E Q_{\bar{n}+2}^{-1}(h) S_{\bar{n}+2}^{-1}\left(\overline{\mathcal{Y}}_{k}-\mathcal{M}_{k}\right)-C A^{\bar{n}+1} x\left(\bar{t}_{1}\right)\right] .
$$


We will introduce an auxiliary variable $\breve{f}[k]$ representing a crude estimate of $f\left(\alpha\left(\bar{t}_{1}\right)\right)$ :

$$
\check{f}[k]:=\left\{\begin{array}{cc}
\frac{1}{u_{p}[k]}\left[E Q_{\bar{n}+2}^{-1}(h) S_{\bar{n}+2}^{-1} \overline{\mathcal{Y}}_{k}-C A^{\bar{n}+1} \hat{x}[k]\right] . & \text { if } u_{p}[k] \neq 0 ; \\
f(\underline{\alpha}) & \text { if } u_{p}[k]=0 .
\end{array}\right.
$$

We would like to obtain an estimate of $f\left(\alpha\left(\bar{t}_{1}\right)\right)$ that will always take values in $\mathcal{F}$. This can be achieved by projecting $\check{f}[k]$ onto $\mathcal{F}$. Hence, we define another estimate variable, $\hat{f}[k]$ :

$$
\hat{f}[k]:=\Pi_{\mathcal{F}}(\check{f}[k]) .
$$

Note that with this definition of $\hat{f}[k]$, we have that $f^{-1}(\hat{f}[k]) \in \mathcal{A}$. On that basis, we can calculate the estimate of $\alpha\left(\bar{t}_{1}\right)$ that will always belong to $\mathcal{A}$ :

$$
\hat{\alpha}[k]:=f^{-1}(\hat{f}[k]) .
$$

It must be noted when $u_{p}[k]=0$, we have $\hat{\alpha}[k]=\underline{\alpha}$, which is not an accurate estimate of $\alpha\left(\bar{t}_{1}\right)$ in most cases. However, this case is trivial since it can only happen when $\hat{x}[k]=0$ and $z[k]=0$. Consider the size of $x[k]$ in this case: for small $h$,

$$
\begin{aligned}
\|x[k]\| & \leq\|x[k]-\hat{x}[k]\|+\|\hat{x}[k]\| \\
& \leq \gamma_{x} h\|x[k]\|+\|\hat{x}[k]\|,
\end{aligned}
$$

so for $h<\frac{1}{\gamma_{x}}$, we have

$$
\|x[k]\| \leq \frac{1}{1-\gamma_{x} h}\|\hat{x}[k]\| .
$$

Hence, for small $h$, if $\hat{x}[k]=0$, then $x[k]=0$. On the other hand, from (4.24) we see that for small $h$, if $x[k]=0$, then $\hat{x}[k]=0$. We conclude that for small $h,\left[\begin{array}{l}\hat{x}[k] \\ z[k]\end{array}\right]=0$ if and only if $\left[\begin{array}{l}x[k] \\ z[k]\end{array}\right]=0$. Hence, for small $h$, when $u_{p}[k]=0$, the plant and controller states are already at the origin, so a potentially inaccurate estimate of the parameter value is not crucial. Hence, for the rest of the section we will assume that $\left[\begin{array}{l}x[k] \\ z[k]\end{array}\right] \neq 0$.

Our goal is to derive an upper bound on $\left|\hat{f}[k]-f\left(\alpha\left(\bar{t}_{1}\right)\right)\right|$. We will begin by defining

$$
\tilde{f}[k]:=\check{f}[k]-f\left(\alpha\left(\bar{t}_{1}\right)\right) .
$$


Let us consider $|\tilde{f}[k]|$ : using (4.33) and (4.34) yields

$$
|\tilde{f}[k]| \leq \frac{1}{\left|u_{p}[k]\right|}\left[\left\|C A^{\bar{n}+1}\right\|\left\|\hat{x}[k]-x\left(\bar{t}_{1}\right)\right\|+\|E\|\left\|Q_{\bar{n}+2}^{-1}(h)\right\|\left\|S_{\bar{n}+2}^{-1}\right\|\left\|\mathcal{M}_{k}\right\|\right] .
$$

We will first examine $\left\|\hat{x}[k]-x\left(\bar{t}_{1}\right)\right\|$ for small $h$ :

$$
\begin{aligned}
\left\|\hat{x}[k]-x\left(\bar{t}_{1}\right)\right\| & \left.=\| \hat{x}[k]-\left[x(k T)+\int_{k T}^{k T+n h} \dot{x}(\tau) d \tau\right)\right] \| \\
& \leq\|\hat{x}[k]-x[k]\|+\int_{k T}^{k T+n h} \| A(\alpha(\tau)\|\| x(\tau) \| d \tau \\
& \leq \gamma_{x}\|x[k]\| h+c_{a} \int_{k T}^{k T+n h}\|x(k T)\| e^{c_{a}(\tau-k T)} d \tau \\
& \leq \gamma_{x}\|x[k]\| h+c_{a}\|x[k]\| e^{c_{a} n h} n h \\
& =\left(\gamma_{x}+n c_{a} e^{c_{a} n h}\right)\|x[k]\| h .
\end{aligned}
$$

To derive an upper bound on $\left\|\mathcal{M}_{k}\right\|$, we will first examine the bounds on the individual elements using (4.30):

$$
\begin{aligned}
\left|\mu_{\bar{n}+1}\left(\bar{t}_{1}+h\right)\right| & \leq\left(\gamma_{1}\|x[k]\|+\gamma_{2}\left|u_{p}[k]\right|\right) \frac{h^{\bar{n}+2}}{(\bar{n}+2) !}, \\
\left|\mu_{\bar{n}+1}\left(\bar{t}_{1}+2 h\right)\right| & \leq\left(\gamma_{1}\|x[k]\|+\gamma_{2}\left|u_{p}[k]\right|\right) \frac{2^{(\bar{n}+2)} h^{\bar{n}+2}}{(\bar{n}+2) !}, \\
& \vdots \\
\left|\mu_{\bar{n}+1}\left(\bar{t}_{1}+(\bar{n}+1) h\right)\right| & \leq\left(\gamma_{1}\|x[k]\|+\gamma_{2}\left|u_{p}[k]\right|\right) \frac{(\bar{n}+1)^{(\bar{n}+2)} h^{\bar{n}+2}}{(\bar{n}+2) !} .
\end{aligned}
$$

By letting

$$
\gamma_{\mu}:=\sum_{i=1}^{\bar{n}+1} i^{\bar{n}+2}
$$

we can write

$$
\left\|\mathcal{M}_{k}\right\| \leq \gamma_{\mu}\left[\gamma_{1}\|x[k]\|+\gamma_{2}\left|u_{p}[k]\right|\right] \frac{h^{\bar{n}+2}}{(\bar{n}+2) !}
$$

Lastly, it is easy to see that

$$
\|E\|=1 \quad \text { and } \quad\left\|Q_{\bar{n}+2}^{-1}(h)\right\|=\frac{(\bar{n}+1) !}{h^{\bar{n}+1}} .
$$


Now we can use these intermediate results in (4.39):

$$
\begin{aligned}
|\tilde{f}[k]| \leq & \frac{1}{\left|u_{p}[k]\right|}\left[\left\|C A^{\bar{n}+1}\right\|\left(\gamma_{x}+n c_{a} e^{c_{a} n h}\right)\|x[k]\| h\right. \\
& \left.+\left\|S_{\bar{n}+2}^{-1}\right\| \frac{(\bar{n}+1) !}{h^{\bar{n}+1}} \gamma_{\mu}\left[\gamma_{1}\|x[k]\|+\gamma_{2}\left|u_{p}[k]\right|\right] \frac{h^{\bar{n}+2}}{(\bar{n}+2) !}\right] .
\end{aligned}
$$

We will use (4.37) to bound $\|x[k]\|$, so it follows that there exists a constant $\gamma_{f}$ such that for small $h$ :

$$
\begin{aligned}
|\tilde{f}[k]| & \leq \gamma_{f} \frac{\rho}{1+\rho} \frac{\|\hat{x}[k]\|+\left|u_{p}[k]\right|}{\left|u_{p}[k]\right|} h \\
& =\gamma_{f} \frac{\rho}{1+\rho} \frac{\|\hat{x}[k]\|+\rho(\hat{x}[k]+\|z[k]\|)}{\rho(\|\hat{x}[k]\|+\|z[k]\|)} h \\
& \leq \gamma_{f} h .
\end{aligned}
$$

In order to obtain an accurate estimate of the parameter, we need to ensure that $\check{f}[k]$ is always projected onto the correct interval, i.e. $\hat{f}[k]$ and $f\left(\bar{t}_{1}\right)$ belong to the same interval in $\mathcal{F}$. This is achieved if $h$ is chosen small enough such that $|\tilde{f}[k]|$ is less than half the smallest distance between adjacent intervals in $\mathcal{F}$. Let us define $d^{*}$ as

$$
d^{*}:=\min _{j}\left(\underline{f}_{j+1}-\bar{f}_{j}\right) \quad \text { for } j=1,2, \ldots, q-1,
$$

so if we choose $h<\frac{d^{*}}{2 \gamma_{f}}$, then by construction, $\hat{f}[k]$ always belongs to the same interval in $\mathcal{F}$ as $f\left(\alpha\left(\bar{t}_{1}\right)\right)$. We conclude that for small $h$, we have

$$
\begin{aligned}
\left|\hat{f}[k]-f\left(\alpha\left(\bar{t}_{1}\right)\right)\right| & \leq|\tilde{f}[k]| \\
& \leq \gamma_{f} h .
\end{aligned}
$$

Defining $\alpha[k]:=\alpha(k T)$, we can use (4.46) to derive an upper bound on the parameter estimation error when $\left\|\left[\begin{array}{l}x[k] \\ z[k]\end{array}\right]\right\|>0$ - for small $h$ :

$$
\begin{aligned}
\hat{\alpha}[k] & =f^{-1}(\hat{f}[k]) \\
& =\alpha\left(\bar{t}_{1}\right)+\int_{f\left(\alpha\left(\bar{t}_{1}\right)\right)}^{\hat{f}[k]} \frac{d f^{-1}(\phi)}{d \phi} d \phi \\
& =\alpha(k T)+\int_{k T}^{\bar{t}_{1}} \dot{\alpha}(\tau) d \tau+\int_{f\left(\alpha\left(\bar{t}_{1}\right)\right)}^{\hat{f}[k]} \frac{d f^{-1}(\phi)}{d \phi} d \phi .
\end{aligned}
$$


By Assumption 4 ii) we obtain a bound on $\left|\frac{d f^{-1}(\phi)}{d \phi}\right|$ :

$$
\operatorname{ess} \inf _{\alpha \in \mathcal{A}}\left|\frac{d f(\alpha)}{d \alpha}\right|>\delta_{i} \Longleftrightarrow \operatorname{ess} \sup _{\phi \in \mathcal{F}}\left|\frac{d f^{-1}(\phi)}{d \phi}\right|<\frac{1}{\delta_{i}}
$$

In addition, we will use Assumption 5 to bound $|\dot{\alpha}(\tau)|$. From here we get the desired result - for small $h$ :

$$
\begin{aligned}
|\hat{\alpha}[k]-\alpha[k]| & \leq \int_{k T}^{\bar{t}_{1}}|\dot{\alpha}(\tau)| d \tau+\int_{f\left(\alpha\left(\bar{t}_{1}\right)\right)}^{\hat{f}[k]}\left|\frac{d f^{-1}(\phi)}{d \phi}\right| d \phi \\
& \leq \delta_{\alpha}\left(\bar{t}_{1}-k T\right)+\frac{1}{\delta_{i}}\left|\hat{f}[k]-f\left(\alpha\left(\bar{t}_{1}\right)\right)\right| \\
& \leq \delta_{\alpha} T_{1}+\frac{\gamma_{f}}{\delta_{i}} h \\
& =\left(n \delta_{\alpha}+\frac{\gamma_{f}}{\delta_{i}}\right) h .
\end{aligned}
$$

\subsection{Discrete-Time Stability Analysis}

In previous sections we established that we can obtain a simple bound on the error between the estimate of the state and the actual value. The state estimate becomes better as the sampling period become smaller. For much of this section, we will assume that the parameter estimation error in the period $[k T,(k+1) T)$ is small, i.e. it is bounded by (4.49), which is the case if $h$ is small and $\left\|\left[\begin{array}{l}x[k] \\ z[k]\end{array}\right]\right\|>0$. Hence, from now on and unless otherwise stated, we will assume that $k \in \mathbb{Z}^{+}$satisfies $\left\|\left[\begin{array}{l}x[k] \\ z[k]\end{array}\right]\right\|>0$. With these results we can now move on to analyze the stability of the closed loop system. The stabilizing control signal $u_{s}[k]$ will be applied for the last $T_{3}$ seconds in every period. We will use equations (4.2) and (4.3) to calculate $u_{s}[k]$.

Our goal is to discretize the closed loop system with a sampling period $T$. We will start by modifying equations (4.2) and (4.3) to use $x[k]$ and $\alpha[k]$ in place of the estimate variables. To this end, from (4.24) it is easy to see that for small $h$ the following is true:

$$
\hat{x}[k]=x[k]+\epsilon_{x}[k], \quad \text { where }\left\|\epsilon_{x}[k]\right\| \leq \gamma_{x}\|x[k]\| h .
$$


Firstly, consider equation (4.2) - for small $h$ we have

$$
\begin{aligned}
z[k+1]= & {[I+T F(\hat{\alpha}[k])] z[k]+T G(\hat{\alpha}[k]) \hat{x}[k] } \\
= & {\left[I+T\left(F(\alpha[k])+\int_{\alpha[k]}^{\hat{\alpha}[k]} \frac{d F(a)}{d a} d a\right)\right] z[k] } \\
& +T\left(G(\alpha[k])+\int_{\alpha[k]}^{\hat{\alpha}[k]} \frac{d G(a)}{d a} d a\right) x[k]+T G(\hat{\alpha}[k]) \epsilon_{x}[k] \\
= & z[k]+T G(\alpha[k]) x[k]+T F(\alpha[k]) z[k] \\
& +T\left[\int_{\alpha[k]}^{\hat{\alpha}[k]} \frac{d F(a)}{d a} d a z[k]+\int_{\alpha[k]}^{\hat{\alpha}[k]} \frac{d G(a)}{d a} d a x[k]+G(\hat{\alpha}[k]) \epsilon_{x}[k]\right] .
\end{aligned}
$$

If we define

$$
e_{1}[k]:=T\left[\int_{\alpha[k]}^{\hat{\alpha}[k]} \frac{d F(a)}{d a} d a z[k]+\int_{\alpha[k]}^{\hat{\alpha}[k]} \frac{d G(a)}{d a} d a x[k]+G(\hat{\alpha}[k]) \epsilon_{x}[k]\right],
$$

then we can write

$$
z[k+1]=z[k]+T G(\alpha[k]) x[k]+T F(\alpha[k]) z[k]+e_{1}[k] .
$$

We are interested in deriving an upper bound on $\left\|e_{1}[k]\right\|$ :

$$
\begin{aligned}
\left\|e_{1}[k]\right\| & \leq T\left[\int_{\alpha[k]}^{\hat{\alpha}[k]}\left\|\frac{d F(a)}{d a}\right\| d a\|z[k]\|+\int_{\alpha[k]}^{\hat{\alpha}[k]}\left\|\frac{d G(a)}{d a}\right\| d a\|x[k]\|+\|G(\hat{\alpha}[k])\|\left\|\epsilon_{x}[k]\right\|\right] \\
& \leq T\left[\left(\delta_{F}\|z[k]\|+\delta_{G}\|x[k]\|\right)|\hat{\alpha}[k]-\alpha[k]|+c_{g}\left\|\epsilon_{x}[k]\right\|\right] \\
& \leq T\left[\left(\delta_{F}\|z[k]\|+\delta_{G}\|x[k]\|\right)\left(n \delta_{\alpha}+\frac{\gamma_{f}}{\delta_{i}}\right) h+c_{g} \gamma_{x}\|x[k]\| h\right] .
\end{aligned}
$$

Recalling that $h=T / N$, for small $h$ we obtain

$$
\left\|e_{1}[k]\right\| \leq\left[\frac{\delta_{G}}{N}\left(n \delta_{\alpha}+\frac{\gamma_{f}}{\delta_{i}}\right)+\frac{c_{g} \gamma_{x}}{N}\right] T^{2}\|x[k]\|+\frac{\delta_{F}}{N}\left(n \delta_{\alpha}+\frac{\gamma_{f}}{\delta_{i}}\right) T^{2}\|z[k]\| .
$$

It is easy to see that there exist constants $\gamma_{3}$ and $\gamma_{4}$ such that for small $h$

$$
\left\|e_{1}[k]\right\| \leq\left(\gamma_{3}\|x[k]\|+\gamma_{4}\|z[k]\|\right) T^{2} .
$$


Now let us consider equation (4.3) - for small $h$ :

$$
\begin{aligned}
u_{s}[k]= & \frac{T}{T_{3}}[H(\hat{\alpha}[k]) z[k]+K(\hat{\alpha}[k]) \hat{x}[k]]-\frac{T_{2}}{T_{3}} u_{p}[k] \\
= & \frac{T}{T_{3}}\left[\left(H(\alpha[k])+\int_{\alpha[k]}^{\hat{\alpha}[k]} \frac{d H(a)}{d a} d a\right) z[k]\right. \\
& \left.+\left(K(\alpha[k])+\int_{\alpha[k]}^{\hat{\alpha}[k]} \frac{d K(a)}{d a} d a\right) x[k]+K(\hat{\alpha}[k]) \epsilon_{x}[k]\right]-\frac{T_{2}}{T_{3}} u_{p}[k] \\
= & \frac{T}{T_{3}}[H(\alpha[k]) z[k]+K(\alpha[k]) x[k]]-\frac{T_{2}}{T_{3}} u_{p}[k] \\
& +\frac{T}{T_{3}}\left[\int_{\alpha[k]}^{\hat{\alpha}[k]} \frac{d H(a)}{d a} d a z[k]+\int_{\alpha[k]}^{\hat{\alpha}[k]} \frac{d K(a)}{d a} d a x[k]+K(\hat{\alpha}[k]) \epsilon_{x}[k]\right] .
\end{aligned}
$$

Letting

$$
e_{2}[k]:=T\left[\int_{\alpha[k]}^{\hat{\alpha}[k]} \frac{d H(a)}{d a} d a z[k]+\int_{\alpha[k]}^{\hat{\alpha}[k]} \frac{d K(a)}{d a} d a x[k]+K(\hat{\alpha}[k]) \epsilon_{x}[k]\right],
$$

we obtain

$$
u_{s}[k]=\frac{T}{T_{3}}[H(\alpha[k]) z[k]+K(\alpha[k]) x[k]]-\frac{T_{2}}{T_{3}} u_{p}[k]+\frac{1}{T_{3}} e_{2}[k],
$$

where for small $h$

$$
\begin{aligned}
\left\|e_{2}[k]\right\| & \leq T\left[\int_{\alpha[k]}^{\hat{\alpha}[k]}\left\|\frac{d H(a)}{d a}\right\| d a\|z[k]\|+\int_{\alpha[k]}^{\hat{\alpha}[k]}\left\|\frac{d K(a)}{d a}\right\| d a\|x[k]\|+\|K(\hat{\alpha}[k])\|\left\|\epsilon_{x}[k]\right\|\right] \\
& \leq T\left[\left(\delta_{H}\|z[k]\|+\delta_{K}\|x[k]\|\right)|\hat{\alpha}[k]-\alpha[k]|+c_{k}\left\|\epsilon_{x}[k]\right\|\right] \\
& \leq T\left[\left(\delta_{H}\|z[k]\|+\delta_{K}\|x[k]\|\right)\left(n \delta_{\alpha}+\frac{\gamma_{f}}{\delta_{i}}\right) h+c_{k} \gamma_{x}\|x[k]\| h\right] \\
& \leq\left[\frac{\delta_{K}}{N}\left(n \delta_{\alpha}+\frac{\gamma_{f}}{\delta_{i}}\right)+\frac{c_{k} \gamma_{x}}{N}\right] T^{2}\|x[k]\|+\frac{\delta_{H}}{N}\left(n \delta_{\alpha}+\frac{\gamma_{f}}{\delta_{i}}\right) T^{2}\|z[k]\| .
\end{aligned}
$$

Hence, there exist constants $\gamma_{5}$ and $\gamma_{6}$ such that for small $h$ we have

$$
\left\|e_{2}[k]\right\| \leq\left(\gamma_{5}\|x[k]\|+\gamma_{6}\|z[k]\|\right) T^{2} .
$$

Now we would like to discretize equation (3.26). To this end, we note that

$$
x(t)=x(k T)+\underbrace{\int_{k T}^{t} A(\alpha(\tau)) x(\tau) d \tau+\int_{k T}^{t} B(\alpha(\tau)) u(\tau) d \tau}_{e_{3}(t)}, \quad t \in[k T,(k+1) T) .
$$


We would like to derive a bound on $\left\|e_{3}(t)\right\|$. Hence, we introduce $u_{m}[k]$ to represent the maximum magnitude of the control signal in the period $[k T,(k+1) T)$ :

$$
u_{m}[k]:=\max \left\{\left|u_{p}[k]\right|,\left|u_{s}[k]\right|\right\} .
$$

Both $u_{p}[k]$ and $u_{s}[k]$ are bounded by construction: for small $h$ we have

$$
\begin{aligned}
\left|u_{p}[k]\right| & =\rho(\|\hat{x}[k]\|+\|z[k]\|) \\
& \leq \rho\left(1+\gamma_{x} h\right)\|x[k]\|+\rho\|z[k]\|
\end{aligned}
$$

and

$$
\begin{aligned}
\left|u_{s}[k]\right| & \leq \frac{T}{T_{3}}[\|H(\hat{\alpha}[k])\|\|z[k]\|+\|K(\hat{\alpha}[k])\|\|\hat{x}[k]\|]+\frac{T_{2}}{T_{3}}\left|u_{p}[k]\right| \\
& \leq \frac{T}{T_{3}}\left[c_{h}\|z[k]\|+c_{k}\left(1+\gamma_{x} h\right)\|x[k]\|\right]+\frac{T_{2}}{T_{3}} \rho\left[\left(1+\gamma_{x} h\right)\|x[k]\|+\|z[k]\|\right] \\
& \leq\left[\frac{T}{T_{3}} c_{k}+\frac{T_{2}}{T_{3}} \rho\right]\left(1+\gamma_{x} h\right)\|x[k]\|+\left[\frac{T}{T_{3}} c_{h}+\frac{T_{2}}{T_{3}} \rho\right]\|z[k]\| .
\end{aligned}
$$

It is now easy to see that for small $h$ there exist constants $\gamma_{7}$ and $\gamma_{8}$ such that

$$
u_{m}[k] \leq \gamma_{7}\|x[k]\|+\gamma_{8}\|z[k]\| .
$$

We will also require a bound on $\|x(t)\|$ - by applying Lemma 1 for $t \in[k T,(k+1) T)$ we obtain

$$
\begin{aligned}
\|x(t)\| & \leq\left[\|x(k T)\|+(t-k T) u_{m}[k] c_{b}\right] e^{c_{a}(t-k T)} \\
& \leq\left[\|x[k]\|+T\left(\gamma_{7}\|x[k]\|+\gamma_{8}\|z[k]\|\right) c_{b}\right] e^{c_{a} T} .
\end{aligned}
$$

Hence, there exist constants $\gamma_{9}$ and $\gamma_{10}$ such that for small $h$

$$
\|x(t)\| \leq \gamma_{9}\|x[k]\|+T \gamma_{10}\|z[k]\|, \quad t \in[k T,(k+1) T) .
$$

Now we can derive a bound on $\left\|e_{3}(t)\right\|$ - for small $h$ :

$$
\begin{aligned}
\left\|e_{3}(t)\right\| & \leq \int_{k T}^{t}\|A(\alpha(\tau))\|\|x(\tau)\| d \tau+\int_{k T}^{t}\|B(\alpha(\tau))\||u(\tau)| d \tau \\
& \leq c_{a}\left(\gamma_{9}\|x[k]\|+T \gamma_{10}\|z[k]\|\right)(t-k T)+c_{b} u_{m}(t-k T) \\
& \leq\left[\left(c_{a} \gamma_{9}+c_{b} \gamma_{7}\right)\|x[k]\|+\left(T c_{a} \gamma_{10}+c_{b} \gamma_{8}\right)\|z[k]\|\right](t-k T) .
\end{aligned}
$$


Hence, there exist constants $\gamma_{11}$ and $\gamma_{12}$ such that for small $h$

$$
\left\|e_{3}(t)\right\| \leq\left(\gamma_{11}\|x[k]\|+\gamma_{12}\|z[k]\|\right)(t-k T), \quad t \in[k T,(k+1) T) .
$$

We are now set to discretize the plant state equation with the control signal applied:

$$
\begin{aligned}
x[k+1]= & x[k]+\int_{k T}^{(k+1) T} A(\alpha(\tau)) x(\tau) d \tau+\int_{k T}^{(k+1) T} B(\alpha(\tau)) u(\tau) d \tau \\
= & \left.x[k]+\int_{k T}^{(k+1) T}\left(A(\alpha(k T))+\int_{k T}^{\tau} \dot{A}(\alpha(\theta)) d \theta\right)\right) x[k] d \tau+\int_{k T}^{(k+1) T} A(\alpha(\tau)) e_{3}(\tau) d \tau \\
& \left.+\int_{k T+T_{1}}^{k T+T_{1}+T_{2}}\left(B(\alpha(k T))+\int_{k T}^{\tau} \dot{B}(\alpha(\theta)) d \theta\right)\right) u_{p}[k] d \tau \\
& \left.+\int_{k T+T_{1}+T_{2}}^{k T+T_{1}+T_{2}+T_{3}}\left(B(\alpha(k T))+\int_{k T}^{\tau} \dot{B}(\alpha(\theta)) d \theta\right)\right) u_{s}[k] d \tau \\
= & x[k]+T A(\alpha[k]) x[k]+T_{2} B(\alpha[k]) u_{p}[k]+T_{3} B(\alpha[k]) u_{s}[k] \\
& +\int_{k T}^{(k+1) T} \int_{k T}^{\tau} \dot{A}(\alpha(\theta)) d \theta x[k] d \tau+\int_{k T}^{(k+1) T} A(\alpha(\tau)) e_{3}(\tau) d \tau \\
& +\int_{k T+T_{1}}^{k T+T_{1}+T_{2}} \int_{k T}^{\tau} \dot{B}(\alpha(\theta)) d \theta u_{p}[k] d \tau+\int_{k T+T_{1}+T_{2}}^{k T+T_{1}+T_{2}+T_{3}} \int_{k T}^{\tau} \dot{B}(\alpha(\theta)) d \theta u_{s}[k] d \tau .
\end{aligned}
$$

Let us define

$$
\begin{aligned}
e_{4}[k]:= & \int_{k T}^{(k+1) T} \int_{k T}^{\tau} \dot{A}(\alpha(\theta)) d \theta x[k] d \tau+\int_{k T}^{(k+1) T} A(\alpha(\tau)) e_{3}(\tau) d \tau \\
& +\int_{k T+T_{1}}^{k T+T_{1}+T_{2}} \int_{k T}^{\tau} \dot{B}(\alpha(\theta)) d \theta u_{p}[k] d \tau+\int_{k T+T_{1}+T_{2}}^{k T+T_{1}+T_{2}+T_{3}} \int_{k T}^{\tau} \dot{B}(\alpha(\theta)) d \theta u_{s}[k] d \tau ;
\end{aligned}
$$

then using (4.52) we can write

$$
\begin{aligned}
x[k+1]= & {[I+T A(\alpha[k])] x[k]+T_{2} B(\alpha[k]) u_{p}[k] } \\
& +T_{3} B(\alpha[k])\left(\frac{T}{T_{3}}[H(\alpha[k]) z[k]+K(\alpha[k]) x[k]]-\frac{T_{2}}{T_{3}} u_{p}[k]+\frac{1}{T_{3}} e_{2}[k]\right)+e_{4}[k] \\
= & x[k]+T[A(\alpha[k])+B(\alpha[k]) K(\alpha[k])] x[k]+T B(\alpha[k]) H(\alpha[k]) z[k] \\
& +\underbrace{B(\alpha[k]) e_{2}[k]+e_{4}[k]}_{=: e_{5}[k]} .
\end{aligned}
$$


We will now derive a bound for $\left\|e_{4}[k]\right\|$ when $h$ is small:

$$
\begin{aligned}
\left\|e_{4}[k]\right\| \leq & \int_{k T}^{(k+1) T} \int_{k T}^{\tau}\|\dot{A}(\alpha(\theta))\| d \theta\|x[k]\| d \tau+\int_{k T}^{(k+1) T}\|A(\alpha(\tau))\|\left\|e_{3}(\tau)\right\| d \tau \\
& +\int_{k T+T_{1}}^{k T+T_{1}+T_{2}} \int_{k T}^{\tau}\|\dot{B}(\alpha(\theta))\| d \theta\left|u_{p}[k]\right| d \tau+\int_{k T+T_{1}+T_{2}}^{k T+T_{1}+T_{2}+T_{3}} \int_{k T}^{\tau}\|\dot{B}(\alpha(\theta))\| d \theta\left|u_{s}[k]\right| d \tau
\end{aligned}
$$

Using (4.58) to bound $\left\|e_{3}(\tau)\right\|$ and (4.56) to bound $\left|u_{p}[k]\right|$ and $\left|u_{s}[k]\right|$ we have

$$
\begin{aligned}
\left\|e_{4}[k]\right\| \leq & \int_{k T}^{(k+1) T} \delta_{A} \delta_{\alpha}\|x[k]\|(\tau-k T) d \tau+c_{a} \int_{k T}^{(k+1) T}\left[\gamma_{11}\|x[k]\|+\gamma_{12}\|z[k]\|\right](\tau-k T) d \tau \\
& +\int_{k T+T_{1}}^{k T+T_{1}+T_{2}+T_{3}} \delta_{B} \delta_{\alpha}\left[\gamma_{7}\|x[k]\|+\gamma_{8}\|z[k]\|\right](\tau-k T) d \tau \\
\leq & \delta_{A} \delta_{\alpha}\|x[k]\| \frac{T^{2}}{2}+c_{a}\left[\gamma_{11}\|x[k]\|+\gamma_{12}\|z[k]\|\right] \frac{T^{2}}{2} \\
& +\delta_{B} \delta_{\alpha}\left[\gamma_{7}\|x[k]\|+\gamma_{8}\|z[k]\|\right] \frac{T^{2}}{2}\left(1-\frac{T_{1}^{2}}{T^{2}}\right) .
\end{aligned}
$$

It is easy to see that there exist constants $\gamma_{13}$ and $\gamma_{14}$ such that for small $h$, and therefore small $T$, we have

$$
\left\|e_{4}[k]\right\| \leq\left(\gamma_{13}\|x[k]\|+\gamma_{14}\|z[k]\|\right) T^{2} .
$$

Consequently, using (4.53) and (4.60) we can derive an upper bound on $e_{5}[k]$ :

$$
\begin{aligned}
\left\|e_{5}[k]\right\| & \leq\|B(\alpha[k])\|\left\|e_{2}[k]\right\|+\left\|e_{4}[k]\right\| \\
& \leq c_{b}\left(\gamma_{5}\|x[k]\|+\gamma_{6}\|z[k]\|\right) T^{2}+\left(\gamma_{13}\|x[k]\|+\gamma_{14}\|z[k]\|\right) T^{2} \\
& =\left[\left(c_{b} \gamma_{5}+\gamma_{13}\right)\|x[k]\|+\left(c_{b} \gamma_{6}+\gamma_{14}\right)\|z[k]\|\right] T^{2} .
\end{aligned}
$$

By combining (4.50) and (4.59) we obtain the equation for the discretized closed loop system:

$$
\left[\begin{array}{c}
x[k+1] \\
z[k+1]
\end{array}\right]=\left[\begin{array}{c}
x[k] \\
z[k]
\end{array}\right]+T\left[\begin{array}{cc}
A(\alpha[k])+B(\alpha[k]) K(\alpha[k]) & B(\alpha[k]) H(\alpha[k]) \\
G(\alpha[k]) & F(\alpha[k])
\end{array}\right]\left[\begin{array}{l}
x[k] \\
z[k]
\end{array}\right]+\left[\begin{array}{l}
e_{5}[k] \\
e_{1}[k]
\end{array}\right] .
$$

Let us introduce new variables for the closed-loop state and the discretization error respectively:

$$
\xi[k]:=\left[\begin{array}{c}
x[k] \\
z[k]
\end{array}\right] \text { and } e_{6}[k]:=\left[\begin{array}{c}
e_{5}[k] \\
e_{1}[k]
\end{array}\right]
$$


The closed loop system now takes the following form:

$$
\xi[k+1]=\left(I+T\left[\begin{array}{cc}
A(\alpha[k])+B(\alpha[k]) K(\alpha[k]) & B(\alpha[k]) H(\alpha[k]) \\
G(\alpha[k]) & F(\alpha[k])
\end{array}\right]\right) \xi[k]+e_{6}[k] .
$$

We will begin by deriving an upper bound on $\left\|e_{6}[k]\right\|$. With results from (4.51) and (4.61), we can write

$$
\begin{aligned}
\left\|e_{6}[k]\right\| & =\left\|e_{1}[k]\right\|+\left\|e_{5}[k]\right\| \\
& \leq\left[\gamma_{3}\|x[k]\|+\gamma_{4}\|z[k]\|+\left(c_{b} \gamma_{5}+\gamma_{13}\right)\|x[k]\|+\left(c_{b} \gamma_{6}+\gamma_{14}\right)\|z[k]\|\right] T^{2} .
\end{aligned}
$$

Noting that $\|\xi[k]\|=\|x[k]\|+\|z[k]\|$, it follows that there exists a constant $\gamma_{15}$ such that for small $T$

$$
\left\|e_{6}[k]\right\| \leq \gamma_{15} T^{2}\|\xi[k]\|
$$

At this point we are ready to prove the stability of the closed loop system. An important result is induced from equation (3.25):

$$
\left[\begin{array}{cc}
A(\alpha[k])+B(\alpha[k]) K(\alpha[k]) & B(\alpha[k]) H(\alpha[k]) \\
G(\alpha[k]) & F(\alpha[k])
\end{array}\right]=\left[\begin{array}{c}
X \\
Z
\end{array}\right] P(\alpha[k])\left[\begin{array}{c}
X \\
Z
\end{array}\right]^{-1} .
$$

Substituting the above into (4.63) yields

$$
\xi[k+1]=\left(I+T\left[\begin{array}{c}
X \\
Z
\end{array}\right] P(\alpha[k])\left[\begin{array}{c}
X \\
Z
\end{array}\right]^{-1}\right) \xi[k]+e_{6}[k] .
$$

Defining the following transformations:

$$
\bar{\xi}[k]:=\left[\begin{array}{c}
X \\
Z
\end{array}\right]^{-1} \xi[k] \quad \text { and } \quad \bar{e}_{6}[k]:=\left[\begin{array}{c}
X \\
Z
\end{array}\right]^{-1} e_{6}[k],
$$

(4.66) accordingly becomes

$$
\bar{\xi}[k+1]=[I+T P(\alpha[k])] \bar{\xi}[k]+\bar{e}_{6}[k],
$$

and

$$
\begin{aligned}
\left\|\bar{e}_{6}[k]\right\| & \leq\left\|\left[\begin{array}{c}
X \\
Z
\end{array}\right]^{-1}\right\|\left\|e_{6}[k]\right\| \\
& \leq\left\|\left[\begin{array}{c}
X \\
Z
\end{array}\right]^{-1}\right\| \gamma_{15} T^{2}\|\xi[k]\| \\
& \leq \underbrace{\left\|\left[\begin{array}{c}
X \\
Z
\end{array}\right]^{-1}\right\|\left\|\left[\begin{array}{c}
X \\
Z
\end{array}\right]\right\| \gamma_{15} T^{2}\|\bar{\xi}[k]\| .}_{=: \gamma_{16}}
\end{aligned}
$$


By Proposition 1 there exist $\bar{T}>0$ and $\bar{\lambda}>0$ so that for all $T \in(0, \bar{T})$ and $\lambda \in(0, \bar{\lambda})$ we have

$$
\|I+T P(\alpha[k])\| \leq 1-\lambda T \text { for all } \alpha[k] \in \mathcal{A} .
$$

Hence fix $\lambda \in(0, \bar{\lambda})$ and let $T \in(0, \bar{T})$ be arbitrary; taking the norm of the both sides of (4.68) we have

$$
\begin{aligned}
\|\bar{\xi}[k+1]\| & \leq\|I+T P(\alpha[k])\|\|\bar{\xi}[k]\|+\left\|\bar{e}_{6}[k]\right\| \\
& \leq(1-\lambda T)\|\bar{\xi}[k]\|+\gamma_{15} \gamma_{16} T^{2}\|\bar{\xi}[k]\| .
\end{aligned}
$$

We would like to show that $\left\|\bar{e}_{6}[k]\right\|$ is small enough to not affect the closed loop stability for a sufficiently small $T$. We would like to find a $T^{*} \in(0, \bar{T})$ such that for all $T \in\left(0, T^{*}\right)$ we have

$$
\gamma_{15} \gamma_{16} T^{2} \leq \frac{\lambda}{2} T
$$

Obviously, we can choose

$$
T^{*}=\min \left\{\frac{\lambda}{2 \gamma_{15} \gamma_{16}}, \frac{\bar{T}}{2}\right\} .
$$

Hence, for small $T$, if we use (4.70) in (4.69) we derive the following bound on $\|\bar{\xi}[k+1]\|$ :

$$
\begin{aligned}
\|\bar{\xi}[k+1]\| & \leq(1-\lambda T)\|\bar{\xi}[k]\|+\frac{\lambda}{2} T \bar{\xi}[k] \| \\
& \leq\left(1-\frac{\lambda}{2} T\right)\|\bar{\xi}[k]\| \\
& \leq e^{-\frac{\lambda}{2} T}\|\bar{\xi}[k]\| .
\end{aligned}
$$

Recall that at the beginning of the section we made the assumption that we are considering only those $k$ for which $\left\|\left[\begin{array}{l}x[k] \\ z[k]\end{array}\right]\right\|>0$. If $\left\|\left[\begin{array}{l}x[k] \\ z[k]\end{array}\right]\right\|=0$, then $\hat{x}[k]=0$, so $u_{p}[k]=u_{s}[k]=0$, so $\left\|\left[\begin{array}{l}x[k+1] \\ z[k+1]\end{array}\right]\right\|=0$, i.e. $\|\bar{\xi}[k+1]\|=0$. Therefore, it is true that

$$
\|\bar{\xi}[k+1]\| \leq e^{-\frac{\lambda}{2} T}\|\bar{\xi}[k]\|, \quad k \in \mathbb{Z}^{+} .
$$

Consequently,

$$
\|\bar{\xi}[k]\| \leq e^{-\frac{\lambda}{2} k T}\|\bar{\xi}[0]\|, \quad k \in \mathbb{Z}^{+} .
$$


We would like to return to the original domain and derive a similar bound on $\|\xi[k]\|$ :

$$
\begin{aligned}
\|\xi[k]\| & =\left\|\left[\begin{array}{l}
X \\
Z
\end{array}\right] \bar{\xi}[k]\right\| \\
& \leq\left\|\left[\begin{array}{c}
X \\
Z
\end{array}\right]\right\|\|\bar{\xi}[k]\| \\
& \leq\left\|\left[\begin{array}{c}
X \\
Z
\end{array}\right]\right\| e^{-\frac{\lambda}{2} k T}\|\bar{\xi}[0]\| \\
& \leq \gamma_{16} e^{-\frac{\lambda}{2} k T}\|\xi[0]\|, \quad k \in \mathbb{Z}^{+} .
\end{aligned}
$$

This means that the state of the closed loop system is bounded by a decaying exponential at the sample points. The only thing left to show is that the state remains bounded in between the samples.

\subsection{Intersample Behaviour}

Now we will investigate the behaviour of the closed loop state in between the sample points. We will define the following continuous time signals:

$$
\begin{gathered}
z(t):=z[k] \quad \text { for } \quad t \in[k T,(k+1) T), \\
\xi(t):=\left[\begin{array}{l}
x(t) \\
z(t)
\end{array}\right] .
\end{gathered}
$$

Our goal is to derive an upper bound on $\|\xi(t)\|$ when $t \in[k T,(k+1) T)$. An upper bound for $\|x(t)\|$ when $t \in[k T,(k+1) T)$ was derived in (4.57). Noting that $z(t)$ remains constant for the duration of the period, we have the following result:

$$
\begin{aligned}
\left\|\left[\begin{array}{l}
x(t) \\
z(t)
\end{array}\right]\right\| & =\|x(t)\|+\|z(t)\| \\
& \leq \gamma_{9}\|x[k]\|+\gamma_{10} T\|z[k]\|+\|z[k]\| \\
& \leq \underbrace{\left(\gamma_{9}+\gamma_{10} T^{*}+1\right)}_{=: \gamma_{17}}\left\|\left[\begin{array}{l}
x[k] \\
z[k]
\end{array}\right]\right\|, \quad t \in[k T,(k+1) T) .
\end{aligned}
$$

Combining the above with (4.73) yields the bound on $\|\xi(t)\|$ when $t \in[k T,(k+1) T)$ and when $T$ is small:

$$
\begin{aligned}
\|\xi(t)\| & \leq \gamma_{17}\|\xi[k]\| \\
& \leq \gamma_{16} \gamma_{17} e^{-\frac{\lambda}{2} k T}\|\xi[0]\| \\
& =\gamma_{16} \gamma_{17} e^{-\frac{\lambda}{2} k T}\|\xi(0)\| .
\end{aligned}
$$


This result can be easily extended to show that $\|\xi(t)\|$ is bounded for all $t \in \mathbb{R}^{+}$. First, note that when $t \in[k T,(k+1) T]$, we have $e^{-\frac{\lambda}{2}(t-T)} \in\left[e^{-\frac{\lambda}{2} k T}, e^{-\frac{\lambda}{2}(k-1) T}\right]$. Using this observation in (4.74), we obtain the desired result:

$$
\begin{aligned}
\|\xi(t)\| & \leq \gamma_{16} \gamma_{17} e^{-\frac{\lambda}{2}(t-T)}\|\xi(0)\| \\
& \leq \gamma_{16} \gamma_{17} e^{\frac{\lambda}{2} T} e^{-\frac{\lambda}{2} t}\|\xi(0)\| \\
& \leq \underbrace{\gamma_{16} \gamma_{17} e^{\frac{\lambda}{2}} T^{*}}_{=: \gamma_{18}} e^{-\frac{\lambda}{2} t}\|\xi(0)\|, \quad t \in \mathbb{R}^{+} .
\end{aligned}
$$

\subsection{Summary}

In this chapter we considered the performance of the closed loop system in the absence of noise. We began by examining the bound on the state estimation error, which turned out to be

$$
\|\hat{x}[k]-x[k]\| \leq \gamma_{x}\|x[k]\| h .
$$

This means that the difference between the plant's state and its estimate can be made small by means of the fast sampling rate. Using this result, we showed that if $\left\|\left[\begin{array}{l}x[k] \\ z[k]\end{array}\right]\right\|>0$ and assuming no jumps in the parameter, the bound on the parameter estimation error is

$$
|\hat{\alpha}[k]-\alpha[k]| \leq\left(n \delta_{\alpha}+\frac{\gamma_{f}}{\delta_{i}}\right) h .
$$

The above bounds are fairly conservative; however, we were able to show that when these estimated quantities are used to calculate the control signal, the resulting closed loop system is exponentially stable, i.e.

$$
\|\xi(t)\| \leq \gamma_{18} e^{-\frac{\lambda}{2} t}\|\xi(0)\|, \quad t \in \mathbb{R}^{+} .
$$

In the derivation of the above bound we assumed that there were no discontinuities in $\alpha(t)$. Having ascertained that the behaviour of the closed loop system is nice in the absence of noise and parameter jumps, we will move on to analysing the effects of these disturbances in the next chapter. 


\section{Chapter 5}

\section{System in Presence of Noise}

We are now ready to consider the situation where noise is injected into the system at the input and output interfaces as shown in Figure 5.1. The goal of the current chapter is to show that the estimation techniques proposed in the previous chapter remain valid and that the closed loop system is stable according to our definition. Many of the results from the noise free case will be very useful in the present setup due to the linear nature of the controlled system.

Incorporating the input and output noise signals into the plant model yields

$$
\begin{aligned}
\dot{x}(t) & =A(\alpha(t)) x(t)+B(\alpha(t)) \underbrace{[u(t)+d(t)]}_{u_{d}(t)}, \quad x(0)=x_{0} \\
y_{w}(t) & =C x(t)+w(t) .
\end{aligned}
$$

We will first consider the homogeneous version of our system:

$$
\dot{x}(t)=A(\alpha(t)) x(t), \quad x\left(t_{0}\right)=x_{0} .
$$

By uniqueness and existence of the solution, for $t_{0}>0$ there exists a state transition matrix, $\Phi_{A}\left(t, t_{0}\right)$, such that, for a given initial condition $x\left(t_{0}\right)$, the solution to (5.3) is

$$
x(t)=\Phi_{A}\left(t, t_{0}\right) x\left(t_{0}\right), \quad t \geq t_{0} .
$$

We will derive a useful intermediate result by applying Lemma 1 to the above equation:

$$
\begin{aligned}
&\left\|\Phi_{A}\left(t, t_{0}\right) x\left(t_{0}\right)\right\| \leq\left\|x\left(t_{0}\right)\right\| e^{c_{a}\left(t-t_{0}\right)} \\
& \Longrightarrow \frac{\left\|\Phi_{A}\left(t, t_{0}\right) x\left(t_{0}\right)\right\|}{\left\|x\left(t_{0}\right)\right\|} \leq e^{c_{a}\left(t-t_{0}\right)}, \quad t \geq t_{0} .
\end{aligned}
$$




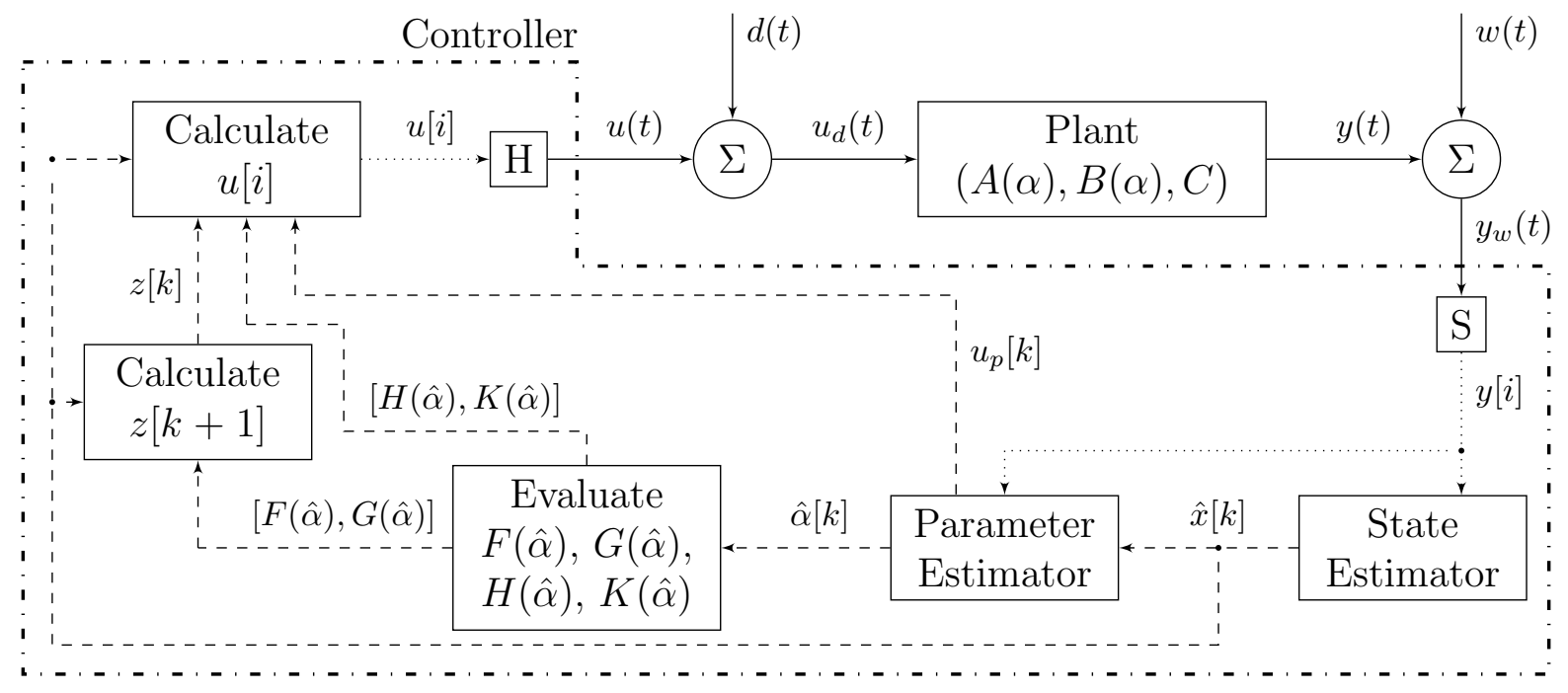

Figure 5.1: System setup with noise injected at the input and output interfaces.

Since (5.5) holds true for all $x\left(t_{0}\right) \neq 0$, we say

$$
\sup _{x\left(t_{0}\right) \neq 0} \frac{\left\|\Phi_{A}\left(t, t_{0}\right) x\left(t_{0}\right)\right\|}{\left\|x\left(t_{0}\right)\right\|} \leq e^{c_{a}\left(t-t_{0}\right)}, \quad t \geq t_{0}
$$

or

$$
\left\|\Phi_{A}\left(t, t_{0}\right)\right\| \leq e^{c_{a}\left(t-t_{0}\right)}, \quad t \geq t_{0} .
$$

In the nonhomogeneous case, the expressions for the state and output are, respectively,

$$
\begin{aligned}
x(t) & =\Phi_{A}\left(t, t_{0}\right) x\left(t_{0}\right)+\int_{t_{0}}^{t} \Phi_{A}(t, \tau) B(\tau)[u(\tau)+d(\tau)] d \tau, \\
y_{w}(t) & =C \Phi_{A}\left(t, t_{0}\right) x\left(t_{0}\right)+C \int_{t_{0}}^{t} \Phi_{A}(t, \tau) B(\tau)[u(\tau)+d(\tau)] d \tau+w(t) .
\end{aligned}
$$

Before proceeding, we need to make an additional assumption about the noise signals. Since our goal is to prove that the map from $\left[\begin{array}{l}d \\ w\end{array}\right]$ to $\left[\begin{array}{l}y \\ u\end{array}\right]$ (with zero initial conditions) is bounded, in this chapter we assume that the noise signals, $d(t)$ and $w(t)$, are bounded:

$$
\|d\|_{\infty}<\infty \text { and }\|w\|_{\infty}<\infty .
$$

We will define a new noise vector:

$$
\bar{w}(t)=\left[\begin{array}{l}
d(t) \\
w(t)
\end{array}\right]
$$


so it follows immediately that

$$
\|d\|_{\infty} \leq\|\bar{w}\|_{\infty} \quad \text { and } \quad\|w\|_{\infty} \leq\|\bar{w}\|_{\infty}
$$

\section{$5.1 \quad$ State Estimation}

We will estimate the state by setting $u(t)=0$ for $t \in[k T, k T+n h)$, the same as in the noise free scenario. Notice that the noise enters linearly into the system and so it is possible to consider its effect separately. On that basis, we will define $y_{i c}(t)$ to be the response of the system to the initial condition (i.e. setting $d(t)=0$ and $w(t)=0$ ):

$$
y_{i c}(t):=C \Phi_{A}(t, k T) x(k T) .
$$

Similarly, the response due to the noise when $x(k T)=0$ is

$$
y_{N}(t):=C \int_{k T}^{t} \Phi_{A}(t, \tau) B(\tau) d(\tau) d \tau+w(t)
$$

Due to linearity of equations (5.7)-(5.8), we can write the total response as the addition of $y_{i c}(t)$ and $y_{N}(t)$ :

$$
y_{w}(t)=y_{i c}(t)+y_{N}(t)
$$

Let us define

$$
\mathcal{Y}_{k}^{w}:=\left[\begin{array}{c}
y_{w}(k T) \\
y_{w}(k T+h) \\
\vdots \\
y_{w}(k T+(n-1) h)
\end{array}\right]
$$

and

$$
\mathcal{Y}_{k}^{i c}:=\left[\begin{array}{c}
y_{i c}(k T) \\
y_{i c}(k T+h) \\
\vdots \\
y_{i c}(k T+(n-1) h)
\end{array}\right], \quad \mathcal{Y}_{k}^{N}:=\left[\begin{array}{c}
y_{N}(k T) \\
y_{N}(k T+h) \\
\vdots \\
y_{N}(k T+(n-1) h)
\end{array}\right]
$$

Note that the response to the initial condition is identical to the output with $u(t)=0$ in the noise free case (Section 4.1). Therefore, using equation (4.17) (and the definition of $\mathcal{V}_{k}$ given in (4.14)) we can write

$$
\mathcal{Y}_{k}^{i c}=S_{n} Q_{n}(h) \mathcal{O}(C, A(\alpha)) x[k]+\mathcal{V}_{k}
$$


The input to the sampler in Figure 5.1 is $y_{w}(t)$ and so we can write the estimation law in a slightly different way than in (4.19):

$$
\begin{aligned}
\hat{x}[k] & =\mathcal{O}^{-1}(C, A(\alpha)) Q_{n}^{-1}(h) S_{n}^{-1} \mathcal{Y}_{k}^{w} \\
& =\mathcal{O}^{-1}(C, A(\alpha)) Q_{n}^{-1} S_{n}^{-1}\left(\mathcal{Y}_{k}^{i c}+\mathcal{Y}_{k}^{N}\right) .
\end{aligned}
$$

Hence, using (5.14) the state estimation error is

$$
\begin{aligned}
\|\hat{x}[k]-x[k]\| & =\left\|\mathcal{O}^{-1}(C, A(\alpha)) Q_{n}^{-1} S_{n}^{-1}\left[\mathcal{Y}_{k}^{N}+\mathcal{V}_{k}\right]\right\| \\
& \leq\left\|\mathcal{O}^{-1}(C, A(\alpha))\right\|\left\|Q_{n}^{-1}\right\|\left\|S_{n}^{-1}\right\|\left[\left\|\mathcal{Y}_{k}^{N}\right\|+\left\|\mathcal{V}_{k}\right\|\right] .
\end{aligned}
$$

Now we would like to derive an upper bound on $\left\|\mathcal{Y}_{k}^{N}\right\|$. We will first consider $\left|y_{N}(t)\right|$ when $t \in[k T, k T+n h):$

$$
\begin{aligned}
\left|y_{N}(t)\right| & =\left|C \int_{k T}^{t} \Phi_{A}(t, \tau) B(\tau) d(\tau) d \tau+w(t)\right| \\
& \leq\|C\| \int_{k T}^{t}\left\|\Phi_{A}(t, \tau)\right\|\|B(\tau)\||d(\tau)| d \tau+|w(t)| \\
& \leq\|C\| c_{b}\|d\|_{\infty} \int_{k T}^{t} e^{c_{a}(t-\tau)} d \tau+\|w\|_{\infty} \\
& \leq\|C\| c_{b} e^{c_{a} n h}\|d\|_{\infty}(t-k T)+\|w\|_{\infty} \\
& \leq\left[\|C\| c_{b} e^{c_{a} n h}(t-k T)+1\right]\|\bar{w}\|_{\infty}, \quad t \in[k T, k T+n h) .
\end{aligned}
$$

Now we can bound individual elements of $\mathcal{Y}_{k}^{N}$ :

$$
\begin{aligned}
\left|y_{N}(k T)\right| & =0 \\
\left|y_{N}(k T+h)\right| & \leq\left[\|C\| c_{b} e^{c_{a} n h} h+1\right]\|\bar{w}\|_{\infty}, \\
\left|y_{N}(k T+2 h)\right| & \leq\left[\|C\| c_{b} e^{c_{n} n h} 2 h+1\right]\|\bar{w}\|_{\infty}, \\
& \vdots \\
\left|y_{N}(k T+(n-1) h)\right| & \leq\left[\|C\| c_{b} e^{c_{n} n h}(n-1) h+1\right]\|\bar{w}\|_{\infty} .
\end{aligned}
$$

From here it is easy to derive the upper bound on $\left\|\mathcal{Y}_{k}^{N}\right\|$ :

$$
\left\|\mathcal{Y}_{k}^{N}\right\| \leq\left[\|C\| c_{b} e^{c_{a} n h} \frac{n}{2} h+1\right](n-1)\|\bar{w}\|_{\infty} .
$$


Clearly, there exists a constant $\gamma_{19}$ such that for small $h$

$$
\left\|\mathcal{Y}_{k}^{N}\right\| \leq \gamma_{19}\|\bar{w}\|_{\infty} .
$$

Recall that from the noise free case (equations (4.20)-(4.24)) we have

$$
\left\|\mathcal{O}^{-1}(C, A(\alpha))\right\|\left\|Q_{n}^{-1}(h)\right\|\left\|S_{n}^{-1}\right\|\left\|\mathcal{V}_{k}\right\| \leq \gamma_{x}\|x[k]\| h .
$$

Hence, substituting (5.17) and (4.21) into (5.16), we obtain for small $h$

$$
\|\hat{x}[k]-x[k]\| \leq \gamma_{x}\|x[k]\| h+\left\|\mathcal{O}^{-1}(C, A(\alpha))\right\|\left\|S_{n}^{-1}\right\| \frac{(n-1) !}{h^{n-1}} \gamma_{19}\|\bar{w}\|_{\infty} .
$$

Letting

$$
\gamma_{20}:=\left\|\mathcal{O}^{-1}(C, A(\alpha))\right\|\left\|S_{n}^{-1}\right\|(n-1) ! \gamma_{19},
$$

we arrive at the upper bound on the state estimation error:

$$
\|\hat{x}[k]-x[k]\| \leq \gamma_{x}\|x[k]\| h+\frac{\gamma_{20}}{h^{n-1}}\|\bar{w}\|_{\infty} .
$$

\subsection{Parameter Estimation}

The parameter estimation procedure will not change from the noise free case; once again we proceed on the assumption that $\alpha \in A C(\mathcal{A})$. A probing control signal $u_{p}[k]=$ $\rho(\|\hat{x}[k]\|+\|z[k]\|)$ will be applied for $t \in\left[\bar{t}_{1}, \bar{t}_{1}+(\bar{n}+2) h\right)$. As it was pointed out in the previous section, taking advantage of the linearity of the plant simplifies the analysis. Here we will isolate the effect of noise in the similar manner. Let $y_{i p}(t)$ be the response when $d(t)=0$ and $w(t)=0$ (i.e. it is a response to the initial condition and probing input):

$$
y_{i p}(t)=C \Phi_{A}\left(t, \bar{t}_{1}\right) x\left(\bar{t}_{1}\right)+C \int_{\bar{t}_{1}}^{t} \Phi_{A}(t, \tau) B(\tau) u_{p}[k] d \tau .
$$

Similarly, the response due to noise inputs is

$$
\bar{y}_{N}(t)=C \int_{\bar{t}_{1}}^{t} \Phi_{A}(t, \tau) B(\tau) d(\tau) d \tau+w(t) .
$$

Once again, it is easy to see that for $t \in\left[\bar{t}_{1}, \bar{t}_{1}+(\bar{n}+2) h\right)$,

$$
y_{w}(t)=y_{i p}(t)+\bar{y}_{N}(t) .
$$


We will introduce the following vectors of samples:

$$
\overline{\mathcal{Y}}_{k}^{w}:=\left[\begin{array}{c}
y_{w}\left(\bar{t}_{1}\right) \\
y_{w}\left(\bar{t}_{1}+h\right) \\
\vdots \\
y_{w}\left(\bar{t}_{1}+(\bar{n}+1) h\right)
\end{array}\right]
$$

and

$$
\mathcal{Y}_{k}^{i p}:=\left[\begin{array}{c}
y_{i p}\left(\bar{t}_{1}\right) \\
y_{i p}\left(\bar{t}_{1}+h\right) \\
\vdots \\
y_{i p}\left(\bar{t}_{1}+(\bar{n}+1) h\right)
\end{array}\right], \quad \overline{\mathcal{Y}}_{k}^{N}:=\left[\begin{array}{c}
\bar{y}_{N}\left(\bar{t}_{1}\right) \\
\bar{y}_{N}\left(\bar{t}_{1}+h\right) \\
\vdots \\
\bar{y}_{N}\left(\bar{t}_{1}+(\bar{n}+1) h\right)
\end{array}\right] .
$$

Observing that $y_{i p}(t)$ is equal to the output in the noise free case when $u(t)=u_{p}[k]$ (Section 4.2) and using (4.33) (with $\mathcal{M}_{k}$ defined in (4.31)), we can write

$$
f\left(\alpha\left(\bar{t}_{1}\right)\right)=\frac{1}{u_{p}[k]}\left[E Q_{\bar{n}+2}^{-1}(h) S_{\bar{n}+2}^{-1}\left(\mathcal{Y}_{k}^{i p}-\mathcal{M}_{k}\right)-C A^{\bar{n}+1} x\left(\bar{t}_{1}\right)\right] .
$$

We will define $\check{f}[k]$ similarly to equation (4.34), with a slight modification due to noise:

$$
\check{f}[k]:=\left\{\begin{array}{cl}
\frac{1}{u_{p}[k]}\left[E Q_{\bar{n}+2}^{-1}(h) S_{\bar{n}+2}^{-1} \overline{\mathcal{Y}}_{k}^{w}-C A^{\bar{n}+1} \hat{x}[k]\right] & \text { if } u_{p}[k] \neq 0 ; \\
f(\underline{\alpha}) & \text { if } u_{p}[k]=0 .
\end{array}\right.
$$

The definitions of $\hat{f}[k]$ and $\hat{\alpha}\left(\bar{t}_{1}\right)$ will remain the same as in (4.35) and (4.36) respectively:

$$
\hat{f}[k]:=\Pi_{\mathcal{F}}(\check{f}[k]) .
$$

and

$$
\hat{\alpha}[k]:=f^{-1}(\hat{f}[k])
$$

We now proceed to derive a bound on the estimation error. To this end, we will first consider the situation when $u_{p}[k]=0$, i.e. $\hat{x}[k]=0$ and $z[k]=0$. This means that $\hat{\alpha}[k]=\underline{\alpha}$, which, in general, is not an accurate estimate of $\alpha\left(\bar{t}_{1}\right)$. Let us examine $\|x[k]\|$ in such case:

$$
\begin{aligned}
\|x[k]\| & \leq\|x[k]-\hat{x}[k]\|+\|\hat{x}[k]\| \\
& \leq \gamma_{x}\|x[k]\| h+\frac{\gamma_{20}}{h^{n-1}}\|\bar{w}\|_{\infty}+\|\hat{x}[k]\| .
\end{aligned}
$$


If we choose $h<\frac{1}{\gamma_{x}}$, we have

$$
\|x[k]\| \leq \frac{1}{1-\gamma_{x} h}\left[\frac{\gamma_{20}}{h^{n-1}}\|\bar{w}\|_{\infty}+\|\hat{x}[k]\|\right]
$$

SO

$$
\|x[k]\| \leq \frac{\gamma_{20}}{\left(1-\gamma_{x} h\right) h^{n-1}}\|\bar{w}\|_{\infty} .
$$

Therefore, we see that if $u_{p}[k]=0$, then for small $h$ we have

$$
\left\|\left[\begin{array}{l}
x[k] \\
z[k]
\end{array}\right]\right\| \leq \frac{\gamma_{20}}{\left(1-\gamma_{x} h\right) h^{n-1}}\|\bar{w}\|_{\infty} .
$$

This suggests that, in particular, if $h<1$ is small enough, then

$$
\left\|\left[\begin{array}{l}
x[k] \\
z[k]
\end{array}\right]\right\|>\frac{\gamma_{20}}{\left(1-\gamma_{x} h\right) h^{n}}\|\bar{w}\|_{\infty}
$$

implies that $u_{p}[k] \neq 0$, in which case

$$
\begin{aligned}
\check{f}[k] & =\frac{1}{u_{p}[k]}\left[E Q_{\bar{n}+2}^{-1}(h) S_{\bar{n}+2}^{-1} \overline{\mathcal{Y}}_{k}^{w}-C A^{\bar{n}+1} \hat{x}[k]\right] \\
& =\frac{1}{u_{p}[k]}\left[E Q_{\bar{n}+2}^{-1}(h) S_{\bar{n}+2}^{-1}\left(\mathcal{Y}_{k}^{i p}+\overline{\mathcal{Y}}_{k}^{N}\right)-C A^{\bar{n}+1} \hat{x}[k]\right]
\end{aligned}
$$

Therefore, for the remainder of the section we will assume that (5.29) is true.

Letting $\tilde{f}[k]=\check{f}[k]-f\left(\alpha\left(\bar{t}_{1}\right)\right)$, using (5.24) and (5.30) we can obtain a bound on $|\tilde{f}[k]|$ :

$$
|\tilde{f}[k]| \leq \frac{1}{\left|u_{p}[k]\right|}\left[\left\|C A^{\bar{n}+1}\right\|\left\|\hat{x}[k]-x\left(\bar{t}_{1}\right)\right\|+\|E\|\left\|Q_{\bar{n}+2}^{-1}(h)\right\|\left\|S_{\bar{n}+2}^{-1}\right\|\left(\left\|\overline{\mathcal{Y}}_{k}^{N}\right\|+\left\|\mathcal{M}_{k}\right\|\right)\right] .
$$

Firstly, let us derive a bound on $\left\|\hat{x}[k]-x\left(\bar{t}_{1}\right)\right\|$ :

$$
\begin{aligned}
\left\|\hat{x}[k]-x\left(\bar{t}_{1}\right)\right\| & =\| \hat{x}[k]-\left(x(k T)+\int_{k T}^{k T+n h} A(\alpha(\tau) x(\tau) d \tau) \|\right. \\
& \leq\|\hat{x}[k]-x[k]\|+\int_{k T}^{k T+n h} \| A(\alpha(\tau)\|\| x(\tau) \| d \tau .
\end{aligned}
$$

We will use Lemma 1 to obtain a bound on $\|x(\tau)\|$ when $\tau \in[k T, k T+n h)$ :

$$
\begin{aligned}
\|x(\tau)\| & \leq\left(\|x(k T)\|+(\tau-k T) c_{b}\|d\|_{\infty}\right) e^{c_{a}(\tau-k T)} \\
& \leq\left(\|x[k]\|+n h c_{b}\|\bar{w}\|_{\infty}\right) e^{c_{a} n h} .
\end{aligned}
$$


Using (5.18), this yields

$$
\begin{aligned}
\left\|\hat{x}[k]-x\left(\bar{t}_{1}\right)\right\| & \leq \gamma_{x}\|x[k]\| h+\frac{\gamma_{20}}{h^{n-1}}\|\bar{w}\|_{\infty}+c_{a} \int_{k T}^{k T+n h}\left(\|x[k]\|+n h c_{b}\|\bar{w}\|_{\infty}\right) e^{c_{a} n h} d \tau \\
& \leq\left(\gamma_{x}+n c_{a} e^{c_{a} n h}\right)\|x[k]\| h+\left[\frac{\gamma_{20}}{h^{n-1}}+c_{a} e^{c_{a} n h} n^{2} h^{2} c_{b}\right]\|\bar{w}\|_{\infty} .
\end{aligned}
$$

It is easy to see that there exist constants $\gamma_{21}$ and $\gamma_{22}$ such that for small $h$ we have

$$
\left\|\hat{x}[k]-x\left(\bar{t}_{1}\right)\right\| \leq \gamma_{21}\|x[k]\| h+\frac{\gamma_{22}}{h^{n-1}}\|\bar{w}\|_{\infty} .
$$

We also require an upper bound on $\left\|\overline{\mathcal{Y}}_{k}^{N}\right\|$. To this end, consider $\left|\bar{y}_{N}(t)\right|$ when $t \in\left[\bar{t}_{1}, \bar{t}_{1}+\right.$ $(\bar{n}+2) h)$ :

$$
\begin{aligned}
\left|\bar{y}_{N}(t)\right| & \leq\|C\| \int_{\bar{t}_{1}}^{t}\left\|\Phi_{A}(t, \tau)\right\|\|B(\tau)\|\|d(\tau)\| d \tau+\|w(t)\| \\
& \leq\|C\| c_{b}\|d\|_{\infty} \int_{\bar{t}_{1}}^{t} e^{c_{a}(t-\tau)} d \tau+\|w\|_{\infty} \\
& \leq\|C\| c_{b} e^{c_{a}(\bar{n}+2) h}\left(t-\bar{t}_{1}\right)\|d\|_{\infty}+\|w\|_{\infty} \\
& \leq\left[\|C\| c_{b} e^{c_{a}(\bar{n}+2) h}\left(t-\bar{t}_{1}\right)+1\right]\|\bar{w}\|_{\infty} .
\end{aligned}
$$

This result can be used to bound the individual elements of $\overline{\mathcal{Y}}_{k}^{N}$ :

$$
\begin{aligned}
\left|\bar{y}_{N}\left(\bar{t}_{1}\right)\right| & =0 \\
\left|\bar{y}_{N}\left(\bar{t}_{1}+h\right)\right| & \leq\left[\|C\| c_{b} e^{c_{a}(\bar{n}+2) h} h+1\right]\|\bar{w}\|_{\infty}, \\
\left|\bar{y}_{N}\left(\bar{t}_{1}+2 h\right)\right| & \leq\left[\|C\| c_{b} e^{c_{a}(\bar{n}+2) h} 2 h+1\right]\|\bar{w}\|_{\infty}, \\
& \vdots \\
\left|\bar{y}_{N}\left(\bar{t}_{1}+(\bar{n}+1) h\right)\right| & \leq\left[\|C\| c_{b} e^{c_{a}(\bar{n}+2) h}(\bar{n}+1) h+1\right]\|\bar{w}\|_{\infty} .
\end{aligned}
$$

The bound on $\left\|\overline{\mathcal{Y}}_{k}^{N}\right\|$ is now easily obtained:

$$
\left\|\overline{\mathcal{Y}}_{k}^{N}\right\| \leq\left[\|C\| c_{b} e^{c_{a}(\bar{n}+2) h} \frac{\bar{n}+2}{2} h+1\right](\bar{n}+1)\|\bar{w}\|_{\infty} .
$$

Obviously, there exists a constant $\gamma_{23}$ such that for small $h$

$$
\left\|\overline{\mathcal{Y}}_{k}^{N}\right\| \leq \gamma_{23}\|\bar{w}\|_{\infty}
$$


Using (4.41) to produce a bound on $\left\|\mathcal{M}_{k}\right\|,(4.42)$ to produce bounds on $\|E\|$ and $\left\|Q_{\bar{n}+2}^{-1}(h)\right\|$, (5.33) and (5.34) in (5.31) yields the following for small $h$ :

$$
\begin{aligned}
|\tilde{f}[k]| \leq & \frac{1}{\left|u_{p}[k]\right|}\left\{\left\|C A^{\bar{n}+1}\right\|\left(\gamma_{21}\|x[k]\| h+\frac{\gamma_{22}}{h^{n-1}}\|\bar{w}\|_{\infty}\right)\right. \\
& \left.+\left\|S_{\bar{n}+2}^{-1}\right\| \frac{(\bar{n}+1) !}{h^{\bar{n}+1}}\left[\gamma_{\mu}\left(\gamma_{1}\|x[k]\|+\gamma_{2}\left|u_{p}[k]\right|\right) \frac{h^{\bar{n}+2}}{(\bar{n}+2) !}+\gamma_{23}\|\bar{w}\|_{\infty}\right]\right\}
\end{aligned}
$$

using the fact that $n-\bar{n}-2 \geq 0$ (see Assumption 4), there exists a constant $\gamma_{24}$ so that for small $h$ :

$$
|\tilde{f}[k]| \leq \frac{\gamma_{24}}{u_{p}[k]}\left[\|x[k]\| h+\left|u_{p}[k]\right| h+\frac{1}{h^{n-1}}\|\bar{w}\|_{\infty}\right] .
$$

Using (5.26) this becomes, for small $h$

$$
|\tilde{f}[k]| \leq \frac{\gamma_{24}}{\left|u_{p}[k]\right|}\left[\frac{h}{1-\gamma_{x} h}\left[\frac{\gamma_{20}}{h^{n-1}}\|\bar{w}\|_{\infty}+\|\hat{x}[k]\|\right]+\left|u_{p}[k]\right| h+\frac{1}{h^{n-1}}\|\bar{w}\|_{\infty}\right] .
$$

Hence, there exists a constant $\gamma_{25}$ such that for small $h$ :

$$
|\tilde{f}[k]| \leq \frac{\gamma_{25}}{\left|u_{p}[k]\right|}\left[\frac{\rho}{1+\rho}\left(\|\hat{x}[k]\|+\left|u_{p}[k]\right|\right) h+\rho(1-h) \frac{1}{h^{n-1}}\|\bar{w}\|_{\infty}\right] .
$$

We would like to relate the size of $u_{p}[k]$ to $\|\bar{w}\|_{\infty}$. To this end, from (5.26) we obtain for small $h$

$$
\|\hat{x}[k]\| \geq\left(1-\gamma_{x} h\right)\|x[k]\|-\frac{\gamma_{20}}{h^{n-1}}\|\bar{w}\|_{\infty} .
$$

Also, from (5.29) we have that

$$
\begin{aligned}
\|x[k]\|+\|z[k]\| & >\frac{\gamma_{20}}{\left(1-\gamma_{x} h\right) h^{n}}\|\bar{w}\|_{\infty} \\
\Longrightarrow\|x[k]\| & >\frac{\gamma_{20}}{\left(1-\gamma_{x} h\right) h^{n}}\|\bar{w}\|_{\infty}-\|z[k]\|,
\end{aligned}
$$

which yields

$$
\begin{aligned}
\|\hat{x}[k]\| & >\frac{\gamma_{20}}{h^{n}}\|\bar{w}\|_{\infty}-\left(1-\gamma_{x} h\right)\|z[k]\|-\frac{\gamma_{20}}{h^{n-1}}\|\bar{w}\|_{\infty} \\
\Longrightarrow\|\hat{x}[k]\|+\left(1-\gamma_{x} h\right)\|z[k]\| & >(1-h) \frac{\gamma_{20}}{h^{n}}\|\bar{w}\|_{\infty} .
\end{aligned}
$$

Observing that $\left(1-\gamma_{x} h\right)<1$ for small $h$, it is true that

$$
\begin{aligned}
\left|u_{p}[k]\right| & =\rho(\|\hat{x}[k]\|+\|z[k]\|) \\
& >\rho(1-h) \frac{\gamma_{20}}{h^{n}}\|\bar{w}\|_{\infty} .
\end{aligned}
$$


Hence, for small $h$ we can write

$$
\frac{\|\bar{w}\|_{\infty}}{\left|u_{p}[k]\right|}<\frac{1}{\rho(1-h)} \frac{h^{n}}{\gamma_{20}} .
$$

Returning to (5.35), for small $h$ we have

$$
\begin{aligned}
|\tilde{f}[k]| & \leq \gamma_{25}\left[\frac{\rho}{1+\rho} \frac{\|\hat{x}[k]\|+\left|u_{p}[k]\right|}{\left|u_{p}[k]\right|} h+\rho(1-h) \frac{1}{h^{n-1}} \frac{\|\bar{w}\|_{\infty}}{\left|u_{p}[k]\right|}\right] \\
& \leq \gamma_{25}\left[\frac{\rho}{1+\rho} \frac{\|\hat{x}[k]\|+\rho(\|\hat{x}[k]\|+\|z[k]\|)}{\rho(\|\hat{x}[k]\|+\|z[k]\|)} h+\frac{h}{\gamma_{20}}\right] \\
& \leq \gamma_{25}\left(1+\frac{1}{\gamma_{20}}\right) h .
\end{aligned}
$$

Our goal is to ensure that $\check{f}[k]$ is always projected into the interval in $\mathcal{F}$ where $f\left(\alpha\left(\bar{t}_{1}\right)\right)$ actually lies. We achieve this when $h$ is chosen small enough such that $|\tilde{f}[k]|$ is less than half the smallest distance between adjacent intervals in $\mathcal{F}$. With $d^{*}$ defined as in (4.45), if we choose $h<\frac{d^{*}}{\gamma_{25}\left(1+1 / \gamma_{20}\right)}$, then by construction, $\hat{f}[k]$ always belongs to the same interval in $\mathcal{F}$ as $f\left(\alpha\left(\bar{t}_{1}\right)\right)$. Hence, it is guaranteed to be least as close to $f\left(\alpha\left(\bar{t}_{1}\right)\right)$ as $\check{f}[k]$. Therefore, it is true that for small $h$ :

$$
\left|\hat{f}[k]-f\left(\alpha\left(\bar{t}_{1}\right)\right)\right| \leq \gamma_{25}\left(1+\frac{1}{\gamma_{20}}\right) h .
$$

We obtain an upper bound on the parameter estimation error by substituting (5.37) into (4.48):

$$
|\hat{\alpha}[k]-\alpha[k]| \leq \underbrace{\left[n \delta_{\alpha}+\frac{\gamma_{25}}{\delta_{i}}\left(1+\frac{1}{\gamma_{20}}\right)\right]}_{=: \gamma_{26}} h ;
$$

recall that this holds if (5.29) holds.

\subsection{Discrete-Time Stability Analysis}

Our goal in this section is to show that the closed loop system is stable at the sample points by using the bounds on the state and parameter estimation errors that were derived in the previous sections. The procedure will generally follow the same steps as in Section 4.3. 
In particular, the control law remains the same as in (4.2)-(4.3) and $u_{s}[k]$ is applied for $t \in\left[k T+T_{1}+T_{2}, k T+T_{1}+T_{2}+T_{3}\right)=\left[k T+T_{1}+T_{2},(k+1) T\right)$. For most of this section we will assume that the bound in (5.38) holds, which is true when (5.29) is satisfied. Hence, from now on and unless otherwise mentioned, we will assume that $k \in \mathbb{Z}^{+}$ satisfies (5.29). Also, for the purpose of this section, we assume that there are no jumps in the parameter. The case when the parameter exhibits persistent jumps is considered in Section 5.4 .

We begin by converting equations (4.2)-(4.3) to use the actual quantities instead of the estimates. Using (5.18), it is easy to see that for small $h$ we have

$$
\hat{x}[k]=x[k]+\epsilon_{x}[k], \quad \text { where }\left\|\epsilon_{x}[k]\right\| \leq \gamma_{x}\|x[k]\| h+\frac{\gamma_{20}}{h^{n-1}}\|\bar{w}\|_{\infty} .
$$

Let us examine the equation for $z[k+1]$ :

$$
\begin{aligned}
z[k+1]= & {[I+T F(\hat{\alpha}[k])] z[k]+T G(\hat{\alpha}[k]) \hat{x}[k] } \\
= & {\left[I+T\left(F(\alpha[k])+\int_{\alpha[k]}^{\hat{\alpha}[k]} \frac{d F(a)}{d a} d a\right)\right] z[k] } \\
& +T\left(G(\alpha[k])+\int_{\alpha[k]}^{\hat{\alpha}[k]} \frac{d G(a)}{d a} d a\right) x[k]+T G(\hat{\alpha}[k]) \epsilon_{x}[k] \\
= & z[k]+T G(\alpha[k]) x[k]+T F(\alpha[k]) z[k] \\
& +T\left[\int_{\alpha[k]}^{\hat{\alpha}[k]} \frac{d F(a)}{d a} d a z[k]+\int_{\alpha[k]}^{\alpha[k]} \frac{d G(a)}{d a} d a x[k]+G(\hat{\alpha}[k]) \epsilon_{x}[k]\right] .
\end{aligned}
$$

Letting

$$
e_{7}[k]:=T\left[\int_{\alpha[k]}^{\hat{\alpha}[k]} \frac{d F(a)}{d a} d a z[k]+\int_{\alpha[k]}^{\hat{\alpha}[k]} \frac{d G(a)}{d a} d a x[k]+G(\hat{\alpha}[k]) \epsilon_{x}[k]\right],
$$

we can write (5.40) compactly as

$$
z[k+1]=z[k]+T G(\alpha[k]) x[k]+T F(\alpha[k]) z[k]+e_{7}[k] .
$$

We wish to derive an upper bound on $\left\|e_{7}[k]\right\|$ : for small $h$ we have

$$
\begin{aligned}
\left\|e_{7}[k]\right\| & \leq T\left[\int_{\alpha[k]}^{\hat{\alpha}[k]}\left\|\frac{d F(a)}{d a}\right\| d a\|z[k]\|+\int_{\alpha[k]}^{\hat{\alpha}[k]}\left\|\frac{d G(a)}{d a}\right\| d a\|x[k]\|+\|G(\hat{\alpha}[k])\|\left\|\epsilon_{x}[k]\right\|\right] \\
& \leq T\left[\left(\delta_{F}\|z[k]\|+\delta_{G}\|x[k]\|\right)|\hat{\alpha}[k]-\alpha[k]|+c_{g}\left\|\epsilon_{x}[k]\right\|\right] \\
& \leq T\left[\left(\delta_{F}\|z[k]\|+\delta_{G}\|x[k]\|\right) \gamma_{26} h+c_{g}\left(\gamma_{x}\|x[k]\| h+\frac{\gamma_{20}}{h^{n-1}}\|\bar{w}\|_{\infty}\right)\right] .
\end{aligned}
$$


Substituting $h=T / N$ into the above inequality yields

$$
\left\|e_{7}[k]\right\| \leq\left[\frac{\delta_{G}}{N} \gamma_{26}+\frac{c_{g} \gamma_{x}}{N}\right] T^{2}\|x[k]\|+\frac{\delta_{F}}{N} \gamma_{26} T^{2}\|z[k]\|+\frac{c_{g} \gamma_{20} N^{n-1}}{T^{n-2}}\|\bar{w}\|_{\infty} .
$$

Hence, there exist constants $\gamma_{27}, \gamma_{28}$ and $\gamma_{29}$ such that for small $T$

$$
\left\|e_{7}[k]\right\| \leq \gamma_{27} T^{2}\|x[k]\|+\gamma_{28} T^{2}\|z[k]\|+\frac{\gamma_{29}}{T^{n-2}}\|\bar{w}\|_{\infty} .
$$

The same procedure can be carried out with the equation (4.3):

$$
\begin{aligned}
u_{s}[k]= & \frac{T}{T_{3}}[H(\hat{\alpha}[k]) z[k]+K(\hat{\alpha}[k]) \hat{x}[k]]-\frac{T_{2}}{T_{3}} u_{p}[k] \\
= & \frac{T}{T_{3}}\left[\left(H(\alpha[k])+\int_{\alpha[k]}^{\hat{\alpha}[k]} \frac{d H(a)}{d a} d a\right) z[k]\right. \\
& \left.+\left(K(\alpha[k])+\int_{\alpha[k]}^{\hat{\alpha}[k]} \frac{d K(a)}{d a} d a\right) x[k]+K(\hat{\alpha}[k]) \epsilon_{x}[k]\right]-\frac{T_{2}}{T_{3}} u_{p}[k] \\
= & \frac{T}{T_{3}}[H(\alpha[k]) z[k]+K(\alpha[k]) x[k]]-\frac{T_{2}}{T_{3}} u_{p}[k] \\
& +\frac{T}{T_{3}}\left[\int_{\alpha[k]}^{\hat{\alpha}[k]} \frac{d H(a)}{d a} d a z[k]+\int_{\alpha[k]}^{\hat{\alpha}[k]} \frac{d K(a)}{d a} d a x[k]+K(\hat{\alpha}[k]) \epsilon_{x}[k]\right] .
\end{aligned}
$$

If we define

$$
e_{8}[k]:=T\left[\int_{\alpha[k]}^{\hat{\alpha}[k]} \frac{d H(a)}{d a} d a z[k]+\int_{\alpha[k]}^{\hat{\alpha}[k]} \frac{d K(a)}{d a} d a x[k]+K(\hat{\alpha}[k]) \epsilon_{x}[k]\right],
$$

then we can write

$$
u_{s}[k]=\frac{T}{T_{3}}[H(\alpha[k]) z[k]+K(\alpha[k]) x[k]]-\frac{T_{2}}{T_{3}} u_{p}[k]+\frac{1}{T_{3}} e_{8}[k] .
$$

The upper bound on $\left\|e_{8}[k]\right\|$ is derived as follows:

$$
\begin{aligned}
\left\|e_{8}[k]\right\| & \leq T\left[\int_{\alpha[k]}^{\hat{\alpha}[k]}\left\|\frac{d H(a)}{d a}\right\| d a\|z[k]\|+\int_{\alpha[k]}^{\hat{\alpha}[k]}\left\|\frac{d K(a)}{d a}\right\| d a\|x[k]\|+\|K(\hat{\alpha}[k])\|\left\|\epsilon_{x}[k]\right\|\right] \\
& \leq T\left[\left(\delta_{H}\|z[k]\|+\delta_{K}\|x[k]\|\right)|\hat{\alpha}[k]-\alpha[k]|+c_{k}\left\|\epsilon_{x}[k]\right\|\right] \\
& \leq T\left[\left(\delta_{H}\|z[k]\|+\delta_{K}\|x[k]\|\right) \gamma_{26} h+c_{k}\left(\gamma_{x}\|x[k]\| h+\frac{\gamma_{20}}{h^{n-1}}\|\bar{w}\|_{\infty}\right)\right] \\
& =\left[\frac{\delta_{K}}{N} \gamma_{26}+\frac{c_{k} \gamma_{x}}{N}\right] T^{2}\|x[k]\|+\frac{\delta_{H}}{N} \gamma_{26} T^{2}\|z[k]\|+\frac{c_{k} \gamma_{20} N^{n-1}}{T^{n-2}}\|\bar{w}\|_{\infty} .
\end{aligned}
$$


It is easy to see that there exist constants $\gamma_{30}, \gamma_{31}$ and $\gamma_{32}$ such that for small $T$ we have

$$
\left\|e_{8}[k]\right\| \leq \gamma_{30} T^{2}\|x[k]\|+\gamma_{31} T^{2}\|z[k]\|+\frac{\gamma_{32}}{T^{n-2}}\|\bar{w}\|_{\infty}
$$

We will derive an expression for $x(t)$ when $t \in[k T,(k+1) T)$ by integrating equation $(5.1)$ :

$$
x(t)=x(k T)+\underbrace{\int_{k T}^{t} A(\alpha(\tau)) x(\tau) d \tau+\int_{k T}^{t} B(\alpha(\tau)) u_{d}(\tau) d \tau}_{=: e_{9}(t)} .
$$

In order to derive an upper bound on $\left\|e_{9}(t)\right\|$, we first consider the maximum size of the control signal in the period $[k T,(k+1) T)$. With $u_{m}[k]$ defined identically to (4.55):

$$
u_{m}[k]:=\max \left\{\left|u_{p}[k]\right|,\left|u_{s}[k]\right|\right\}
$$

we would like to examine its upper bound. Let us first consider $\left|u_{p}[k]\right|$ :

$$
\begin{aligned}
\left|u_{p}[k]\right| & =\rho(\|\hat{x}[k]\|+\|z[k]\|) \\
& \leq \rho\left[\left(1+\gamma_{x} h\right)\|x[k]\|+\|z[k]\|+\frac{\gamma_{20}}{h^{n-1}}\|\bar{w}\|_{\infty}\right] .
\end{aligned}
$$

The bound on $\left|u_{s}[k]\right|$ is derived in a similar way:

$$
\begin{aligned}
& \left|u_{s}[k]\right| \leq \frac{T}{T_{3}}[\|H(\hat{\alpha}[k])\|\|z[k]\|+\|K(\hat{\alpha}[k])\|\|\hat{x}[k]\|]+\frac{T_{2}}{T_{3}}\left|u_{p}[k]\right| \\
& \leq \frac{T}{T_{3}}\left\{c_{h}\|z[k]\|+c_{k}\left[\left(1+\gamma_{x} h\right)\|x[k]\|+\frac{\gamma_{20}}{h^{n-1}}\|\bar{w}\|_{\infty}\right]\right\} \\
& +\rho \frac{T_{2}}{T_{3}}\left[\left(1+\gamma_{x} h\right)\|x[k]\|+\|z[k]\|+\frac{\gamma_{20}}{h^{n-1}}\|\bar{w}\|_{\infty}\right] \\
& \leq\left[c_{k} \frac{T}{T_{3}}+\rho \frac{T_{2}}{T_{3}}\right]\left(1+\gamma_{x} \frac{T}{N}\right)\|x[k]\|+\left[c_{h} \frac{T}{T_{3}}+\rho \frac{T_{2}}{T_{3}}\right]\|z[k]\| \\
& +\left[c_{k} \frac{T}{T_{3}}+\rho \frac{T_{2}}{T_{3}}\right] \frac{\gamma_{20} N^{n-1}}{T^{n-1}}\|\bar{w}\|_{\infty} .
\end{aligned}
$$

It is clear from (5.46) and (5.47) that there exist constants $\gamma_{33}, \gamma_{34}$ and $\gamma_{35}$ such that for small $T$ the following holds:

$$
u_{m}[k] \leq \gamma_{33}\|x[k]\|+\gamma_{34}\|z[k]\|+\frac{\gamma_{35}}{T^{n-1}}\|\bar{w}\|_{\infty} .
$$


We will obtain a bound on $\|x(t)\|$ by applying Lemma 1 for $t \in[k T,(k+1) T)$ :

$$
\begin{aligned}
\|x(t)\| & \leq\left[\|x(k T)\|+(t-k T)\left(u_{m}[k]+\|d\|_{\infty}\right) c_{b}\right] e^{c_{a}(t-k T)} \\
& \leq\left[\|x[k]\|+T\left(\gamma_{33}\|x[k]\|+\gamma_{34}\|z[k]\|+\frac{\gamma_{35}}{T^{n-1}}\|\bar{w}\|_{\infty}+\|d\|_{\infty}\right) c_{b}\right] e^{c_{a} T} \\
& \leq\left[\left(1+c_{b} \gamma_{33} T\right)\|x[k]\|+c_{b} \gamma_{34} T\|z[k]\|+\left(\gamma_{35}+T^{n-1}\right) \frac{c_{b}}{T^{n-2}}\|\bar{w}\|_{\infty}\right] e^{c_{a} T} .
\end{aligned}
$$

It is easy to see that there exist constants $\gamma_{36}, \gamma_{37}$ and $\gamma_{38}$ such that for small $T$ we have

$$
\|x(t)\| \leq \gamma_{36}\|x[k]\|+\gamma_{37} T\|z[k]\|+\frac{\gamma_{38}}{T^{n-2}}\|\bar{w}\|_{\infty}, \quad t \in[k T,(k+1) T) .
$$

With (5.48) and (5.49) we can derive a bound on $\left\|e_{9}(t)\right\|$ - for small $T$ :

$$
\begin{aligned}
\left\|e_{9}(t)\right\| \leq & \int_{k T}^{t}\|A(\alpha(\tau))\|\|x(\tau)\| d \tau+\int_{k T}^{t}\|B(\alpha(\tau))\|\left(|u(\tau)|+\|d\|_{\infty}\right) d \tau \\
\leq & c_{a}\left[\gamma_{36}\|x[k]\|+\gamma_{37} T\|z[k]\|+\frac{\gamma_{38}}{T^{n-2}}\|\bar{w}\|_{\infty}\right](t-k T) \\
& +c_{b}\left[\gamma_{33}\|x[k]\|+\gamma_{34}\|z[k]\|+\frac{\gamma_{35}}{T^{n-1}}\|\bar{w}\|_{\infty}+\|d\|_{\infty}\right](t-k T) \\
\leq & {\left[\left(c_{a} \gamma_{36}+c_{b} \gamma_{33}\right)\|x[k]\|+\left(c_{a} \gamma_{37} T+c_{b} \gamma_{34}\right)\|z[k]\|\right.} \\
& \left.+\left(c_{a} \gamma_{38} T+c_{b} \gamma_{35}+c_{b} T^{n-1}\right) \frac{1}{T^{n-1}}\|\bar{w}\|_{\infty}\right](t-k T) .
\end{aligned}
$$

Clearly, for small $T$, there exist constants $\gamma_{39}, \gamma_{40}$ and $\gamma_{41}$ so that

$$
\left\|e_{9}(t)\right\| \leq\left[\gamma_{39}\|x[k]\|+\gamma_{40}\|z[k]\|+\frac{\gamma_{41}}{T^{n-1}}\|\bar{w}\|_{\infty}\right](t-k T), \quad t \in[k T,(k+1) T) .
$$

We can now discretize the plant state equation with the control signal applied; using (5.45) yields

$$
\begin{aligned}
x((k+1) T)= & x(k T)+\int_{k T}^{(k+1) T} A(\alpha(\tau)) x(\tau) d \tau+\int_{k T}^{(k+1) T} B(\alpha(\tau)) u_{d}(\tau) d \tau \\
= & x(k T)+\int_{k T}^{(k+1) T}\left(A(\alpha(k T))+\int_{k T}^{\tau} \dot{A}(\alpha(\theta)) d \theta\right) x(k T) d \tau \\
& +\int_{k T}^{(k+1) T} A(\alpha(\tau)) e_{9}(\tau) d \tau+\int_{k T}^{(k+1) T} B(\alpha(\tau)) d(\tau) d \tau \\
& \left.+\int_{k T+T_{1}}^{k T+T_{1}+T_{2}}\left(B(\alpha(k T))+\int_{k T}^{\tau} \dot{B}(\alpha(\theta)) d \theta\right)\right) u_{p}[k] d \tau \\
& \left.+\int_{k T+T_{1}+T_{2}}^{k T+T_{1}+T_{2}+T_{3}}\left(B(\alpha(k T))+\int_{k T}^{\tau} \dot{B}(\alpha(\theta)) d \theta\right)\right) u_{s}[k] d \tau .
\end{aligned}
$$


After re-arranging the terms and using our standard notation of $x[k]=x(k T)$ we obtain the following:

$$
\begin{aligned}
x[k+1]= & x[k]+T A(\alpha[k]) x[k]+T_{2} B(\alpha[k]) u_{p}[k]+T_{3} B(\alpha[k]) u_{s}[k] \\
& +\int_{k T}^{(k+1) T} \int_{k T}^{\tau} \dot{A}(\alpha(\theta)) d \theta x[k] d \tau+\int_{k T}^{(k+1) T} A(\alpha(\tau)) e_{9}(\tau) d \tau \\
& +\int_{k T}^{(k+1) T} B(\alpha(\tau)) d(\tau) d \tau+\int_{k T+T_{1}}^{k T+T_{1}+T_{2}} \int_{k T}^{\tau} \dot{B}(\alpha(\theta)) d \theta u_{p}[k] d \tau \\
& +\int_{k T+T_{1}+T_{2}}^{k T+T_{1}+T_{2}+T_{3}} \int_{k T}^{\tau} \dot{B}(\alpha(\theta)) d \theta u_{s}[k] d \tau .
\end{aligned}
$$

We define a new error term:

$$
\begin{aligned}
e_{10}[k]:= & \int_{k T}^{(k+1) T} \int_{k T}^{\tau} \dot{A}(\alpha(\theta)) d \theta x[k] d \tau+\int_{k T}^{(k+1) T} A(\alpha(\tau)) e_{9}(\tau) d \tau \\
& +\int_{k T}^{(k+1) T} B(\alpha(\tau)) d(\tau) d \tau+\int_{k T+T_{1}}^{k T+T_{1}+T_{2}} \int_{k T}^{\tau} \dot{B}(\alpha(\theta)) d \theta u_{p}[k] d \tau \\
& +\int_{k T+T_{1}+T_{2}}^{k T+T_{1}+T_{2}+T_{3}} \int_{k T}^{\tau} \dot{B}(\alpha(\theta)) d \theta u_{s}[k] d \tau,
\end{aligned}
$$

and substitute (5.43) in place of $u_{s}[k]$ to obtain the following discretized system:

$$
\begin{aligned}
x[k+1]= & x[k]+T A(\alpha[k]) x[k]+T_{2} B(\alpha[k]) u_{p}[k] \\
& +T_{3} B(\alpha[k])\left(\frac{T}{T_{3}}[H(\alpha[k]) z[k]+K(\alpha[k]) x[k]]-\frac{T_{2}}{T_{3}} u_{p}[k]+\frac{1}{T_{3}} e_{8}[k]\right)+e_{10}[k] \\
= & x[k]+T[A(\alpha[k])+B(\alpha[k]) K(\alpha[k])] x[k]+T B(\alpha[k]) H(\alpha[k]) z[k] \\
& +\underbrace{B(\alpha[k]) e_{8}[k]+e_{10}[k]}_{=: e_{11}[k]} .
\end{aligned}
$$

We will proceed to derive an upper bound on $\left\|e_{10}[k]\right\|$ : for small $T$ we have

$$
\begin{aligned}
\left\|e_{10}[k]\right\| \leq & \int_{k T}^{(k+1) T} \int_{k T}^{\tau}\|\dot{A}(\alpha(\theta))\| d \theta\|x[k]\| d \tau+\int_{k T}^{(k+1) T}\|A(\alpha(\tau))\|\left\|e_{9}(\tau)\right\| d \tau \\
& +\int_{k T}^{(k+1) T}\|B(\alpha(\tau))\|\|d(\tau)\| d \tau+\int_{k T+T_{1}}^{k T+T_{1}+T_{2}} \int_{k T}^{\tau}\|\dot{B}(\alpha(\theta))\| d \theta\left|u_{p}[k]\right| d \tau \\
& +\int_{k T+T_{1}+T_{2}}^{k T+T_{1}+T_{2}+T_{3}} \int_{k T}^{\tau}\|\dot{B}(\alpha(\theta))\| d \theta\left|u_{s}[k]\right| d \tau,
\end{aligned}
$$


Using (5.50) to bound $\left\|e_{9}(\tau)\right\|$ and (5.48) to bound $\left|u_{p}[k]\right|$ and $\left|u_{s}[k]\right|$ yields

$$
\begin{aligned}
\left\|e_{10}[k]\right\| \leq & \int_{k T}^{(k+1) T} \delta_{A} \delta_{\alpha}\|x[k]\|(\tau-k T) d \tau+c_{b} \int_{k T}^{(k+1) T}\|\bar{w}\|_{\infty} d \tau \\
& +c_{a} \int_{k T}^{(k+1) T}\left[\gamma_{39}\|x[k]\|+\gamma_{40}\|z[k]\|+\frac{\gamma_{41}}{T^{n-1}}\|\bar{w}\|_{\infty}\right](\tau-k T) d \tau \\
& +\int_{k T+T_{1}}^{k T+T_{1}+T_{2}+T_{3}} \delta_{B} \delta_{\alpha}\left[\gamma_{33}\|x[k]\|+\gamma_{34}\|z[k]\|+\frac{\gamma_{35}}{T^{n-1}}\|\bar{w}\|_{\infty}\right](\tau-k T) d \tau \\
\leq & \delta_{A} \delta_{\alpha}\|x[k]\| \frac{T^{2}}{2}+c_{b}\|\bar{w}\|_{\infty} T+c_{a}\left[\gamma_{39}\|x[k]\|+\gamma_{40}\|z[k]\|+\frac{\gamma_{41}}{T^{n-1}}\|\bar{w}\|_{\infty}\right] \frac{T^{2}}{2} \\
& +\delta_{B} \delta_{\alpha}\left[\gamma_{33}\|x[k]\|+\gamma_{34}\|z[k]\|+\frac{\gamma_{35}}{T^{n-1}}\|\bar{w}\|_{\infty}\right] \frac{T^{2}}{2}\left(1-\frac{T_{1}^{2}}{T^{2}}\right) .
\end{aligned}
$$

It is easy to see that there exist constants $\gamma_{42}, \gamma_{43}$ and $\gamma_{44}$ such that for small $T$ we have

$$
\left\|e_{10}[k]\right\| \leq \gamma_{42} T^{2}\|x[k]\|+\gamma_{43} T^{2}\|z[k]\|+\frac{\gamma_{44}}{T^{n-3}}\|\bar{w}\|_{\infty}
$$

The bound on $\left\|e_{11}[k]\right\|$ is calculated using results from (5.44) and (5.52):

$$
\begin{aligned}
\left\|e_{11}[k]\right\| & \leq\|B(\alpha[k])\|\left\|e_{8}[k]\right\|+\left\|e_{10}[k]\right\| \\
& \leq\left[c_{b} \gamma_{30}+\gamma_{42}\right] T^{2}\|x[k]\|+\left[c_{b} \gamma_{31}+\gamma_{43}\right] T^{2}\|z[k]\|+\left[\gamma_{32}+\gamma_{44} T\right] \frac{1}{T^{n-2}}\|\bar{w}\|_{\infty} .
\end{aligned}
$$

Hence, there exist constants $\gamma_{45}, \gamma_{46}$ and $\gamma_{47}$ such that for small $T$ the following holds:

$$
\left\|e_{11}[k]\right\| \leq \gamma_{45} T^{2}\|x[k]\|+\gamma_{46} T^{2}\|z[k]\|+\frac{\gamma_{47}}{T^{n-2}}\|\bar{w}\|_{\infty}
$$

We can now proceed with the analysis of the closed loop system equation, which is formed by combining (5.41) and (5.51):

$$
\left[\begin{array}{c}
x[k+1] \\
z[k+1]
\end{array}\right]=\left[\begin{array}{c}
x[k] \\
z[k]
\end{array}\right]+T\left[\begin{array}{cc}
A(\alpha[k])+B(\alpha[k]) K(\alpha[k]) & B(\alpha[k]) H(\alpha[k]) \\
G(\alpha[k]) & F(\alpha[k])
\end{array}\right]\left[\begin{array}{c}
x[k] \\
z[k]
\end{array}\right]+\left[\begin{array}{c}
e_{11}[k] \\
e_{7}[k]
\end{array}\right] .
$$

The closed loop state and the discretization error are defined as follows:

$$
\xi[k]:=\left[\begin{array}{l}
x[k] \\
z[k]
\end{array}\right] \quad \text { and } \quad e_{12}[k]:=\left[\begin{array}{c}
e_{11}[k] \\
e_{7}[k]
\end{array}\right]
$$

which results in the closed loop equation of the form

$$
\xi[k+1]=\left(I+T\left[\begin{array}{cc}
A(\alpha[k])+B(\alpha[k]) K(\alpha[k]) & B(\alpha[k]) H(\alpha[k]) \\
G(\alpha[k]) & F(\alpha[k])
\end{array}\right]\right) \xi[k]+e_{12}[k] .
$$


We derive an upper bound on $\left\|e_{12}[k]\right\|$ by using (5.42) and (5.53):

$$
\begin{aligned}
\left\|e_{12}[k]\right\| & =\left\|e_{7}[k]\right\|+\left\|e_{11}[k]\right\| \\
& \leq\left[\gamma_{27}+\gamma_{45}\right] T^{2}\|x[k]\|+\left[\gamma_{28}+\gamma_{46}\right] T^{2}\|z[k]\|+\left[\gamma_{29}+\gamma_{47}\right] \frac{1}{T^{n-2}}\|\bar{w}\|_{\infty} .
\end{aligned}
$$

With $\|\xi[k]\|=\|x[k]\|+\|z[k]\|$, it follows that there exist constants $\gamma_{48}$ and $\gamma_{49}$ such that for small $T$

$$
\left\|e_{12}[k]\right\| \leq \gamma_{48} T^{2}\|\xi[k]\|+\frac{\gamma_{49}}{T^{n-2}}\|\bar{w}\|_{\infty} .
$$

An important observation is made about the closed loop system by considering (3.25):

$$
\left[\begin{array}{cc}
A(\alpha[k])+B(\alpha[k]) K(\alpha[k]) & B(\alpha[k]) H(\alpha[k]) \\
G(\alpha[k]) & F(\alpha[k])
\end{array}\right]=\left[\begin{array}{c}
X \\
Z
\end{array}\right] P(\alpha[k])\left[\begin{array}{c}
X \\
Z
\end{array}\right]^{-1} .
$$

Substituting the above into (5.54) yields

$$
\xi[k+1]=\left(I+T\left[\begin{array}{c}
X \\
Z
\end{array}\right] P(\alpha[k])\left[\begin{array}{c}
X \\
Z
\end{array}\right]^{-1}\right) \xi[k]+e_{12}[k] .
$$

Defining the following transformed variables:

$$
\bar{\xi}[k]:=\left[\begin{array}{c}
X \\
Z
\end{array}\right]^{-1} \xi[k] \quad \text { and } \quad \bar{e}_{12}[k]:=\left[\begin{array}{c}
X \\
Z
\end{array}\right]^{-1} e_{12}[k],
$$

(5.57) accordingly becomes

$$
\bar{\xi}[k+1]=[I+T P(\alpha[k])] \bar{\xi}[k]+\bar{e}_{12}[k],
$$

with

$$
\begin{aligned}
\left\|\bar{e}_{12}[k]\right\| & \leq\left\|\left[\begin{array}{c}
X \\
Z
\end{array}\right]^{-1}\right\|\left\|e_{12}[k]\right\| \\
& \leq\left\|\left[\begin{array}{c}
X \\
Z
\end{array}\right]^{-1}\right\|\left(\gamma_{48} T^{2}\|\xi[k]\|+\frac{\gamma_{49}}{T^{n-2}}\|\bar{w}\|_{\infty}\right) \\
& \leq \underbrace{\left\|\left[\begin{array}{c}
X \\
Z
\end{array}\right]^{-1}\right\|\left\|\left[\begin{array}{c}
X \\
Z
\end{array}\right]\right\| \gamma_{48} T^{2}\|\bar{\xi}[k]\|+\underbrace{\left\|\left[\begin{array}{c}
X \\
Z
\end{array}\right]^{-1}\right\| \gamma_{49}}_{=: \gamma_{50}} \frac{1}{T^{n-2}}\|\bar{w}\|_{\infty}]}_{\gamma_{16}}
\end{aligned}
$$


By Proposition 1 there exist $\bar{T}>0$ and $\bar{\lambda}>0$ so that for all $T \in(0, \bar{T})$ and $\lambda \in(0, \bar{\lambda})$ we have

$$
\|I+T P(\alpha[k])\| \leq 1-\lambda T \text { for all } \alpha[k] \in \mathcal{A} .
$$

With this result we can establish a bound on $\|\bar{\xi}[k+1]\|$ by taking the norms of both sides of (5.59); fix $\lambda \in(0, \bar{\lambda})$ and let $T \in(0, \bar{T})$ be arbitrary, so that

$$
\begin{aligned}
\|\bar{\xi}[k+1]\| & \leq\|I+T P(\alpha[k])\|\|\bar{\xi}[k]\|+\left\|\bar{e}_{12}[k]\right\| \\
& \leq(1-\lambda T)\|\bar{\xi}[k]\|+\gamma_{16} \gamma_{48} T^{2}\|\bar{\xi}[k]\|+\frac{\gamma_{50}}{T^{n-2}}\|\bar{w}\|_{\infty} .
\end{aligned}
$$

Similarly to the noise free case, we would like to find a $T^{*} \in(0, \bar{T})$ such that for all $T \in\left(0, T^{*}\right)$ we have

$$
\gamma_{16} \gamma_{48} T^{2} \leq \frac{\lambda}{2} T
$$

Clearly, we can choose

$$
T^{*}=\min \left\{\frac{\lambda}{2 \gamma_{16} \gamma_{48}}, \frac{\bar{T}}{2}\right\} .
$$

Therefore, using (5.61) in (5.60), we derive the following bound on $\|\bar{\xi}[k+1]\|$ which holds for small $T$ :

$$
\begin{aligned}
\|\bar{\xi}[k+1]\| & \leq(1-\lambda T)\|\bar{\xi}[k]\|+\frac{\lambda}{2} T\|\bar{\xi}[k]\|+\frac{\gamma_{50}}{T^{n-2}}\|\bar{w}\|_{\infty} \\
& \leq\left(1-\frac{\lambda}{2} T\right)\|\bar{\xi}[k]\|+\frac{\gamma_{50}}{T^{n-2}}\|\bar{w}\|_{\infty} \\
& \leq e^{-\frac{\lambda}{2} T}\|\bar{\xi}[k]\|+\frac{\gamma_{50}}{T^{n-2}}\|\bar{w}\|_{\infty} .
\end{aligned}
$$

Up until this point we have only considered those $k$ for which condition (5.29) was satisfied. If it is not, i.e. $\left\|\left[\begin{array}{l}x[k] \\ z[k]\end{array}\right]\right\| \leq \frac{\gamma_{20}}{\left(1-\gamma_{x} h\right) h^{n}}\|\bar{w}\|_{\infty}$, then an appropriate upper bound on $\left\|\left[\begin{array}{l}x[k+1] \\ z[k+1]\end{array}\right]\right\|$ must be derived. We will first bound $\|x[k+1]\|$ using (5.49):

$$
\| x\left[k+1\left\|\leq \gamma_{36}\right\| x[k]\left\|+\gamma_{37} T\right\| z[k]\left\|+\frac{\gamma_{38}}{T^{n-2}}\right\| \bar{w} \|_{\infty} .\right.
$$

Now we will derive a bound on $\|z[k+1]\|$ by taking the norms of both sides of (4.2): using (5.39) yields

$$
\begin{aligned}
\|z[k+1]\| & \leq\|I+T F(\hat{\alpha}[k])\|\|z[k]\|+\|T G(\hat{\alpha}[k])\|\|\hat{x}[k]\| \\
& \leq[1+T\|F(\hat{\alpha}[k])\|]\|z[k]\|+T\|G(\hat{\alpha}[k])\|\left[\left(1+\gamma_{x} h\right)\|x[k]\|+\frac{\gamma_{20}}{h^{n-1}}\|\bar{w}\|_{\infty}\right] \\
& \leq c_{g}\left(1+\gamma_{x} \frac{T}{N}\right) T\|x[k]\|+\left[1+c_{f} T\right]\|z[k]\|+\frac{c_{g} \gamma_{20} N^{n-1}}{T^{n-2}}\|\bar{w}\|_{\infty} .
\end{aligned}
$$


Combining (5.64) and (5.65) we obtain

$$
\begin{aligned}
\left\|\left[\begin{array}{l}
x[k+1]] \\
z[k+1]
\end{array}\right]\right\| & \| x[k+1\|+\| z[k+1] \| \\
\leq & {\left[\gamma_{36}+c_{g}\left(1+\gamma_{x} \frac{T}{N}\right) T\right]\|x[k]\|+\left[1+\left(\gamma_{37}+c_{f}\right) T\right]\|z[k]\| } \\
& +\frac{\gamma_{38}+c_{g} \gamma_{20} N^{n-1}}{T^{n-2}}\|\bar{w}\|_{\infty} .
\end{aligned}
$$

Hence, there exist constants $\gamma_{51}$ and $\gamma_{52}$ such that for small $T$ we have

$$
\begin{aligned}
\left\|\left[\begin{array}{l}
x[k+1] \\
z[k+1]
\end{array}\right]\right\| & \leq \gamma_{51}\left\|\left[\begin{array}{l}
x[k] \\
z[k]
\end{array}\right]\right\|+\frac{\gamma_{52}}{T^{n-2}}\|\bar{w}\|_{\infty} \\
& \leq \gamma_{51} \frac{\gamma_{20}}{\left(1-\gamma_{x} h\right) h^{n}}\|\bar{w}\|_{\infty}+\frac{\gamma_{52}}{T^{n-2}}\|\bar{w}\|_{\infty} \\
& \leq\left[\frac{\gamma_{20} \gamma_{51} N^{n}}{\left(1-\gamma_{x} T / N\right)}+\gamma_{52} T^{2}\right] \frac{1}{T^{n}}\|\bar{w}\|_{\infty} .
\end{aligned}
$$

It is easy to see that for small $T$ there exists a constant $\gamma_{53}$ such that

$$
\|\xi[k+1]\| \leq \frac{\gamma_{53}}{T^{n}}\|\bar{w}\|_{\infty}
$$

and so

$$
\begin{aligned}
\|\bar{\xi}[k+1]\| & \leq\left\|\left[\begin{array}{c}
X \\
Z
\end{array}\right]^{-1}\right\|\|\xi[k+1]\| \\
& \leq \underbrace{\left\|\left[\begin{array}{c}
X \\
Z
\end{array}\right]^{-1}\right\| \gamma_{53} \frac{1}{T^{n}}\|\bar{w}\|_{\infty} .}_{=: \gamma_{54}}
\end{aligned}
$$

Combining (5.63) and (5.66) we have

$$
\|\bar{\xi}[k+1]\| \leq e^{-\frac{\lambda}{2} T}\|\bar{\xi}[k]\|+\left[\gamma_{50} T^{2}+\gamma_{54}\right] \frac{1}{T^{n}}\|\bar{w}\|_{\infty}, \quad k \in \mathbb{Z}^{+} .
$$

Clearly, there exists a constant $\gamma_{55}$ such that for small $T$ the following holds:

$$
\|\bar{\xi}[k+1]\| \leq e^{-\frac{\lambda}{2} T}\|\bar{\xi}[k]\|+\frac{\gamma_{55}}{T^{n}}\|\bar{w}\|_{\infty}, \quad k \in \mathbb{Z}^{+} .
$$

We can use this result to derive an upper bound for $\|\bar{\xi}[k]\|$ :

$$
\|\bar{\xi}[k]\| \leq e^{-\frac{\lambda}{2} k T}\|\bar{\xi}[0]\|+\left(1+e^{-\frac{\lambda}{2} T}+\ldots+e^{-\frac{\lambda}{2}(k-1) T}\right) \frac{\gamma_{55}}{T^{n}}\|\bar{w}\|_{\infty}, \quad k \in \mathbb{Z}^{+}
$$


which can be simplified using the formula for the sum of the geometric progression:

$$
\begin{aligned}
\|\bar{\xi}[k]\| & \leq e^{-\frac{\lambda}{2} k T}\|\bar{\xi}[0]\|+\frac{1-e^{-\frac{\lambda}{2} k T}}{1-e^{-\frac{\lambda}{2} T}} \frac{\gamma_{55}}{T^{n}}\|\bar{w}\|_{\infty} \\
& \leq e^{-\frac{\lambda}{2} k T}\|\bar{\xi}[0]\|+\frac{\gamma_{55}}{1-e^{-\frac{\lambda}{2} T}} \frac{1}{T^{n}}\|\bar{w}\|_{\infty}, \quad k \in \mathbb{Z}^{+} .
\end{aligned}
$$

It is easy to see that there exists $c>0$ such that for small $T$ we have: $e^{-\frac{\lambda}{2} T} \leq 1-c T$. Consequently,

$$
\|\bar{\xi}[k]\| \leq e^{-\frac{\lambda}{2} k T}\|\bar{\xi}[0]\|+\frac{\gamma_{55}}{c} \frac{1}{T^{n+1}}\|\bar{w}\|_{\infty}, \quad k \in \mathbb{Z}^{+} .
$$

We have, therefore, proven the following proposition:

Proposition 4. Given a system of the form (3.26)-(3.27) satisfying Assumptions $1-6$ and a controller of the form (4.2)-(4.3) satisfying Assumption 7, there exists a constant $\gamma_{56}$ and $T^{\prime}>0$ so that for all $T \in\left(0, T^{\prime}\right), x(0) \in \mathbb{R}^{n}, z(0) \in \mathbb{R}^{m-n}, \alpha \in A C(\mathcal{A}), w \in P C(\mathbb{R})$, and $d \in P C(\mathbb{R})$ we have

$$
\|\bar{\xi}[k]\| \leq e^{-\frac{\lambda}{2} k T}\|\bar{\xi}[0]\|+\frac{\gamma_{56}}{T^{n+1}}\|\bar{w}\|_{\infty}, \quad k \in \mathbb{Z}^{+}
$$

\subsection{Handling Parameter Jumps}

In this section we will extend the result from Proposition 4 to show that the closed loop system is stable even when there are jumps in the parameter. We begin by introducing the following continuous time quantities:

$$
\begin{gathered}
z(t):=z[k] \quad \text { for } \quad t \in[k T,(k+1) T), \\
\xi(t):=\left[\begin{array}{l}
x(t) \\
z(t)
\end{array}\right]
\end{gathered}
$$

Theorem 2. Given a system of the form (3.26)-(3.27) satisfying Assumptions $1-6$ and a controller of the form (4.2)-(4.3) satisfying Assumption 7, there exists constants $\gamma_{74}$ and $\gamma_{75}$ and $T^{\prime}>0$ so that for all $T \in\left(0, T^{\prime}\right), x(0) \in \mathbb{R}^{n}, z(0) \in \mathbb{R}^{m-n}, \alpha \in P S\left(\mathcal{A}, T_{0}\right)$, $w \in P C(\mathbb{R})$, and $d \in P C(\mathbb{R})$ we have

$$
\|\xi(t)\| \leq \gamma_{75} e^{-\frac{\lambda}{4} t}\|\xi(0)\|+\frac{\gamma_{74}}{T^{n+1}}\|\bar{w}\|_{\infty}, \quad t \in \mathbb{R}^{+} .
$$


Proof. Let $\underline{k}, \bar{k} \in \mathbb{N}$ be such that in the interval $t \in[\underline{k} T, \bar{k} T]$ there are no discontinuities in $\alpha(t)$. Then from Proposition 4 it follows immediately that

$$
\|\bar{\xi}[k]\| \leq e^{-\frac{\lambda}{2}(k-\underline{k}) T}\|\bar{\xi}[\underline{k}]\|+\frac{\gamma_{56}}{T^{n+1}}\|\bar{w}\|_{\infty}, \quad k \in\{\underline{k}, \ldots, \bar{k}\} .
$$

Since we now allow for an occasional parameter jump, we will need to introduce appropriate notation. Recall that $\alpha \in P S\left(\mathcal{A}, T_{0}\right)$ implies that there is a lower bound $T_{0}$ on the time between parameter jumps. Throughout our analysis we have assumed that $t_{0}=0$; hence, we let $\left\{t_{i}: i \in \mathbb{N}\right\}$ denote a sequence of times satisfying

- $t_{1} \geq 0$, and $t_{1}=0$ if $\alpha(t)$ is discontinuous at $t=0$;

- $t_{i+1}-t_{i} \geq T_{0}, \quad i \in \mathbb{N}$

- $\alpha(t)$ is continuous on $\left(t_{i}, t_{i+1}\right), \quad i \in \mathbb{Z}^{+}$.

We let these times represent the instances where the discontinuity in $\alpha(t)$ occurs; notice that the sequence is not unique in general. Now, for a given sampling period $T$, we would like to identify the periods where $\alpha(t)$ loses continuity. To this end, we define $k_{0}(T):=0$ and

$$
k_{i}(T):=\left\lfloor\frac{t_{i}}{T}\right\rfloor, \quad i \in \mathbb{N}
$$

using these we have that all the discontinuities are contained in $\cup_{i=0}^{\infty}\left[k_{i}(T) T,\left(k_{i}(T)+1\right) T\right)$. Consequently, $\alpha(t)$ is absolutely continuous on $\cup_{i=0}^{\infty}\left[\left(k_{i}(T)+1\right) T, k_{i+1}(T) T\right)$. Hence, using (5.68), for small $T$ we have

$$
\begin{gathered}
\|\bar{\xi}[k]\| \leq e^{-\frac{\lambda}{2}\left(k-k_{i}(T)-1\right) T}\left\|\bar{\xi}\left[k_{i}(T)+1\right]\right\|+\frac{\gamma_{56}}{T^{n+1}}\|\bar{w}\|_{\infty}, \\
k \in\left\{k_{i}(T)+1, \ldots, k_{i+1}(T)\right\}, i \in \mathbb{Z}^{+} .
\end{gathered}
$$

So far we have proven nice properties for $\bar{\xi}[k]$ when there are no parameter jumps. We would like to show that the size of $\bar{\xi}[k]$ increases by a bounded amount in the periods when there is a jump. We begin by investigating the behaviour of $x[k]$ : by integrating the state equation we have

$$
\begin{aligned}
& x((k+1) T)=x(k T)+\int_{k T}^{(k+1) T} A(\alpha(\tau)) x(\tau) d \tau+\int_{k T}^{(k+1) T} B(\alpha(\tau)) u(\tau) \\
& \Longrightarrow\|x[k+1]-x[k]\| \leq \int_{k T}^{(k+1) T}\|A(\alpha(\tau))\|\|x(\tau)\| d \tau+\int_{k T}^{(k+1) T}\|B(\alpha(\tau))\||u(\tau)| d \tau .
\end{aligned}
$$


It turns out that the bounds (5.48) and (5.49) hold even when there is a discontinuity in $\alpha(t)$; plugging them into the above yields

$$
\begin{aligned}
\|x[k+1]-x[k]\| \leq & \left(c_{a} \gamma_{36}+c_{b} \gamma_{33}\right) T\|x[k]\|+\left(c_{a} \gamma_{37}+c_{b} \gamma_{34}\right) T\|z[k]\| \\
& +\frac{c_{a} \gamma_{38} T+c_{b} \gamma_{35}}{T^{n-2}}\|\bar{w}\|_{\infty} .
\end{aligned}
$$

Clearly there exist constants $\gamma_{57}, \gamma_{58}$ and $\gamma_{59}$ such that for small $T$ the following holds:

$$
\|x[k+1]-x[k]\| \leq \gamma_{57} T\|x[k]\|+\gamma_{58} T\|z[k]\|+\frac{\gamma_{59}}{T^{n-2}}\|\bar{w}\|_{\infty} .
$$

We derive a bound $\|z[k+1]-z[k]\|$ from equation (4.3) in a similar fashion:

$$
\begin{aligned}
\|z[k+1]-z[k]\| & \leq T\|F(\hat{\alpha}[k])\| z[k]+T\|G(\hat{\alpha}[k])\|\|\hat{x}[k]\| \\
& \leq T c_{f}\|z[k]\|+T c_{g}\left[\left(1+\gamma_{x} \frac{T}{N}\right)\|x[k]\|+\frac{\gamma_{20} N^{n-1}}{T^{n-1}}\|\bar{w}\|_{\infty}\right] .
\end{aligned}
$$

It is easy to see that there exist constants $\gamma_{60}, \gamma_{61}$ and $\gamma_{62}$ so that for small $T$ we have

$$
\|z[k+1]-z[k]\| \leq \gamma_{60} T\|x[k]\|+\gamma_{61} T\|z[k]\|+\frac{\gamma_{62}}{T^{n-2}}\|\bar{w}\|_{\infty} .
$$

Therefore, by combining the two inequalities we obtain a bound on $\|\xi[k+1]-\xi[k]\|$ for all $k \in \mathbb{Z}^{+}$:

$$
\begin{aligned}
\|\xi[k+1]-\xi[k]\| & =\left\|\left[\begin{array}{l}
x[k+1]-x[k]] \\
z[k+1]-z[k]
\end{array}\right]\right\| \\
& =\|x[k+1]-x[k]\|+\|z[k+1]-z[k]\| \\
& \leq\left(\gamma_{57}+\gamma_{60}\right) T\|x[k]\|+\left(\gamma_{58}+\gamma_{61}\right) T\|z[k]\|+\frac{\gamma_{59}+\gamma_{62}}{T^{n-2}}\|\bar{w}\|_{\infty} .
\end{aligned}
$$

Obviously, there exist constants $\gamma_{63}$ and $\gamma_{64}$ so that for small $T$

$$
\|\xi[k+1]-\xi[k]\| \leq \gamma_{63} T\|\xi[k]\|+\frac{\gamma_{64}}{T^{n-2}}\|\bar{w}\|_{\infty}, \quad k \in \mathbb{Z}^{+} .
$$

We now convert the above equation to use the transformed state variable:

$$
\begin{aligned}
& \|\bar{\xi}[k+1]-\bar{\xi}[k]\| \leq\left\|\left[\begin{array}{c}
X \\
Z
\end{array}\right]^{-1}\right\|\|\xi[k+1]-\xi[k]\| \\
& \leq\left\|\left[\begin{array}{c}
X \\
Z
\end{array}\right]^{-1}\right\|\left[\gamma_{63} T\|\xi[k]\|+\frac{\gamma_{64}}{T^{n-2}}\|\bar{w}\|_{\infty}\right] \\
& \leq \underbrace{\left\|\left[\begin{array}{c}
X \\
Z
\end{array}\right]^{-1}\right\|\left\|\left[\begin{array}{c}
X \\
Z
\end{array}\right]\right\| \gamma_{63} T\|\bar{\xi}[k]\|}_{=: \gamma_{65}}+\underbrace{\left\|\left[\begin{array}{c}
X \\
Z
\end{array}\right]^{-1}\right\| \gamma_{64}}_{=: \gamma_{66}} \frac{1}{T^{n-2}}\|\bar{w}\|_{\infty}, \quad k \in \mathbb{Z}^{+},
\end{aligned}
$$


which yields the following result:

$$
\|\bar{\xi}[k+1]\| \leq\left(1+\gamma_{65} T\right)\|\bar{\xi}[k]\|+\frac{\gamma_{66}}{T^{n-2}}\|\bar{w}\|_{\infty}, \quad k \in \mathbb{Z}^{+} .
$$

By combining (5.69) and (5.73) we have

$$
\begin{aligned}
\|\bar{\xi}[k]\| \leq & e^{-\frac{\lambda}{2}\left(k-k_{i}(T)-1\right) T}\left[\left(1+\gamma_{65} T\right)\left\|\bar{\xi}\left[k_{i}(T)\right]\right\|+\frac{\gamma_{66}}{T^{n-2}}\|\bar{w}\|_{\infty}\right]+\frac{\gamma_{56}}{T^{n+1}}\|\bar{w}\|_{\infty} \\
\leq & \left(1+\gamma_{65} T\right) e^{-\frac{\lambda}{2}\left(k-k_{i}(T)-1\right) T}\left\|\bar{\xi}\left[k_{i}(T)\right]\right\|+\frac{\gamma_{66} T^{3}+\gamma_{56}}{T^{n+1}}\|\bar{w}\|_{\infty}, \\
& \quad k \in\left\{k_{i}(T), \ldots, k_{i+1}(T)\right\}, i \in \mathbb{Z}^{+} .
\end{aligned}
$$

Hence, there exists a constant $\gamma_{67} \geq \gamma_{56}$ such that for small $T$ we have

$$
\begin{gathered}
\|\bar{\xi}[k]\| \leq\left(1+\gamma_{65} T\right) e^{-\frac{\lambda}{2}\left(k-k_{i}(T)-1\right) T}\left\|\bar{\xi}\left[k_{i}(T)\right]\right\|+\frac{\gamma_{67}}{T^{n+1}}\|\bar{w}\|_{\infty}, \\
k \in\left\{k_{i}(T), \ldots, k_{i+1}(T)\right\}, i \in \mathbb{Z}^{+} .
\end{gathered}
$$

Now we would like to show that $\|\bar{\xi}[k]\|$ decreases at $k=k_{i}(T)$ for all $i \in \mathbb{Z}^{+}$. To this end, we define

$$
\bar{k}_{i}(T):=\left\lfloor\frac{t_{i}+\frac{T_{0}}{2}}{T}\right\rfloor
$$

and note that

$$
\limsup _{T \rightarrow 0}\left[\left(\bar{k}_{i}(T)-k_{i}(T)\right) T\right]=\frac{T_{0}}{2}, \quad i \in \mathbb{N} .
$$

Claim 1. There exists a $\tilde{T} \in\left(0, T^{\prime}\right)$ such that for all $T \in(0, \tilde{T})$

$$
\left(1+\gamma_{65} T\right) e^{-\frac{\lambda}{2}\left(k-k_{i}(T)-1\right) T} \leq e^{-\frac{\lambda}{3}\left(k-k_{i}(T)\right) T}, \quad k \in\left\{\bar{k}_{i}(T), \ldots, k_{i+1}(T)\right\}, i \in \mathbb{N} .
$$

Proof. After re-arranging we have

$$
\begin{aligned}
\left(1+\gamma_{65} T\right) e^{\frac{\lambda}{2} T} & \leq e^{-\frac{\lambda}{3}\left(k-k_{i}(T)\right) T} e^{\frac{\lambda}{2}\left(k-k_{i}(T)\right) T} \\
& =e^{\frac{\lambda}{6}\left(k-k_{i}(T)\right) T}
\end{aligned}
$$

It is easy to see that

$$
\limsup _{T \rightarrow 0}\left[\left(1+\gamma_{65} T\right) e^{\frac{\lambda}{2} T}\right]=1
$$

also, using (5.76) we have

$$
\begin{aligned}
\limsup _{T \rightarrow 0}\left[e^{\frac{\lambda}{6}\left(k-k_{i}(T)\right) T}\right] & \geq e^{\frac{\lambda}{6} \frac{T_{0}}{2}}, \quad k \in\left\{\bar{k}_{i}(T), \ldots, k_{i+1}(T)\right\}, i \in \mathbb{N} \\
& >1 .
\end{aligned}
$$

Hence, the claim is true. 
Using the result from Claim 1 in (5.74) yields

$$
\|\bar{\xi}[k]\| \leq e^{-\frac{\lambda}{3}\left(k-k_{i}(T)\right) T}\left\|\bar{\xi}\left[k_{i}(T)\right]\right\|+\frac{\gamma_{67}}{T^{n+1}}\|\bar{w}\|_{\infty}, \quad k \in\left\{\bar{k}_{i}(T), \ldots . k_{i+1}(T)\right\}, i \in \mathbb{N} .
$$

from which it immediately follows that

$$
\left\|\bar{\xi}\left[k_{i+1}(T)\right]\right\| \leq e^{-\frac{\lambda}{3}\left(k_{i+1}(T)-k_{i}(T)\right) T}\left\|\bar{\xi}\left[k_{i}(T)\right]\right\|+\frac{\gamma_{67}}{T^{n+1}}\|\bar{w}\|_{\infty}, \quad i \in \mathbb{N} .
$$

We now derive the bound for $\left\|\bar{\xi}\left[k_{i}(T)\right]\right\|$ in terms of $\|\bar{\xi}[0]\|$ and $\|\bar{w}\|_{\infty}$. Recalling that $k_{0}(T)=0$ by definition and $\gamma_{67} \geq \gamma_{56}$ by design, we have from Proposition 4 that

$$
\left\|\bar{\xi}\left[k_{1}(T)\right]\right\| \leq e^{-\frac{\lambda}{3} k_{1}(T) T}\|\bar{\xi}[0]\|+\frac{\gamma_{67}}{T^{n+1}}\|\bar{w}\|_{\infty} .
$$

Also, from (5.77):

$$
\begin{aligned}
\left\|\bar{\xi}\left[k_{2}(T)\right]\right\| & \leq e^{-\frac{\lambda}{3}\left(k_{2}(T)-k_{1}(T)\right) T}\left\|\bar{\xi}\left[k_{1}(T)\right]\right\|+\frac{\gamma_{67}}{T^{n+1}}\|\bar{w}\|_{\infty} \\
& \leq e^{-\frac{\lambda}{3}\left(k_{2}(T)-k_{1}(T)\right) T}\left[e^{-\frac{\lambda}{3} k_{1}(T) T}\|\bar{\xi}[0]\|+\frac{\gamma_{67}}{T^{n+1}}\|\bar{w}\|_{\infty}\right]+\frac{\gamma_{67}}{T^{n+1}}\|\bar{w}\|_{\infty} \\
& =e^{-\frac{\lambda}{3} k_{2}(T) T}\|\bar{\xi}[0]\|+\left[1+e^{-\frac{\lambda}{3}\left(k_{2}(T)-k_{1}(T)\right) T}\right] \frac{\gamma_{67}}{T^{n+1}}\|\bar{w}\|_{\infty} .
\end{aligned}
$$

It is easy to see that $\left(k_{i+1}(T)-k_{i}(T)\right) T>T_{0}-T$ for all $i \in \mathbb{N}$. Hence,

$$
\left\|\bar{\xi}\left[k_{2}(T)\right]\right\| \leq e^{-\frac{\lambda}{3} k_{2}(T) T}\|\bar{\xi}[0]\|+\left[1+e^{-\frac{\lambda}{3}\left(T_{0}-T\right)}\right] \frac{\gamma_{67}}{T^{n+1}}\|\bar{w}\|_{\infty} .
$$

Proceeding in this way we arrive at the sought after expression:

$$
\begin{aligned}
\left\|\bar{\xi}\left[k_{i}(T)\right]\right\| & \leq e^{-\frac{\lambda}{3} k_{i}(T) T}\|\bar{\xi}[0]\|+\left[1+e^{-\frac{\lambda}{3}\left(T_{0}-T\right)}+\cdots+e^{-\frac{\lambda}{3}(i-1)\left(T_{0}-T\right)}\right] \frac{\gamma_{67}}{T^{n+1}}\|\bar{w}\|_{\infty} \\
& \leq e^{-\frac{\lambda}{3} k_{i}(T) T}\|\bar{\xi}[0]\|+\frac{1-e^{-\frac{\lambda}{3} i\left(T_{0}-T\right)}}{1-e^{-\frac{\lambda}{3}\left(T_{0}-T\right)}} \frac{\gamma_{67}}{T^{n+1}}\|\bar{w}\|_{\infty} \\
& \leq e^{-\frac{\lambda}{3} k_{i}(T) T}\|\bar{\xi}[0]\|+\frac{1}{1-e^{-\frac{\lambda}{3}\left(T_{0}-T\right)}} \frac{\gamma_{67}}{T^{n+1}}\|\bar{w}\|_{\infty}, \quad i \in \mathbb{Z}^{+} .
\end{aligned}
$$

It is easy to see that there exists a constant $\gamma_{68}$ such that for small $T$ we have

$$
\left\|\bar{\xi}\left[k_{i}(T)\right]\right\| \leq e^{-\frac{\lambda}{3} k_{i}(T) T}\|\bar{\xi}[0]\|+\frac{\gamma_{68}}{T^{n+1}}\|\bar{w}\|_{\infty}, \quad i \in \mathbb{Z}^{+} .
$$

Substituting (5.78) into (5.74) we obtain for small $T$ :

$$
\begin{aligned}
\|\bar{\xi}[k]\| \leq & \left(1+\gamma_{65} T\right) e^{-\frac{\lambda}{2}\left(k-k_{i}(T)-1\right) T}\left[e^{-\frac{\lambda}{3} k_{i}(T) T}\|\bar{\xi}[0]\|+\frac{\gamma_{68}}{T^{n+1}}\|\bar{w}\|_{\infty}\right]+\frac{\gamma_{67}}{T^{n+1}}\|\bar{w}\|_{\infty} \\
\leq & \left(1+\gamma_{65} T\right) e^{\frac{\lambda}{2} T} e^{-\frac{\lambda}{2} k T} e^{\frac{\lambda}{6} k_{i}(T) T}\|\bar{\xi}[0]\|+\left[\left(1+\gamma_{65} T\right) e^{\frac{\lambda}{2} T} \gamma_{68}+\gamma_{67}\right] \frac{1}{T^{n+1}}\|\bar{w}\|_{\infty}, \\
& \quad k \in\left\{k_{i}(T), \ldots, k_{i+1}(T)\right\}, i \in \mathbb{Z}^{+} .
\end{aligned}
$$


Claim 2. For all $T \in(0, \tilde{T})$ it is true that

$$
e^{-\frac{\lambda}{2} k T} e^{\frac{\lambda}{6} k_{i}(T) T} \leq e^{-\frac{\lambda}{4} k T}, \quad k \in\left\{k_{i}(T), \ldots, k_{i+1}(T)\right\}, i \in \mathbb{Z}^{+} .
$$

Proof.

$$
\begin{aligned}
& & e^{-\frac{\lambda}{2} k T} e^{\frac{\lambda}{6} k_{i}(T) T} & \leq e^{-\frac{\lambda}{4} k T} \\
& \Longleftrightarrow & e^{\frac{\lambda}{6} k_{i}(T) T} & \leq e^{\frac{\lambda}{4} k T} \\
& & \frac{\lambda}{6} k_{i}(T) T & \leq \frac{\lambda}{4} k T \\
& \Longleftrightarrow & 3 k-2 k_{i}(T) & \geq 0
\end{aligned}
$$

which is true for all $k \in\left\{k_{i}(T), \ldots, k_{i+1}(T)\right\}, i \in \mathbb{Z}^{+}$.

Hence, there exist constants $\gamma_{69}$ and $\gamma_{70}$ such that for small $T$ we have

$$
\|\bar{\xi}[k]\| \leq \gamma_{69} e^{-\frac{\lambda}{4} k T}\|\bar{\xi}[0]\|+\frac{\gamma_{70}}{T^{n+1}}\|\bar{w}\|_{\infty}, \quad k \in \mathbb{Z}^{+}
$$

We now return to the original domain: the bound on $\|\xi[k]\|$ is derived as follows:

$$
\begin{aligned}
\|\xi[k]\| & \leq\left\|\left[\begin{array}{c}
X \\
Z
\end{array}\right]\right\|\|\bar{\xi}[k]\| \\
& \leq\left\|\left[\begin{array}{c}
X \\
Z
\end{array}\right]\right\|\left[\gamma_{69} e^{-\frac{\lambda}{4} k T}\|\bar{\xi}[0]\|+\frac{\gamma_{70}}{T^{n+1}}\|\bar{w}\|_{\infty}\right] \\
& \leq \underbrace{\left\|\left[\begin{array}{c}
X \\
Z
\end{array}\right]\right\|\left\|\left[\begin{array}{c}
X \\
Z
\end{array}\right]\right\|}_{\gamma_{16}} \gamma_{69} e^{-\frac{\lambda}{4} k T}\|\xi[0]\|+\underbrace{\left\|\left[\begin{array}{c}
X \\
Z
\end{array}\right]\right\| \gamma_{70}}_{=: \gamma_{71}} \frac{1}{T^{n+1}}\|\bar{w}\|_{\infty}, \quad k \in \mathbb{Z}^{+} .
\end{aligned}
$$

Therefore, at the sample points the response of the closed loop state to the initial condition is bounded by a decaying exponential plus a gain times the size of the noise.

We will now investigate the behaviour of the closed loop system state in between the sample points. To this end, using the bound (5.49) which holds even if $\alpha(t)$ is discontinuous, we have for $t \in[k T,(k+1) T)$ :

$$
\begin{aligned}
\left\|\left[\begin{array}{l}
x(t) \\
z(t)
\end{array}\right]\right\| & =\|x(t)\|+\|z(t)\| \\
& \leq \gamma_{36}\|x[k]\|+\gamma_{37} T\|z[k]\|+\frac{\gamma_{38}}{T^{n-2}}\|\bar{w}\|_{\infty}+\|z[k]\|, \quad k \in \mathbb{Z}^{+} .
\end{aligned}
$$


There exists a constant $\gamma_{72}$ so that for small $T$ we have

$$
\|\xi(t)\| \leq \gamma_{72}\|\xi[k]\|+\frac{\gamma_{38}}{T^{n-2}}\|\bar{w}\|_{\infty}, \quad t \in[k T,(k+1) T), k \in \mathbb{Z}^{+} .
$$

By combining (5.80) and (5.81), for small $T$ we obtain the following:

$$
\begin{gathered}
\|\xi(t)\| \leq \gamma_{72}\left[\gamma_{16} \gamma_{69} e^{-\frac{\lambda}{4} k T}\|\xi[0]\|+\frac{\gamma_{71}}{T^{n+1}}\|\bar{w}\|_{\infty}\right]+\frac{\gamma_{38}}{T^{n-2}}\|\bar{w}\|_{\infty} \\
\leq \gamma_{16} \gamma_{69} \gamma_{72} e^{-\frac{\lambda}{4} k T}\|\xi(0)\|+\left[\gamma_{71} \gamma_{72}+\gamma_{38} T^{3}\right] \frac{1}{T^{n+1}}\|\bar{w}\|_{\infty} \\
\quad t \in[k T,(k+1) T), k \in \mathbb{Z}^{+} .
\end{gathered}
$$

It is easy to see that for small $T$ there exists constants $\gamma_{73}$ and $\gamma_{74}$ such that

$$
\|\xi(t)\| \leq \gamma_{73} e^{-\frac{\lambda}{4} k T}\|\xi(0)\|+\frac{\gamma_{74}}{T^{n+1}}\|\bar{w}\|_{\infty}, \quad t \in[k T,(k+1) T), k \in \mathbb{Z}^{+} .
$$

Noting that $e^{-\frac{\lambda}{4} k T} \leq e^{-\frac{\lambda}{4}(t-T)}$ for $t \in[k T,(k+1) T)$, we can extend (5.82) to show that $\|\xi(t)\|$ is bounded for all $t \in \mathbb{R}^{+}$:

$$
\begin{aligned}
\|\xi(t)\| & \leq \gamma_{73} e^{-\frac{\lambda}{4}(t-T)}\|\xi(0)\|+\frac{\gamma_{74}}{T^{n+1}}\|\bar{w}\|_{\infty} \\
& \leq \gamma_{73} e^{\frac{\lambda}{4} T} e^{-\frac{\lambda}{4} t}\|\xi(0)\|+\frac{\gamma_{74}}{T^{n+1}}\|\bar{w}\|_{\infty} \\
& \leq \underbrace{\gamma_{73} e^{\frac{\lambda}{4}} \tilde{T}}_{=: \gamma_{75}} e^{-\frac{\lambda}{4} t}\|\xi(0)\|+\frac{\gamma_{74}}{T^{n+1}}\|\bar{w}\|_{\infty}, \quad t \in \mathbb{R}^{+} .
\end{aligned}
$$

It follows that the closed loop system is stable.

\subsection{Summary}

In this chapter we have built on the results derived in the noise free case. Here we considered the effect of noise and parameter discontinuities on the estimation process and the closed loop stability. Firstly, we derived a bound on the state estimation error:

$$
\|\hat{x}[k]-x[k]\| \leq \gamma_{x}\|x[k]\| h+\frac{\gamma_{20}}{h^{n-1}}\|\bar{w}\|_{\infty} .
$$

Here we see that both the size of the state and the size of the noise have an effect on the accuracy of the estimate. The effects are superpositioned due to the linearity of the plant 
and the estimator. We also see a clear trade-off where the fast sampling results in the greater effect of the noise on the accuracy of the estimate. Furthermore, in the absence of jumps, and when the combined plant-controller state is large enough, i.e.

$$
\left\|\left[\begin{array}{l}
x[k] \\
z[k]
\end{array}\right]\right\|>\frac{\gamma_{20}}{\left(1-\gamma_{x} h\right) h^{n}}\|\bar{w}\|_{\infty},
$$

we showed that the parameter estimation error is bounded by

$$
|\hat{\alpha}[k]-\alpha[k]| \leq \gamma_{26} h
$$

Having considered the behaviour of the closed loop system in the presence of persistent parameter jumps and the noise, we proved that the closed loop system is 5stable:

$$
\|\xi(t)\| \leq \gamma_{75} e^{-\frac{\lambda}{4} t}\|\xi(0)\|+\frac{\gamma_{74}}{T^{n+1}}\|\bar{w}\|_{\infty}, \quad t \in \mathbb{R}^{+} .
$$

In the above expression the response to the initial condition is bounded by a decaying exponential plus a gain times the size of the noise. The gain in front of the noise is potentially quite large, although it is uniformly bounded. 


\section{Chapter 6}

\section{Examples and Simulations}

\subsection{Controller Construction Algorithm}

In Section 3.2 it was noted that the design of our proposed controller is problematic for any general system because it is hard to satisfy Assumption 6. However, we can show that a solution can always be found for a certain class of second order time-varying systems.

Consider a second order time-varying system that satisfies Assumptions 1 through 5 as stated in Section 3.3 and has the form

$$
\begin{aligned}
& \dot{x}(t)=A(\alpha(t)) x(t)+B(\alpha(t)) u(t), \quad x(0)=x_{0} \in \mathbb{R}^{2} \\
& y(t)=C x(t) .
\end{aligned}
$$

We assume the system has the following properties:

- Property 1: The state matrix $A(\alpha(t))=\left[\begin{array}{ll}a_{11}(\alpha(t)) & a_{12}(\alpha(t)) \\ a_{21}(\alpha(t)) & a_{22}(\alpha(t))\end{array}\right], \quad \alpha \in \mathcal{A}$.

- Property 2: The uncertainty in $B(\alpha(t))$ enters only as a gain; without loss of generality we may assume that

$$
B(\alpha(t))=\left[\begin{array}{c}
\alpha(t) \\
0
\end{array}\right]=\alpha(t) \underbrace{\left[\begin{array}{l}
1 \\
0
\end{array}\right]}_{=: \bar{B}} .
$$


- Property 3: The matrix $C$ is constant.

Some additional constraints on the systems of this particular class are imposed by interpreting assumptions from Section 3.3.

- The controllability matrix is of the form $\alpha\left[\begin{array}{ll}1 & a_{11}(\alpha) \\ 0 & a_{21}(\alpha)\end{array}\right]$. Hence, to preserve controllability it must be that $0 \notin \mathcal{A}$ and $a_{21}(\alpha) \neq 0$ for all $\alpha \in \mathcal{A}$.

- Since $n=2$, we have that $\bar{n}=0$ by Assumption 4 ; this means that the parameter will have to be estimated from $C B(\alpha)=\alpha C \bar{B}$. Therefore, we require that $C \bar{B} \neq 0$.

- To satisfy Assumption 6, we will require that $a_{21}(\alpha)$ has the same sign for all $\alpha \in \mathcal{A}$. The reason for this will become clear soon.

By introducing $\bar{u}(t)=\alpha(t) u(t)$, the system (6.1)-(6.2) takes the form

$$
\begin{aligned}
\dot{x}(t) & =A(\alpha(t)) x(t)+\bar{B} \bar{u}(t), \quad x(0)=x_{0} \\
y(t) & =C x(t) .
\end{aligned}
$$

Note that if we can find matrices $X, P(\alpha)$ and $\bar{U}(\alpha)$ satisfying $A(\alpha) X+\bar{B} \bar{U}(\alpha)=X P(\alpha)$, then by setting $U(\alpha)=\bar{U}(\alpha) / \alpha$, we have that $A(\alpha) X+B(\alpha) U(\alpha)=X P(\alpha)$ is also satisfied.

Theorem 3. For any system of form (6.3)-(6.4) it is possible to find a solution to

$$
A(\alpha) X+\bar{B} \bar{U}(\alpha)=X P(\alpha)
$$

where $X$ is a $2 \times 2$ full-rank matrix and $P(\alpha) \in \mathcal{H}_{1}$ is absolutely continuous and has a bounded derivative on $\mathcal{A}$.

Proof. The analysis that follows might be hard to understand without providing extensive background on invariant set theory, which is outside the scope of this work. However, 
we will provide some intuition with respect to constructing matrices $X, U(\alpha)$ and $P(\alpha)$ and prove that they indeed satisfy equation (6.5). We will use the geometric approach as explained in [6]. The goal is to find a convex, balanced, and absorbing set $\mathcal{S} \subset \mathbb{R}^{2}$ so that for every $x_{0} \in \mathcal{S}$, the controller maintains $x(t) \in \mathcal{S}$ and $x(t) \rightarrow 0$ as $t \rightarrow \infty$. If we do an Euler approximation of (6.3), we need, for every $x \in \mathcal{S}$ and $\alpha \in \mathcal{A}$ and for some $T>0$, that there exist a control signal $\bar{u} \in \mathbb{R}$ satisfying

$$
x+T[A(\alpha) x+\bar{B} \bar{u}] \in \mathcal{S} .
$$

Let $\mathcal{S}$ be the polyhedral set whose vertices are defined by the columns of $[X-X]$. If we let $X=\left[\begin{array}{ll}\bar{x}_{1} & \bar{x}_{2}\end{array}\right]$, then $\bar{U}(\alpha)=\left[\bar{u}_{1}(\alpha) \bar{u}_{2}(\alpha)\right]$ is such that the vertices of $\mathcal{S}$ are mapped to its interior, i.e. for some $T>0$

$$
\bar{x}_{j}+T\left[A(\alpha) \bar{x}_{j}+\bar{B} \bar{u}_{j}(\alpha)\right] \in \operatorname{int}\{\mathcal{S}\} \quad \text { for } j=1,2 \text { and all } \alpha \in \mathcal{A} .
$$

Of course, only one half of the vertices must be considered due to symmetry. Without loss of generality we will consider matrix $X$ where

$$
\bar{x}_{1}=\left[\begin{array}{l}
\bar{x} \\
1
\end{array}\right] \text { and } \bar{x}_{2}=\left[\begin{array}{c}
-\bar{x} \\
0
\end{array}\right] \text { for some } \bar{x} \in \mathbb{R} .
$$

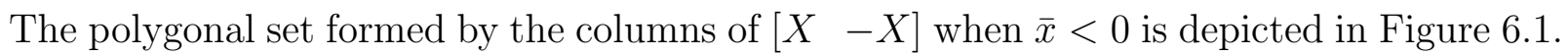
Condition (6.6) is satisfied when the vectors $A(\alpha) \bar{x}_{1}+\bar{B} \bar{u}_{1}(\alpha)$ and $A(\alpha) \bar{x}_{2}+\bar{B} \bar{u}_{2}(\alpha)$ point inside the polygon for all $\alpha \in \mathcal{A}$. Note that vector $\bar{B} \bar{u}_{j}(\alpha)$ is always directed strictly horizontally. With that in mind, consider vertex $\bar{x}_{1}$, which is located in the upper half of the plane: it is imperative that $A(\alpha) \bar{x}_{1}$ has a downward vertical component, i.e

$$
a_{21}(\alpha) \bar{x}+a_{22}(\alpha)<0 \quad \text { for all } \alpha \in \mathcal{A} \text {. }
$$

Since $a_{21}(\alpha)$ has the same sign for all $\alpha \in \mathcal{A}$, we can always choose $\bar{x}$ such that

$$
a_{21}(\alpha) \bar{x}<0 \quad \text { for all } \quad \alpha \in \mathcal{A}
$$

Also, because the set $\mathcal{A}$ is compact, there exist constants

$$
\underline{a}_{21}=\min _{\alpha \in \mathcal{A}} a_{21}(\alpha), \quad \bar{a}_{21}=\max _{\alpha \in \mathcal{A}} a_{21}(\alpha), \quad \text { and } \quad \bar{a}_{22}=\max _{\alpha \in \mathcal{A}} a_{22}(\alpha) .
$$

Suppose $a_{21}(\alpha)$ is always positive; then we choose $\bar{x}$ to be negative and (6.7) will be satisfied if the following is true:

$$
\underline{a}_{21} \bar{x}+\bar{a}_{22}<0 \text {. }
$$




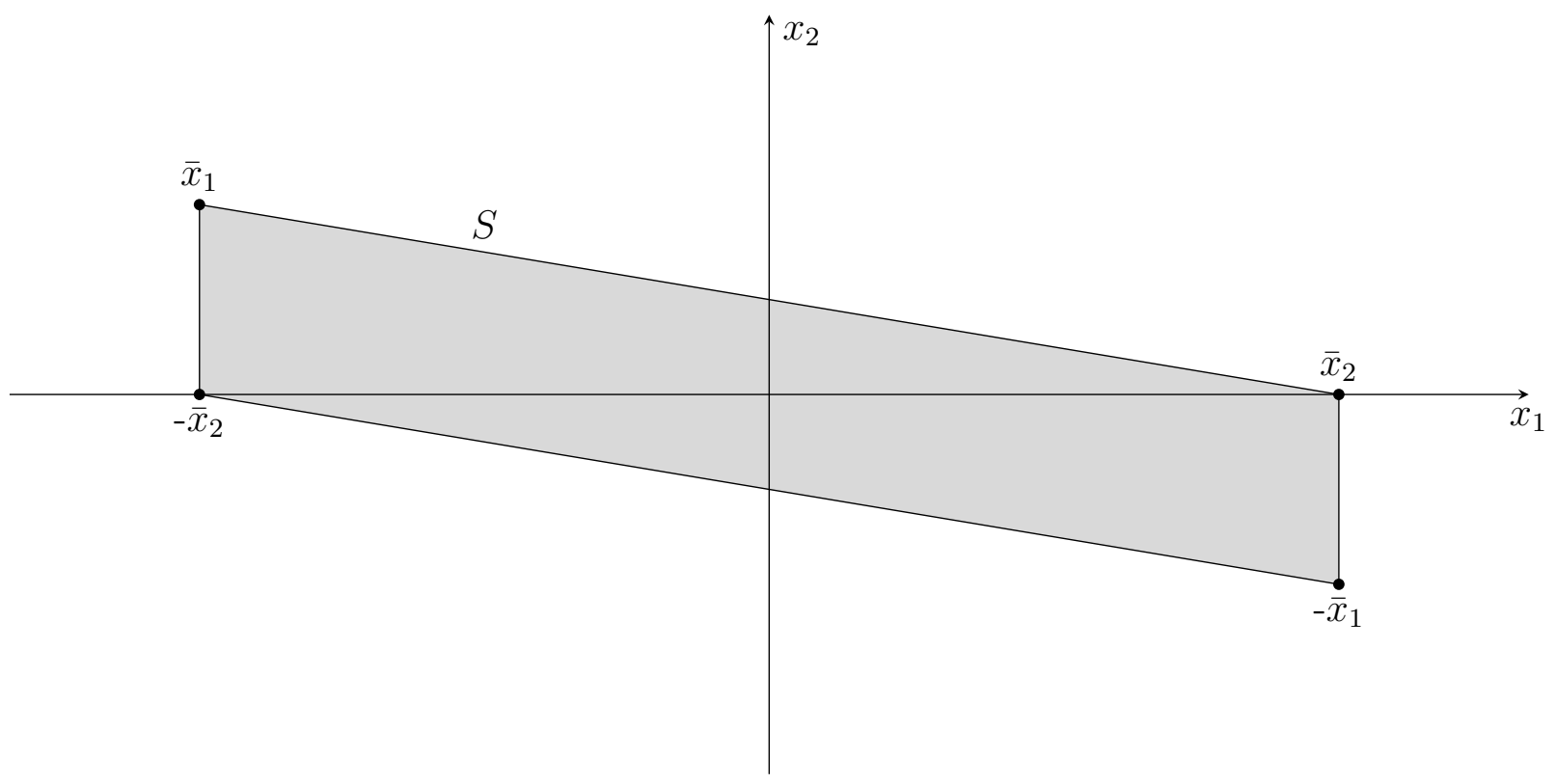

Figure 6.1: Polygonal set.

The above yields the constraint

$$
\bar{x}<-\frac{\bar{a}_{22}}{\underline{a}_{21}},
$$

from which we can choose a conservative value for $\bar{x}$ :

$$
\bar{x}=-\left|\frac{\bar{a}_{22}}{\underline{a}_{21}}\right|-1 .
$$

Having ascertained that a solution can be found, we move on to calculate an appropriate $\bar{u}_{1}(\alpha)$. Let us first examine $A(\alpha) \bar{x}_{1}+\bar{B} \bar{u}_{1}(\alpha)$ :

$$
A(\alpha) \bar{x}_{1}+\bar{B} u_{1}(\alpha)=\left[\begin{array}{c}
a_{11}(\alpha) \bar{x}+a_{12}(\alpha)+u_{1}(\alpha) \\
a_{21}(\alpha) \bar{x}+a_{22}(\alpha)
\end{array}\right]
$$

Referring to Figure 6.1, we would like the slope of $A(\alpha) \bar{x}_{1}+\bar{B} \bar{u}_{1}(\alpha)$ to be more negative than the slope of the line connecting $\bar{x}_{1}$ and $\bar{x}_{2}$, which is equal to $1 /(2 \bar{x})$. Hence, we can conservatively choose the slope to be $1 / \bar{x}$, which yields the control signal

$$
\bar{u}_{1}(\alpha)=-a_{11}(\alpha) \bar{x}-a_{12}(\alpha)+\bar{x}\left[a_{21}(\alpha) \bar{x}+a_{22}(\alpha)\right] .
$$


Now consider $A(\alpha) \bar{x}_{2}+\bar{B} u_{2}(\alpha)$ :

$$
A(\alpha) \bar{x}_{2}+\bar{B} u_{2}(\alpha)=\left[\begin{array}{c}
-a_{11}(\alpha) \bar{x}+u_{2}(\alpha) \\
-a_{21}(\alpha) \bar{x}
\end{array}\right]
$$

where $-a_{21}(\alpha) \bar{x}$ is always positive. We need to choose $\bar{u}_{2}(\alpha)$ such that the slope of $A(\alpha) \bar{x}_{2}+$ $\bar{B} \bar{u}_{2}(\alpha)$ is between 0 and $1 /(2 \bar{x})$. We pick $1 /(4 \bar{x})$ as a convenient choice and obtain

$$
\bar{u}_{2}(\alpha)=a_{11}(\alpha) \bar{x}-4 a_{21}(\alpha) \bar{x}^{2} .
$$

Following in the same steps, it is easy to check that when $a_{21}(\alpha)<0$, we choose $\bar{x}$ to be positive, specifically

$$
\bar{x}=\left|\frac{\bar{a}_{22}}{\bar{a}_{21}}\right|+1
$$

The resulting polygon is basically a mirror reflection of the one shown in Figure 6.1. Intuitively, since the signs of $\bar{x}$ and $a_{21}(\alpha)$ have changed, the expressions for $\bar{u}_{1}(\alpha)$ and $\bar{u}_{2}(\alpha)$ remain unchanged from (6.10) and (6.11) respectively. The matrix $P(\alpha)$ is easily calculated because $X$ is square and invertible by construction:

$$
P(\alpha)=X^{-1}[A(\alpha) X+\bar{B} \bar{U}(\alpha)] .
$$

The only thing left to show is that $P(\alpha)$ belongs to $\mathcal{H}_{1}$. Substituting the expressions for $A(\alpha), X, \bar{B}$, and $\bar{U}(\alpha)$ into (6.13), and noting that the inverse of $X$ is simply

$$
X^{-1}=\left[\begin{array}{rr}
0 & 1 \\
-\frac{1}{\bar{x}} & 1
\end{array}\right]
$$

we have

$$
P(\alpha)=\left[\begin{array}{cc}
a_{21}(\alpha) \bar{x}+a_{22}(\alpha) & -a_{21}(\alpha) \bar{x} \\
0 & 3 a_{21}(\alpha) \bar{x}
\end{array}\right] .
$$

Consequently,

$$
\|I+T P(\alpha)\|=\max \left\{\left|1+T\left[a_{21}(\alpha) \bar{x}+a_{22}(\alpha)\right]\right|,\left|1+3 T a_{21} \bar{x}\right|+T\left|a_{21} \bar{x}\right|\right\} .
$$

Since both $a_{21}(\alpha) \bar{x}+a_{22}(\alpha)$ and $a_{21} \bar{x}$ are always negative by construction, it is clear that for small $T$ we have $\|I+T P(\alpha)\|<1$ for all $\alpha \in \mathcal{A}$.

As a consequence of Theorem 3 and Remark 2, the stabilizing control signal for (6.1)(6.2) can be calculated as follows:

$$
u_{s}[k]=U(\hat{\alpha}[k]) X^{-1} \hat{x}[k]=\frac{1}{\hat{\alpha}[k]} \bar{U}(\hat{\alpha}[k]) X^{-1} \hat{x}[k] .
$$




\subsection{Simulations}

Example 1. Consider the following system:

$$
\begin{aligned}
\dot{x}(t) & =\left[\begin{array}{cc}
0 & 1 \\
1 & 1+4 \alpha(t)
\end{array}\right] x(t)+\alpha(t)\left[\begin{array}{l}
1 \\
0
\end{array}\right] u(t), \quad x(0)=\left[\begin{array}{c}
10 \\
5
\end{array}\right] \\
y(t) & =\left[\begin{array}{ll}
1 & 0
\end{array}\right] x(t) .
\end{aligned}
$$

The parameter takes values in the set $\mathcal{A}=[-2,-1] \cup[1,2]$ with a bound on the derivative $|\dot{\alpha}(t)| \leq 10$ and the minimum time between jumps $T_{0}=0.5$ seconds.

The transfer function of the plant is

$$
\alpha \frac{s-4 \alpha-1}{s^{2}-(4 \alpha+1) s-1},
$$

from which it is easy to see that the plant is unstable for all $\alpha \in \mathcal{A}$ and has a zero in the open right half plane when $\alpha \in[1,2]$.

The plant clearly fits the paradigm of Section 6.1, and therefore, we can construct the controller as per the suggested algorithm. The parameter can be easily estimated from $f(\alpha)=\alpha C B=\alpha$. The controller matrices are

$$
X=\left[\begin{array}{cc}
-10 & 10 \\
1 & 0
\end{array}\right] \quad \text { and } \quad U(\hat{\alpha}[k])=\frac{1}{\hat{\alpha}[k]}[-1-10(-9+4 \hat{\alpha}[k]) \quad-400]
$$

which yield the stabilizing control signal

$$
u_{s}[k]=U(\hat{\alpha}[k]) X^{-1} \hat{x}[k] .
$$

For simulations we chose $h=0.001$ seconds, $T_{1}=2 h, T_{2}=2 h, T_{3}=6 h$ (yielding $T=0.01 \mathrm{~s}$ ) and $\rho=1$. The parameter was modeled as switching between two trajectories:

$$
\alpha(t)=1+\cos ^{2}(10 t) \text { and } \alpha(t)=-1-\cos ^{2}(10 t)
$$

with no more than two jumps per second. We modeled noise as a $60 \mathrm{~Hz}$ sinusoidal waveform:

$$
\begin{aligned}
d(t) & =10^{-3} \cos (120 \pi t), \\
w(t) & =10^{-3} \cos (120 \pi t) .
\end{aligned}
$$

The simulation results are presented in Figures 6.2 and 6.3. We can see that the output signal is bounded in response to the initial condition and the noise. The control signal 

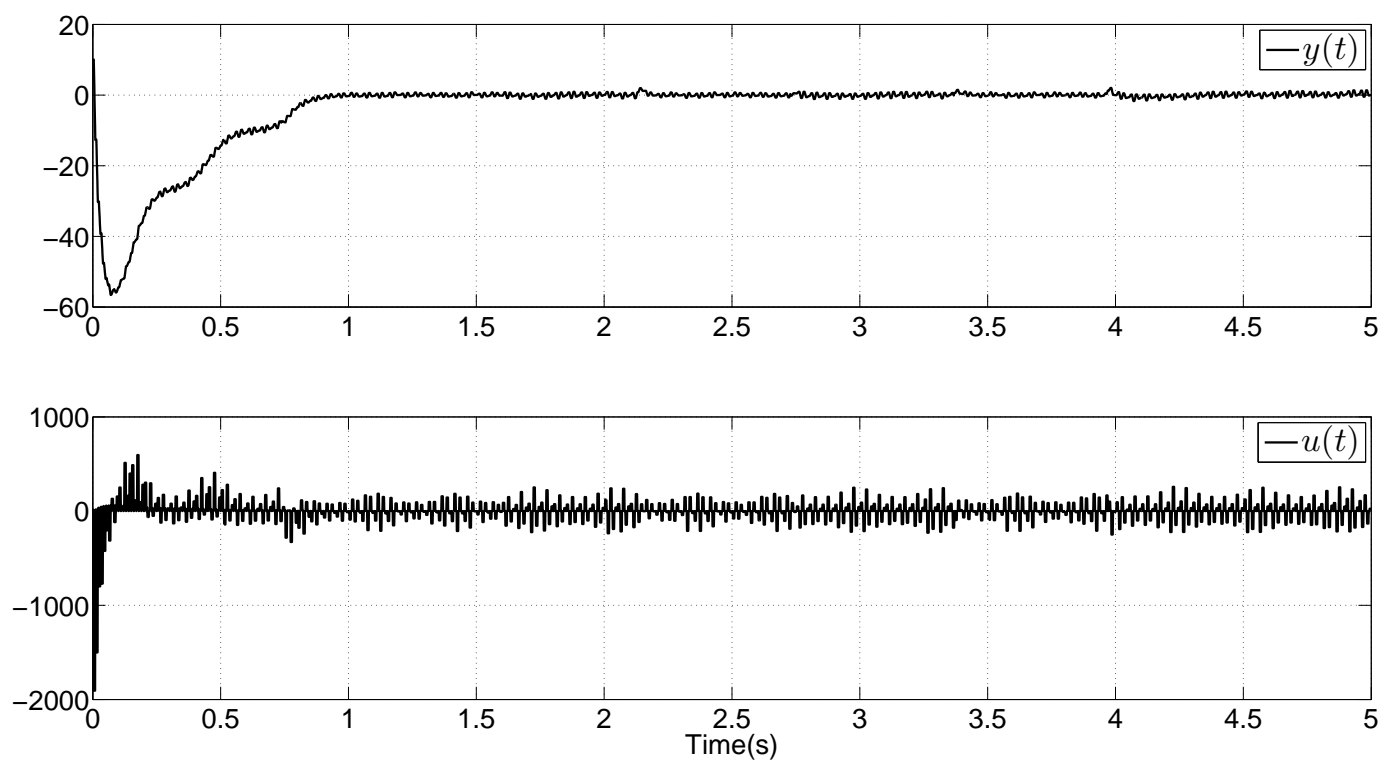

Figure 6.2: Example 1: output and control signals.
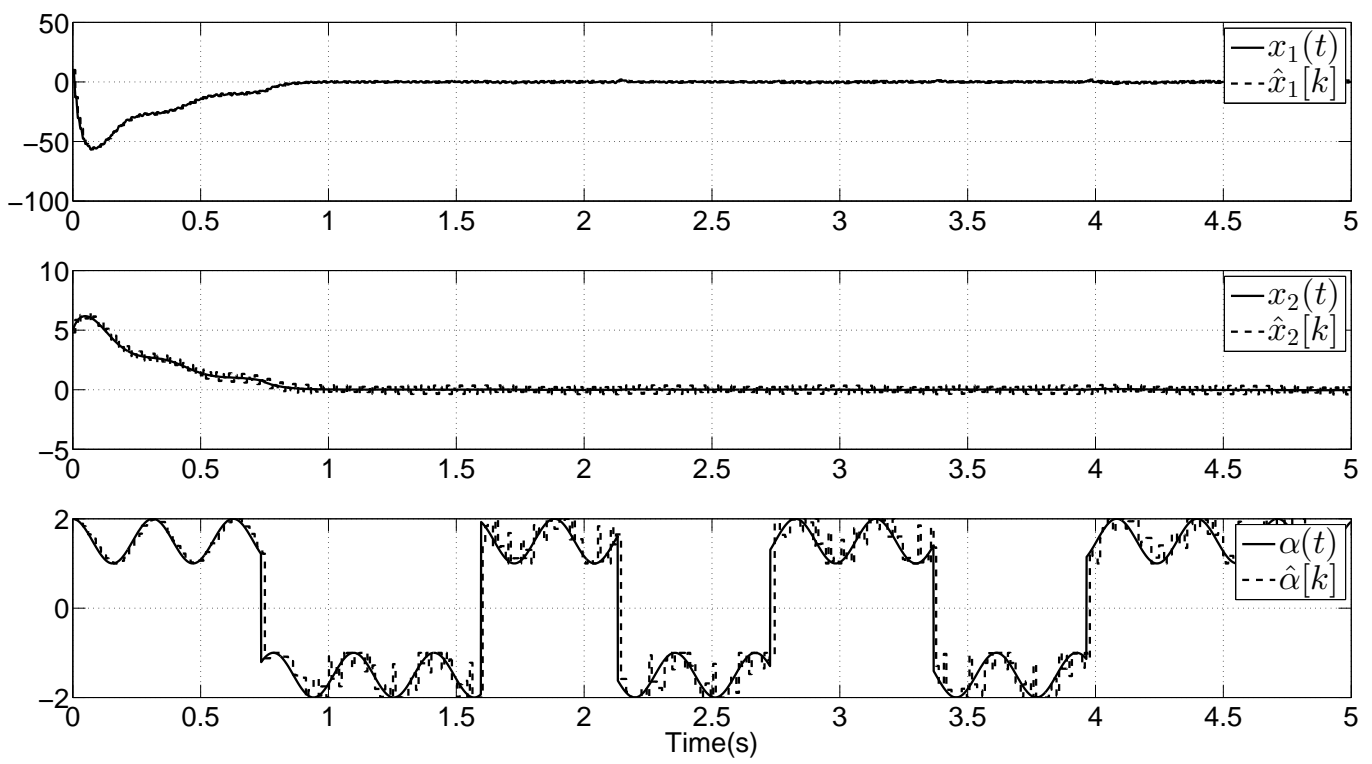

Figure 6.3: Example 1: trajectories of the states and the parameter. 
reaches large values in the beginning, but becomes moderate later. However, fast actuators will be required if the controller is to be implemented on a physical system. Also, in Figure 6.3 we observe that although the parameter estimates become poor when the state decreases in size, the estimate is always in the correct interval.

Example 2. Now we consider a system which has a varying matrix $B$ :

$$
\begin{aligned}
& \dot{x}(t)=\left[\begin{array}{cc}
-1 & 1 \\
10-20 \alpha & 1-8(\alpha-0.5)^{2}
\end{array}\right] x(t)+\left[\begin{array}{c}
1-2 \alpha \\
1-4(\alpha-0.5)^{2}
\end{array}\right] u(t), \quad x(0)=\left[\begin{array}{c}
10 \\
5
\end{array}\right] \\
& y(t)=\left[\begin{array}{ll}
1 & 0
\end{array}\right] x(t),
\end{aligned}
$$

where $\alpha(t)$ takes values in $\mathcal{A}=[0,1]$, with the rate of change $|\dot{\alpha}(t)| \leq 4$, and the minimum time between jumps $T_{0}=0.25$ seconds.

The transfer function of the plant is

$$
\frac{(1-2 \alpha) s-\left(16 \alpha^{3}-20 \alpha^{2}+6 \alpha\right)}{s^{2}+\left(8 \alpha^{2}-8 \alpha+2\right) s+\left(8 \alpha^{2}+12 \alpha-9\right)} .
$$

It is not hard to verify that the system is unstable for all $\alpha \in[0,0.549]$ and has a zero in the open right half plane for $\alpha \in(0,0.5) \cup(0.5,0.75)$.

This example is quite interesting because in contrast to Example 1, here $B(\alpha)$ changes direction depending on the value of the parameter. In fact, it goes through a full $180^{\circ}$ rotation as $\alpha$ varies from 0 to 1 . This made satisfying Assumption 6 more challenging, especially since the algorithm developed in Section 6.1 could not be applied here. We had to resort to ad-hoc techniques and a solution was found with

$$
X=\left[\begin{array}{rc}
-0.9603 & 0.0358 \\
0.2790 & 0.3783
\end{array}\right] \text { and } U(\alpha)=\left[u_{1}(\alpha), u_{2}(\alpha)\right]
$$

where $u_{1}(\alpha)$ and $u_{2}(\alpha)$ respectively were:

$$
\begin{gathered}
u_{1}(\alpha)=\left\{\begin{array}{cl}
-26 \alpha+13 & \alpha \in[0,0.25) \\
-20(\alpha-0.2)+5 & \alpha \in[0.25,0.75) \\
-10-340(\alpha-0.75) & \alpha \in[0.75,1]
\end{array}\right. \\
u_{2}(\alpha)=\left\{\begin{array}{cl}
-0.5-1.5 \alpha & \alpha \in[0,0.25) \\
-10 & \alpha \in[0.25,0.75) \\
-20(0.9-\alpha) & \alpha \in[0.75,1] .
\end{array}\right.
\end{gathered}
$$

Note however, that $u_{1}(\alpha)$ and $u_{2}(\alpha)$ are discontinuous at $\alpha=0.25$ and $\alpha=0.75$ as can 

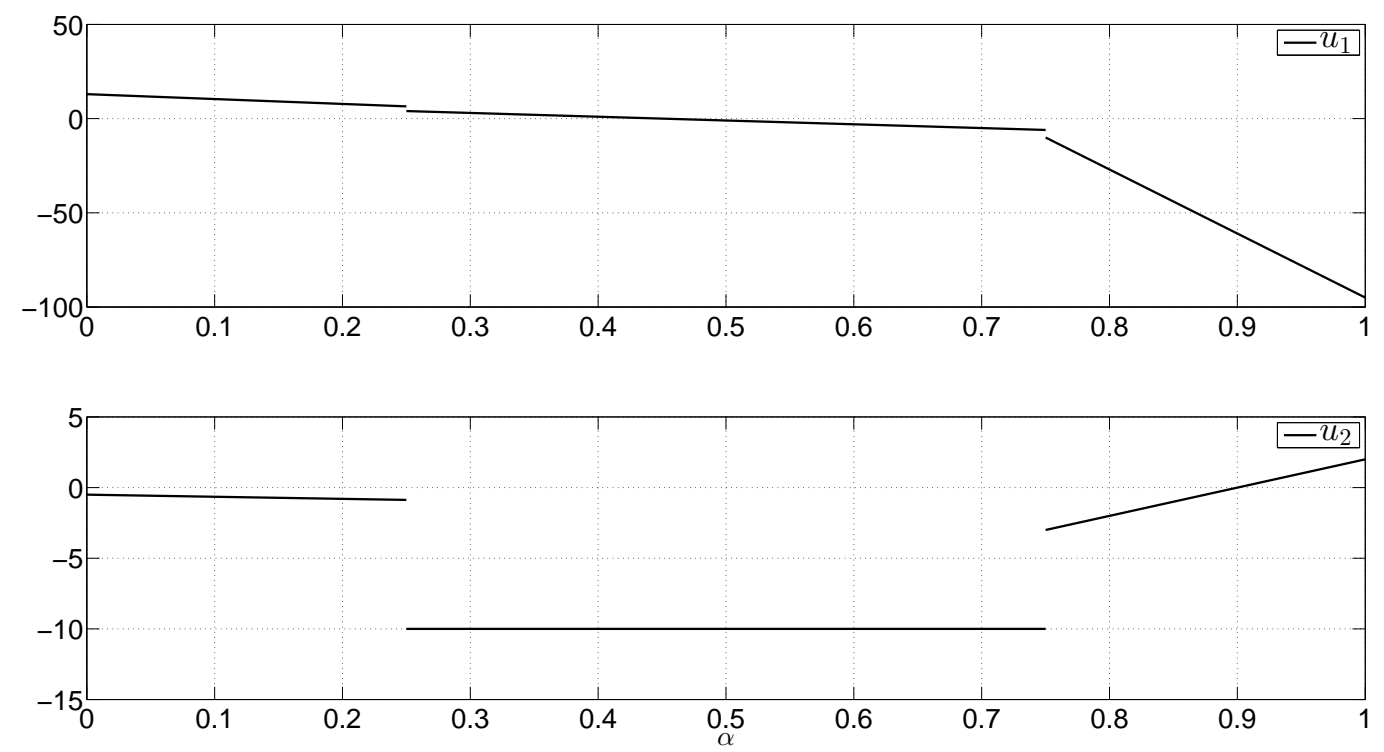

Figure 6.4: Example 2: discontinuous $u_{1}$ and $u_{2}$.
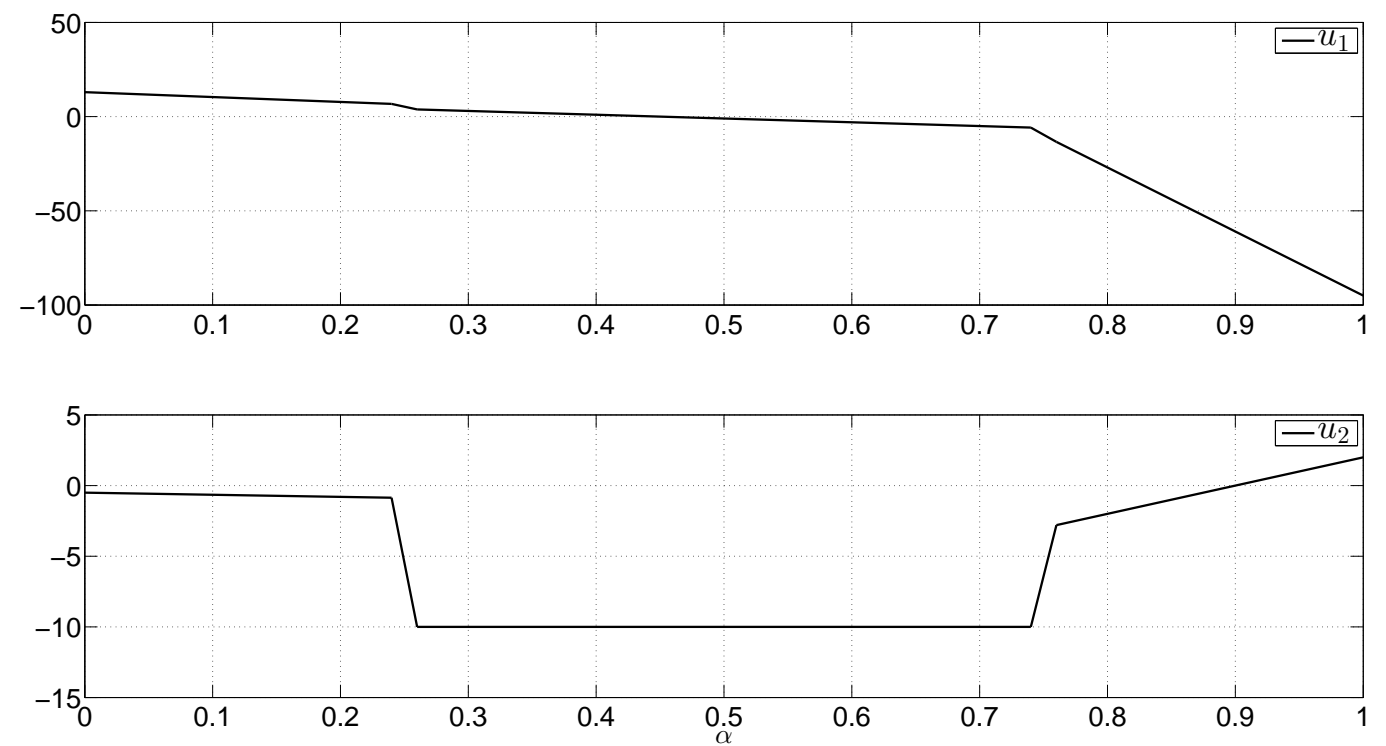

Figure 6.5: Example 2: Piecewise continuous $u_{1}$ and $u_{2}$ after interpolation. 
be seen from Figure 6.4. This violates our assumption that $U(\alpha)$ is continuous. Hence, it was required to transform $u_{1}(\alpha)$ and $u_{2}(\alpha)$ into piecewise continuous signals, which was achieved by interpolating around the points of discontinuity as displayed in Figure 6.5. This yielded a piecewise continuous $U(\hat{\alpha}[k])$ and, since $X$ is square, the stabilizing control law was calculated simply as

$$
u_{s}[k]=U(\hat{\alpha}[k]) X^{-1} \hat{x}[k]
$$

Note that $f(\alpha)=C B(\alpha)=1-2 \alpha$, which allowed for straightforward parameter estimation.

For simulations we once again chose $h=0.001$ seconds, $T_{1}=2 h, T_{2}=2 h, T_{3}=6 h$ and $\rho=1$. The parameter trajectory was chosen to be a sawtooth signal that varied from 0 to 1 with a period of 0.25 seconds. Sinusoidal noise was added at the input and output interfaces:

$$
\begin{aligned}
d(t) & =10^{-3} \cos (120 \pi t), \\
w(t) & =10^{-3} \cos (120 \pi t) .
\end{aligned}
$$

The results of the simulation are shown in Figures 6.6 and 6.7. The plant is stabilized very quickly, although the control signal is quite large in the beginning and switches rapidly. As expected, the parameter estimate becomes poor when the plant states are close to zero, although this has little effect on the response. 

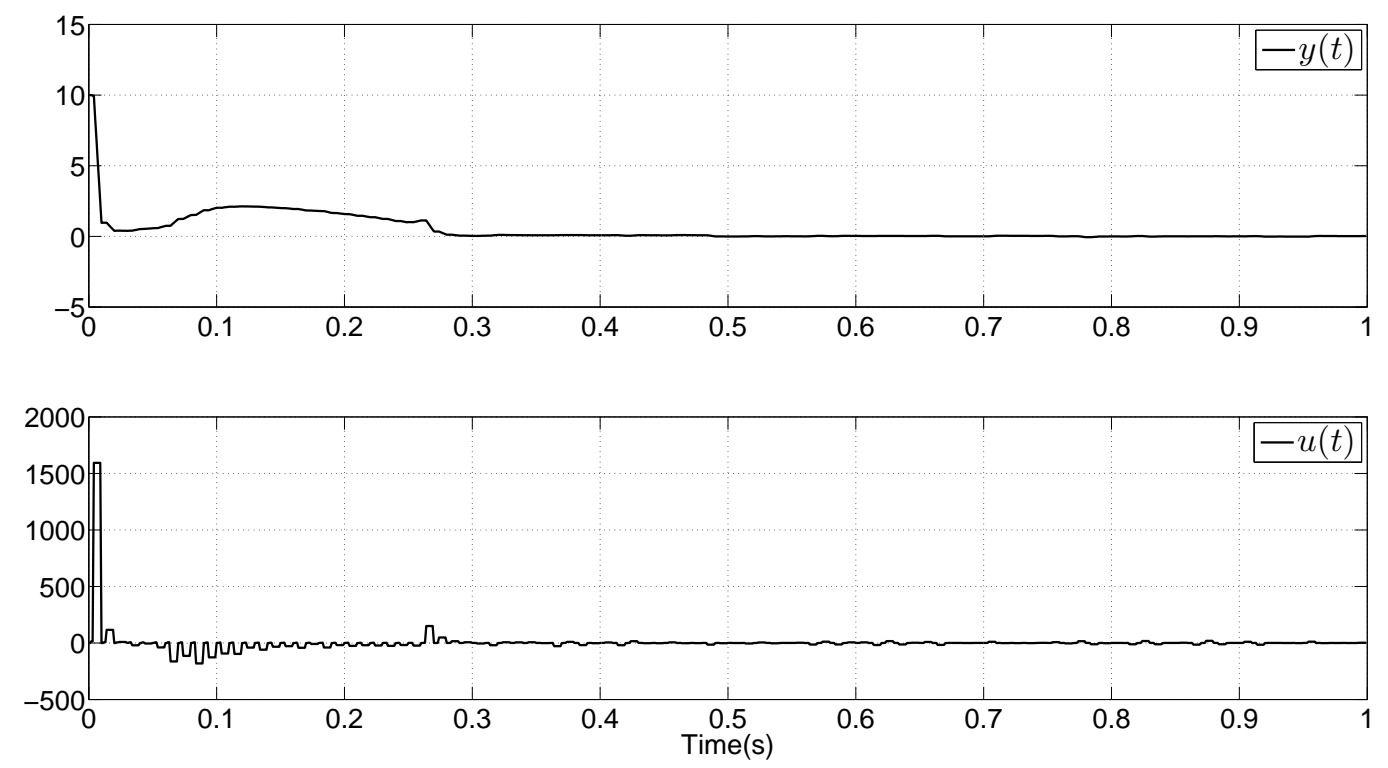

Figure 6.6: Example 2: output and control signals.
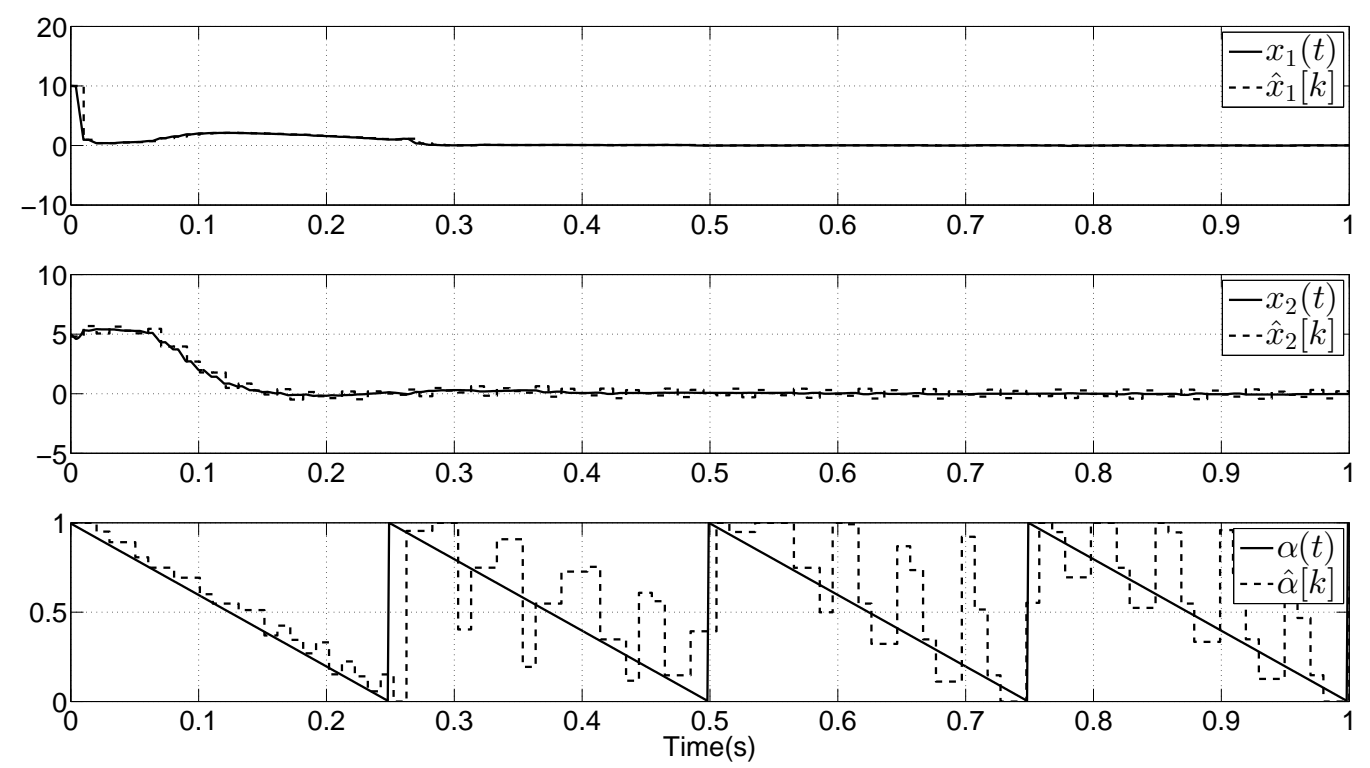

Figure 6.7: Example 2: trajectories of the states and the parameter. 


\section{Chapter 7}

\section{Conclusions}

In this thesis we considered a problem of adaptively stabilizing rapidly time-varying linear systems. While there are solutions where the plant is assumed to have stable zero dynamics, there are no general results for rapidly time-varying systems with unstable zero dynamics. Here we have looked at a class of such systems and constructed a nonlinear adaptive controller that provides stability.

This work was largely motivated by a result in the area of gain scheduling [5], where it was shown that, assuming the parameter values are measurable, a stabilizing controller can be designed to handle fast parameter variations. We have adopted the gain scheduling approach to calculate the stabilizing control signal; however, we made the controller adaptive by adding a state estimator and an estimator of the free parameter. We have assumed that the plant is parametrized by a scalar parameter that belongs to a compact set. Since the controller we use is sampled-data, we impose a bound on the rate of change of the parameter; however, we do allow for occasional jumps. We have proved a superposition-like bound on the effects of initial condition and the noise on the state: the response to the initial condition is bounded by a decaying exponential plus a gain times the size of the noise.

As it was pointed out in the beginning of this work, there are many assumptions that the plant must satisfy for our procedure to be applied. Despite this, we have identified one class of interesting examples for which the compensator design is relatively straightforward. There may exist more classes of systems where our procedure is applicable; however, due to the complexity of conditions in [5], they are hard to pinpoint. Therefore, it would be beneficial if in future work some effort was dedicated to researching ways of solving the aforementioned conditions or developing simpler procedures of checking them. In 
particular, it could be possible to replace Assumption 3 with a possibly less restrictive assumption that the system satisfies the observability condition in [5]. In such hypothetical scenario, the analysis of the closed loop stability does not follow in a straightforward manner from the work presented herein; hence, it is difficult to comment on the restrictiveness of the new assumption. 
APPENDICES 


\section{Appendix A}

\section{Proofs}

Proof of Proposition 1. Consider an $m \times m$ matrix $H(\alpha)$, which lies in $\mathcal{H}_{1}$ :

$$
H(\alpha)=\left[\begin{array}{cccc}
h_{11}(\alpha) & h_{12}(\alpha) & \cdots & h_{1 m}(\alpha) \\
h_{21}(\alpha) & h_{22}(\alpha) & \cdots & h_{2 m}(\alpha) \\
\vdots & \vdots & \ddots & \vdots \\
h_{m 1}(\alpha) & h_{m 2}(\alpha) & \cdots & h_{m m}(\alpha)
\end{array}\right]
$$

Recall that by definition of $\mathcal{H}_{1}$, for $T \in(0, \bar{\tau})$ we have

$$
\|I+T H(\alpha)\|<1, \quad \alpha \in \mathcal{A} .
$$

For simplicity suppose that for now $\alpha \in \mathcal{A}$ is fixed. Let us examine $I+T H(\alpha)$ :

$$
I+T H(\alpha)=\left[\begin{array}{cccc}
1+T h_{11}(\alpha) & T h_{12}(\alpha) & \ldots & T h_{1 m}(\alpha) \\
T h_{21}(\alpha) & 1+T h_{22}(\alpha) & \ldots & T h_{2 m}(\alpha) \\
\vdots & \vdots & \ddots & \vdots \\
T h_{m 1}(\alpha) & T h_{m 2}(\alpha) & \ldots & 1+T h_{m m}(\alpha)
\end{array}\right]
$$

Note that (A.1) is equivalent to

$$
\max _{j}\left(\left|1+T h_{j j}(\alpha)\right|+T \sum_{\substack{i=1 \\ i \neq j}}^{m}\left|h_{i j}(\alpha)\right|\right)<1
$$


which, in turn, translates to

$$
\left|1+T h_{j j}(\alpha)\right|+T \sum_{\substack{i=1 \\ i \neq j}}^{m}\left|h_{i j}(\alpha)\right|<1 \text { for all } j=1,2, \ldots, m .
$$

The above means that, in particular, $\left|1+T h_{j j}(\alpha)\right|<1$ must hold for all $j=1,2, \ldots, m$. This is true for $T \in(0, \bar{\tau})$ if and only if $h_{j j}(\alpha)<0$ for all $j=1,2, \ldots, m$. We would like to choose $T^{*} \in(0, \bar{\tau})$ such that $1+T h_{j j}(\alpha)>0$ for all $T \in\left(0, T^{*}\right)$ and $j=1,2, \ldots, m$. If we define

$$
T^{*}:=\min \left\{\frac{1}{\max _{j}\left|h_{j j}(\alpha)\right|}, \frac{\bar{\tau}}{2}\right\},
$$

then for all $T \in\left(0, T^{*}\right)$ we have that $1+T h_{j j}(\alpha)$ is positive and equation (A.2) transforms to

$$
h_{j j}(\alpha)+\sum_{\substack{i=1 \\ i \neq j}}^{m}\left|h_{i j}(\alpha)\right|<0 \quad \text { for all } j=1,2, \ldots, m .
$$

We will now prove a result that is stronger than (A.1), i.e. there exists $\lambda^{*}>0$ such that for all $\lambda \in\left(0, \lambda^{*}\right)$ and $T \in\left(0, T^{*}\right)$ we have

$$
\|I+T H(\alpha)\|<1-\lambda T,
$$

which is equivalent to

$$
h_{j j}(\alpha)+\sum_{\substack{i=1 \\ i \neq j}}^{m}\left|h_{i j}(\alpha)\right|<-\lambda \text { for all } j=1,2, \ldots, m .
$$

Having already established that the left hand side is negative, in order for (A.5) to hold we need

$$
\lambda<\left|h_{j j}(\alpha)+\sum_{\substack{i=1 \\ i \neq j}}^{m}\right| h_{i j}(\alpha)|| \text { for all } j=1,2, \ldots, m .
$$

Defining $\lambda^{*}$ as

$$
\lambda^{*}:=\min _{j}\left|h_{j j}(\alpha)+\sum_{\substack{i=1 \\ i \neq j}}^{m}\right| h_{i j}(\alpha)||,
$$

which is greater than zero because of (A.3), we see that (A.4) is clearly satisfied for all $\lambda \in\left(0, \lambda^{*}\right)$ and $T \in\left(0, T^{*}\right)$. 
Of course, the parameter $\alpha$ is not fixed, since it is free to vary among the compact set $\mathcal{A}$, and (3.8) must be satisfied for all $\alpha \in \mathcal{A}$. To this end, we define

$$
\bar{T}:=\min \left\{\frac{1}{\max _{\alpha} \max _{j}\left|h_{j j}(\alpha)\right|}, \frac{\bar{\tau}}{2}\right\}=\min \left\{\frac{1}{\max _{j} \max _{\alpha}\left|h_{j j}(\alpha)\right|}, \frac{\bar{\tau}}{2}\right\}
$$

and

$$
\bar{\lambda}:=\min _{\alpha} \min _{j}\left|h_{j j}(\alpha)+\sum_{\substack{i=1 \\ i \neq j}}^{m}\right| h_{i j}(\alpha)||=\min _{j} \min _{\alpha}\left|h_{j j}(\alpha)+\sum_{\substack{i=1 \\ i \neq j}}^{m}\right| h_{i j}(\alpha)|| .
$$

It is easy to see that (3.8) is satisfied for all $T \in(0, \bar{T})$ and $\lambda \in(0, \bar{\lambda})$.

Proof of Proposition 2. Consider an $m \times m$ matrix $H(\alpha)$, which lies in $\mathcal{H}_{\infty}$ :

$$
H(\alpha)=\left[\begin{array}{cccc}
h_{11}(\alpha) & h_{12}(\alpha) & \cdots & h_{1 m}(\alpha) \\
h_{21}(\alpha) & h_{22}(\alpha) & \cdots & h_{2 m}(\alpha) \\
\vdots & \vdots & \ddots & \vdots \\
h_{m 1}(\alpha) & h_{m 2}(\alpha) & \cdots & h_{m m}(\alpha)
\end{array}\right]
$$

Remember that by definition of $\mathcal{H}_{\infty}$, for $T \in(0, \bar{\tau})$ we have

$$
\|I+T H(\alpha)\|_{\infty}<1, \quad \alpha \in \mathcal{A} .
$$

For simplicity suppose that for now $\alpha \in \mathcal{A}$ is fixed. Let us examine $I+T H(\alpha)$ :

$$
I+T H(\alpha)=\left[\begin{array}{cccc}
1+T h_{11}(\alpha) & T h_{12}(\alpha) & \ldots & T h_{1 m}(\alpha) \\
T h_{21}(\alpha) & 1+T h_{22}(\alpha) & \cdots & T h_{2 m}(\alpha) \\
\vdots & \vdots & \ddots & \vdots \\
T h_{m 1}(\alpha) & T h_{m 2}(\alpha) & \ldots & 1+T h_{m m}(\alpha)
\end{array}\right]
$$

Recalling that $\|\cdot\|_{\infty}$ on $\mathbb{R}^{m \times n}$ is defined as a maximum row sum of the elements of the matrix, (A.6) translates to

$$
\max _{i}\left(\left|1+T h_{i i}(\alpha)\right|+T \sum_{\substack{j=1 \\ j \neq i}}^{m}\left|h_{i j}(\alpha)\right|\right)<1
$$

which in turn means that

$$
\left|1+T h_{i i}(\alpha)\right|+T \sum_{\substack{j=1 \\ j \neq i}}^{m}\left|h_{i j}(\alpha)\right|<1 \quad \text { for all } i=1,2, \ldots, m .
$$


The above means that, in particular, $\left|1+T h_{i i}(\alpha)\right|<1$ must hold for all $i=1,2, \ldots, m$. This is true for $T \in(0, \bar{\tau})$ if and only if $h_{i i}(\alpha)<0$ for all $i=1,2, \ldots, m$. We would like to choose $T^{*} \in(0, \bar{\tau})$ such that $1+T h_{i i}(\alpha)>0$ for all $T \in\left(0, T^{*}\right)$ and $i=1,2, \ldots, m$. If we define

$$
T^{*}:=\min \left\{\frac{1}{\max _{i}\left|h_{i i}(\alpha)\right|}, \frac{\bar{\tau}}{2}\right\}
$$

then for every $T \in\left(0, T^{*}\right)$ we have that $1+T h_{i i}(\alpha)$ is positive and equation (A.7) transforms to

$$
h_{i i}(\alpha)+\sum_{\substack{j=1 \\ j \neq i}}^{m}\left|h_{i j}(\alpha)\right|<0 \quad \text { for all } i=1,2, \ldots, m .
$$

We will now prove a result that is stronger than (A.6), i.e. there exists $\lambda^{*}>0$ such that for all $\lambda \in\left(0, \lambda^{*}\right)$ and $T \in\left(0, T^{*}\right)$ we have

$$
\|I+T H(\alpha)\|_{\infty}<1-\lambda T
$$

which is equivalent to

$$
h_{i i}(\alpha)+\sum_{\substack{j=1 \\ j \neq i}}^{m}\left|h_{i j}(\alpha)\right|<-\lambda \text { for all } i=1,2, \ldots, m
$$

Having already established that the left hand side is negative, in order for (A.10) to hold we need

$$
\lambda<\left|h_{i i}(\alpha)+\sum_{\substack{j=1 \\ j \neq i}}^{m}\right| h_{i j}(\alpha)|| \quad \text { for all } i=1,2, \ldots, m
$$

Defining $\lambda^{*}$ as

$$
\lambda^{*}:=\min _{i}\left|h_{i i}(\alpha)+\sum_{\substack{j=1 \\ j \neq i}}^{m}\right| h_{i j}(\alpha)||,
$$

which is positive because of (A.8), we see that (A.9) is clearly satisfied for all $\lambda \in\left(0, \lambda^{*}\right)$ and $T \in\left(0, T^{*}\right)$.

Of course, the parameter $\alpha$ is not fixed, since it is free to vary among the compact set $\mathcal{A}$, and (3.9) must be satisfied for all $\alpha \in \mathcal{A}$. To this end, we define

$$
\bar{T}:=\min \left\{\frac{1}{\max _{\alpha} \max _{i}\left|h_{i i}(\alpha)\right|}, \frac{\bar{\tau}}{2}\right\}=\min \left\{\frac{1}{\max _{i} \max _{\alpha}\left|h_{i i}(\alpha)\right|}, \frac{\bar{\tau}}{2}\right\}
$$


and

$$
\bar{\lambda}:=\min _{\alpha} \min _{i}\left|h_{i i}(\alpha)+\sum_{\substack{j=1 \\ j \neq i}}^{m}\right| h_{i j}(\alpha)\left|=\min _{i} \min _{\alpha}\right| h_{i i}(\alpha)+\sum_{\substack{j=1 \\ j \neq i}}^{m}\left|h_{i j}(\alpha)\right| \mid .
$$

It is easy to see that $(3.9)$ is satisfied for all $T \in(0, \bar{T})$ and $\lambda \in(0, \bar{\lambda})$.

Proof of Proposition 3.

Part 1: we will first prove that if $H(\alpha) \in \mathcal{H}_{1}$, then (3.10) is LPV exponentially stable. We define a compact set

$$
\mathcal{H}:=\{H(\alpha): \alpha \in \mathcal{A}\}
$$

and let $\bar{h}:=\max _{H \in \mathcal{H}}\|H\|$. From Proposition 1 , there exist $\lambda>0$ and $\bar{T}>0$ such that for all $T \in(0, \bar{T})$ we have

$$
\|I+T H\|<1-\lambda T, \quad \text { for all } \quad H \in \mathcal{H}
$$

since $\mathcal{H}$ is compact, there exists a $\bar{\lambda}>\lambda$ so that

$$
\|I+T H\| \leq 1-\bar{\lambda} T, \quad \text { for all } H \in \mathcal{H} .
$$

With $\mathcal{H}_{c}$ being the closure of convex hull of $\mathcal{H}$, it is easy to see that for all $T \in(0, \bar{T})$ we have

$$
\|I+T H\| \leq 1-\bar{\lambda} T, \quad \text { for all } H \in \mathcal{H}_{c} .
$$

Now consider the differential equation

$$
\dot{x}(t)=H(\alpha(t)) x(t), \quad x\left(t_{0}\right)=x_{0},
$$

the solution to which is

$$
x(t)=x\left(t_{0}\right)+\int_{t_{0}}^{t} H(\alpha(\tau)) x(\tau) d \tau, \quad t \geq t_{0} .
$$

Taking the norms of both sides we have

$$
\|x(t)\| \leq\left\|x\left(t_{0}\right)\right\|+\int_{t_{0}}^{t}\|H(\alpha(\tau))\|\|x(\tau)\| d \tau
$$

Applying Bellman-Gronwall inequality to (A.12) yields

$$
\begin{aligned}
\|x(t)\| & \leq\left\|x\left(t_{0}\right)\right\| e^{\int_{t_{0}}^{t}\|H(\alpha(\tau))\| d \tau} \\
& \leq\left\|x\left(t_{0}\right)\right\| e^{\bar{h}\left(t-t_{0}\right)}
\end{aligned}
$$


which means that

$$
\begin{aligned}
\left\|x(t)-x\left(t_{0}\right)\right\| & \leq\|x(t)\|-\left\|x\left(t_{0}\right)\right\| \\
& \leq\left(e^{\bar{h}\left(t-t_{0}\right)}-1\right)\left\|x\left(t_{0}\right)\right\|, \quad t \geq t_{0} .
\end{aligned}
$$

Now let $T \in(0, \bar{T})$ and define

$$
H_{k}:=\frac{1}{T} \int_{t_{0}+k T}^{t_{0}+(k+1) T} H(\alpha(\tau)) d \tau ;
$$

using a basic property of Riemann integration it is easy to see that $H_{k} \in \mathcal{H}_{c}$. The solution of (A.11) satisfies

$$
\begin{aligned}
x\left(t_{0}+(k+1) T\right)= & x\left(t_{0}+k T\right)+\int_{t_{0}+k T}^{t_{0}+(k+1) T} H(\alpha(\tau)) x(\tau) d \tau \\
= & x\left(t_{0}+k T\right)+\int_{t_{0}+k T}^{t_{0}+(k+1) T} H(\alpha(\tau)) x\left(t_{0}+k T\right) d \tau \\
& +\underbrace{\int_{t_{0}+k T}^{t_{0}+(k+1) T} H(\alpha(\tau))\left[x(\tau)-x\left(t_{0}+k T\right)\right] d \tau}_{=: \Delta_{k}}
\end{aligned}
$$

Using (A.14) we see that

$$
\begin{aligned}
& \left\|\Delta_{k}\right\| \leq \bar{h} \int_{t_{0}+k T}^{t_{0}+(k+1) T}\left(e^{\bar{h}\left(\tau-t_{0}-k T\right)}-1\right)\left\|x\left(t_{0}+k T\right)\right\| d \tau \\
& =\bar{h}\left\|x\left(t_{0}+k T\right)\right\|\left[\frac{1}{\bar{h}}\left(e^{\bar{h} T}-1\right)-T\right]
\end{aligned}
$$

It is easy to see that there exists $T^{*} \in(0, \bar{T})$ such that $e^{\bar{h} T} \leq 1+\bar{h} T+\bar{h}^{2} T^{2}$ for $T \in\left(0, T^{*}\right)$. Hence, for small $T$ we have

$$
\begin{aligned}
\left\|\Delta_{k}\right\| & \leq \bar{h}\left\|x\left(t_{0}+k T\right)\right\|\left[\frac{1}{\bar{h}}\left(\bar{h} T+\bar{h}^{2} T^{2}\right)-T\right] \\
& =\left\|x\left(t_{0}+k T\right)\right\| \bar{h}^{2} T^{2} .
\end{aligned}
$$

Now we can calculate a bound on $\left\|x\left(t_{0}+(k+1) T\right)\right\|$ for $k \in \mathbb{Z}^{+}$:

$$
\begin{aligned}
\left\|x\left(t_{0}+(k+1) T\right)\right\| & \leq\left\|1+T H_{k}\right\|\left\|x\left(t_{0}+k T\right)\right\| d \tau+\left\|\Delta_{k}\right\| \\
& \leq\left(1-\bar{\lambda} T+\bar{h}^{2} T^{2}\right)\left\|x\left(t_{0}+k T\right)\right\| \\
& \leq\left(1-\frac{\lambda+\bar{\lambda}}{2} T\right)\left\|x\left(t_{0}+k T\right)\right\| \quad \text { if } \quad T \leq \frac{\bar{\lambda}-\lambda}{2 \bar{h}^{2}} .
\end{aligned}
$$


Therefore, letting $T^{\prime}=\min \left\{T^{*}, \frac{\bar{\lambda}-\lambda}{2 \bar{h}^{2}}\right\}$, for $T \in\left(0, T^{\prime}\right)$ we have

$$
\left\|x\left(t_{0}+k T\right)\right\| \leq\left(1-\frac{\lambda+\bar{\lambda}}{2} T\right)^{k}\left\|x\left(t_{0}\right)\right\|, \quad k \in \mathbb{Z}^{+} .
$$

Furthermore, since $\bar{\lambda}>\lambda$, we have

$$
\begin{aligned}
1-\frac{\lambda+\bar{\lambda}}{2} T & <1-\lambda T \\
& <e^{-\lambda T}
\end{aligned}
$$

Hence, (A.15) can be written as

$$
\left\|x\left(t_{0}+k T\right)\right\| \leq e^{-\lambda k T}\left\|x\left(t_{0}\right)\right\|, \quad k \in \mathbb{Z}^{+} .
$$

Now we use (A.13) to bound $\|x(t)\|$ when $t \in\left[t_{0}+k T, t_{0}+(k+1) T\right]$. For $T \in\left(0, T^{\prime}\right)$ we have

$$
\begin{aligned}
\|x(t)\| & \leq e^{\bar{h} T}\left\|x\left(t_{0}+k T\right)\right\| \\
& \leq e^{\bar{h} T} e^{-\lambda k T}\left\|x\left(t_{0}\right)\right\| \quad k \in \mathbb{Z}^{+} .
\end{aligned}
$$

But $t-t_{0} \in[k T,(k+1) T]$, which means that $e^{-\lambda\left(t-t_{0}-T\right)} \in\left[e^{-\lambda k T}, e^{-\lambda(k-1) T}\right]$. Therefore,

$$
\begin{aligned}
\|x(t)\| & \leq e^{\bar{h} T} e^{-\lambda\left(t-t_{0}-T\right)}\left\|x\left(t_{0}\right)\right\| \\
& \leq e^{\bar{h} T} e^{\lambda T} e^{-\lambda\left(t-t_{0}\right)}\left\|x\left(t_{0}\right)\right\|, \quad t \geq t_{0} .
\end{aligned}
$$

Since the above is true for all $T \in\left(0, T^{\prime}\right)$, it means that

$$
\|x(t)\| \leq e^{-\lambda\left(t-t_{0}\right)}\left\|x\left(t_{0}\right)\right\|, \quad t \geq t_{0} .
$$

This concludes the proof of Part 1.

Part 2: we will now prove that if $H(\alpha) \in \mathcal{H}_{\infty}$, then (3.10) is LPV exponentially stable. The proof is identical to Part 1 with the only exception that $\|\cdot\|_{\infty}$ is used in place of $\|\cdot\|$. Hence, we have

$$
\|x(t)\|_{\infty} \leq e^{-\lambda\left(t-t_{0}\right)}\left\|x\left(t_{0}\right)\right\|_{\infty}, \quad t \geq t_{0} .
$$

By norm equivalence on $\mathbb{R}^{n}$, there exists $\gamma>0$ such that

$$
\|x(t)\| \leq \gamma e^{-\lambda\left(t-t_{0}\right)}\left\|x\left(t_{0}\right)\right\|, \quad t \geq t_{0} .
$$


Proof of Lemma 1. Integrating (3.26) we obtain

$$
x(t)=x\left(t_{0}\right)+\int_{t_{0}}^{t} A(\alpha(\tau)) x(\tau) \mathrm{d} \tau+\int_{t_{0}}^{t} B(\alpha(\tau)) u(\tau) \mathrm{d} \tau
$$

Now we take a norm of both sides and apply the triangle inequality

$$
\begin{aligned}
\|x(t)\| & \leq\left\|x\left(t_{0}\right)\right\|+\left\|\int_{t_{0}}^{t} A(\alpha(\tau)) x(\tau) \mathrm{d} \tau\right\|+\left\|\int_{t_{0}}^{t} B(\alpha(\tau)) u(\tau) \mathrm{d} \tau\right\| \\
& \leq\left\|x\left(t_{0}\right)\right\|+\int_{t_{0}}^{t}\|A(\alpha(\tau)) x(\tau)\| \mathrm{d} \tau+\int_{t_{0}}^{t}\|B(\alpha(\tau)) u(\tau)\| \mathrm{d} \tau \\
& \leq\left\|x\left(t_{0}\right)\right\|+\int_{t_{0}}^{t}\|A(\alpha(\tau))\|\|x(\tau)\| \mathrm{d} \tau+\int_{t_{0}}^{t}\|B(\alpha(\tau))\|\|u(\tau)\| \mathrm{d} \tau
\end{aligned}
$$

Now we can apply bounds from (3.28) and (4.9)

$$
\|x(t)\| \leq\left\|x\left(t_{0}\right)\right\|+\left(t-t_{0}\right) c_{b} \bar{u}+\int_{t_{0}}^{t} c_{a}\|x(\tau)\| \mathrm{d} \tau
$$

Since $\left\|x\left(t_{0}\right)\right\|+\left(t-t_{0}\right) c_{b} \bar{u} \geq 0$ we can apply the Bellman-Gronwall inequality to obtain

$$
\begin{aligned}
\|x(t)\| & \leq\left[\left\|x\left(t_{0}\right)\right\|+\left(t-t_{0}\right) c_{b} \bar{u}\right] e^{\int_{t_{0}}^{t} c_{a} \mathrm{~d} \tau} \\
& =\left[\left\|x\left(t_{0}\right)\right\|+\left(t-t_{0}\right) c_{b} \bar{u}\right] e^{c_{a}\left(t-t_{0}\right)}
\end{aligned}
$$

This expression is identical to (4.10). 


\section{References}

[1] A.M. Annaswamy and K.S. Narendra. Adaptive control of a first order plant with a time-varying parameter. American Control Conference, pages 975-980, June 1989.

[2] F. Blanchini. Ultimate boundedness control for uncertain discrete-time systems via set-induced lyapunov functions. Proceeding of the 30th IEEE Conference on Decision and Control, (2):1755-1760, December 1991.

[3] F. Blanchini. Nonquadratic lyapunov functions for robust control. Automatica, 31(3):451-461, March 1995.

[4] F. Blanchini. The gain scheduling and the robust state feedback stabilization problems. IEEE Trans. Autom. Control, 45(11):2061-2070, November 2000.

[5] F. Blanchini, D. Casagrande, S. Miani, and U. Viaro. Stable LPV realization of parametric transfer functions and its application to gain-scheduling control design. IEEE Trans. Autom. Control, 55(10):2271-2281, October 2010.

[6] F. Blanchini, S. Miani, and C. Savorgnan. Stability results for linear parameter varying and switching systems. Automatica, 43(10):1817-1823, October 2007.

[7] C.I. Byrnes and J.C. Willems. Adaptive stabilization of multivariable linear systems. The 23rd IEEE Conference on Decision and Control, 23:1574-1577, December 1984.

[8] D. Dimogianopoulos and R. Lozano. Adaptive control for linear slowly time-varying systems using direct least-squares estimation. Automatica, 37(2):251-2561, February 2001.

[9] B. Fidan, Y. Zhang, and P. Ioannou. Adaptive control of a class of slowly time-varying systems with modelling uncertainties. IEEE Trans. Autom. Control, 50(6):915-920, June 2005. 
[10] G.C. Goodwin, P.J. Ramadge, and P.E. Caines. Discrete-time multivariable adaptive control. IEEE Trans. Autom. Control, 25(3):449-456, June 1980.

[11] G.C. Goodwin and K.S. Sin. Adaptive Filtering Prediction and Control. Prentice-Hall, 1984.

[12] C.M. Hackl, N. Hopfe, A. Ilchmann, M. Mueller, and S. Trenn. Funnel control for systems with relative degree two. SIAM Journal of Control and Optimization, 51(2):965995, 2013.

[13] J.P. Hespanha, D. Liberzon, and A.S. Morse. Overcoming the limitations of adaptive control by means of logic based switching. Systems and Control Letters, 49(1):49-65, May 2003.

[14] J. Hocherman-Frommer, S. R. Kulkarni, and P. J. Ramadge. Controller switching based on output prediction errors. IEEE Trans. Autom. Control, 43(5):596-607, May 1998.

[15] A. Ilchmann, E.P. Ryan, and S. Trenn. Tracking control: Performance funnels and prescribed transient behaviour. Systems and Control Letters, 54(7):655-670, July 2005.

[16] P. A. Ioannou and J. Sun. Robust Adaptive Control. Prentice Hall, 1996.

[17] G. Kreisselmeir. Adaptive control of a class of slowly time-varying plants. Systems and Control Letters, 8(2):97-103, December 1986.

[18] R. Marino and P. Tomei. Adaptive control of linear time-varying systems. Automatica, 39(4):651-659, April 2003.

[19] R.H. Middleton and G.C. Goodwin. Adaptive control of time-varying linear systems. IEEE Trans. Autom. Control, 33(2):150-155, February 1988.

[20] D.E. Miller. A new approach to model reference adaptive control. IEEE Trans. Autom. Control, 48(5):743-757, May 2003.

[21] D.E. Miller. Near optimal LQR performance for a compact set of plants. IEEE Trans. Autom. Control, 51(9):1423-1439, September 2006.

[22] D.E. Miller and E.J. Davison. An adaptive controller which provides an arbitrarily good transient and steady-state response. IEEE Trans. Autom. Control, 36(1):68-81, January 1991. 
[23] D.E. Miller and N. Mansouri. Model reference adaptive control using simultaneous probing, estimation, and control. IEEE Trans. Autom. Control, 55(9):2014-2029, September 2010.

[24] D.E. Miller and J.R. Vale. Pole placement adaptive control with persistent jumps in the plant parameters. Mathematics of Control, Signals, and Systems, in press.

[25] A.S. Morse. Global stability of parameter-adaptive control systems. IEEE Trans. Autom. Control, 25(3):433-439, June 1980.

[26] A.S. Morse. Supervisory control of families of linear set-point controllers - part 1: Exact matching. IEEE Trans. Autom. Control, 41(10):1413-1431, October 1996.

[27] A.S. Morse. Supervisory control of families of linear set-point controllers - part 2: Robustness. IEEE Trans. Autom. Control, 42(11):1500-1515, November 1997.

[28] K.S. Narendra and J. Balakrishnan. Adaptive control using multiple models. IEEE Trans. Autom. Control, 42(2):171-187, February 1997.

[29] K.S. Narendra, Y.H. Lin, and L.S. Valavani. Stable adaptive controller design, part II: Proof of stability. IEEE Trans. Autom. Control, 25(3):440-448, June 1980.

[30] C. E. Rohrs, L. Valavani, M. Athans, and G. Stein. Robustness of adaptive control algorithms in the presence of unmodeled dynamics. IEEE Trans. Autom. Control, 30(9):881-889, September 1985.

[31] W.J. Rugh. Analytical framework for gain scheduling. IEEE Control Systems, 11(1):79-84, January 1991.

[32] W.J. Rugh and J.S. Shamma. Research on gain scheduling. Automatica, 36(10):14011425, October 2000.

[33] J. Shamma. An overview of LPV systems. In J. Mohammadpour and C. Scherer, editors, Control of Linear Parameter Varying Systems with Applications, pages 3-26. Springer, 2012.

[34] J.S. Shamma and M. Athans. Analysis of gain scheduled control for nonlinear plants. IEEE Trans. Autom. Control, 35(8):898-907, August 1990.

[35] Z. Tian and K.S. Narendra. Adaptive control of linear periodic systems. 2009 American Control Conference, June 2009. 
[36] K.S. Tsakalis and P.A. Ioannou. Adaptive control of linear time-varying plants: A new model reference controller structure. IEEE Trans. Autom. Control, 34(10):1038-1046, October 1989.

[37] K.S. Tsakalis and P.A. Ioannou. A new indirect adaptive control scheme for timevarying plants. IEEE Trans. Autom. Control, 35(6):697-705, June 1990.

[38] J.R. Vale and D.E. Miller. Step tracking in the presence of persistent plant changes. IEEE Trans. Autom. Control, 56(1):43-58, January 2011.

[39] P.G. Voulgaris, M.A. Dahleh, and L.S. Valavani. Robust adaptive control: a slowly varying systems approach. Automatica, 30(9):1455-1461, September 1994.

[40] L. Vu and D. Liberzon. Supervisory control of uncertain linear time-varying systems. IEEE Trans. Autom. Control, 56(1):27-42, January 2011.

[41] Fuzhen Zhang. Matrix theory: basic results and techniques. Springer-Verlag New York Inc, 1999. 